\title{
MULTICHANNEL SPECTRUM SENSING OVER CORRELATED FADING CHANNELS WITH DIVERSITY RECEPTION
}

\author{
by \\ Salam Al-Juboori \\ B.Sc, University of Baghdad, Baghdad, Iraq, 1982 \\ M.Sc, University of Technology, Baghdad, Iraq, 2008 \\ M.Eng, McMaster University, Hamilton, Ontario, Canada, 2011 \\ A Dissertation \\ presented to Ryerson University \\ in partial fulfillment of the requirements for the degree of \\ Doctor of Philosophy \\ in the Program of \\ Electrical and Computer Engineering \\ Toronto, Ontario, Canada, 2017 \\ (C)Salam Al-Juboori, 2017
}




\section{AUTHORS DECLARATION FOR ELECTRONIC SUBMISSION OF A DISSERTATION}

I hereby declare that I am the sole author of this dissertation. This is a true copy of the dissertation, including any required final revisions, as accepted by my examiners.

I authorize Ryerson University to lend this dissertation to other institutions or individuals for the purpose of scholarly research.

I further authorize Ryerson University to reproduce this dissertation by photocopying or by other means, in total or in part, at the request of other institutions or individuals for the purpose of scholarly research.

I understand that my dissertation may be made electronically available to the public. 


\title{
Abstract \\ MULTICHANNEL SPECTRUM SENSING OVER CORRELATED FADING CHANNELS WITH DIVERSITY RECEPTION
}

\author{
(C)Salam Al-Juboori, 2017 Doctor of Philosophy \\ Electrical and Computer Engineering \\ Ryerson University
}

Accurate detection of white spaces is crucial to protect primary user against interference with secondary user. Multipath fading and correlation among diversity branches represent essential challenges in Cognitive Radio Network Spectrum Sensing (CRNSS). This dissertation investigates the problem of correlation among multiple diversity receivers in wireless communications in the presence of multipath fading. The work of this dissertation falls into two folds, analysis and solution. In the analysis fold, this dissertation implements a unified approach of performance analysis for cognitive spectrum sensing. It considers a more realistic sensing scenario where non-independent multipath fading channels with diversity combining technique are assumed. Maximum Ratio Combining (MRC), Equal Gain Combining (EGC), Selection Combining (SC) and Selection and Stay Combining (SSC) techniques are employed. Arbitrarily, constant and exponentially dual, triple and $L$ number of Nakagami- $m$ correlated fading branches are investigated. We derive novel closed-form expressions for the average detection probability for each sensing scenario with simpler and more general alternative expressions. Our numerical analysis reveals the deterioration in detection probability due to correlation especially in deep fading. Consequently, an increase in the interference rate between the primary user and secondary user is observed by three 
times its rate when independent fading branches is assumed. However, results also show that this effect could be compensated for, through employing the appropriate diversity technique and by increasing the diversity branches. Therefore, we say that the correlation cannot be overlooked in deep fading, however in low fading can be ignored so as to reduce complexity and computation. Furthermore, at low fading, low false alarm probability and highly correlated environments, EGC which is simpler scheme performs as good as MRC which is a more complex scheme. Similar result are observed for SC and SSC. For the solution fold and towards combatting the correlation impact on the wireless systems, a decorrelator implementation at the receiver will be very beneficial. We propose such decorrelator scheme which would significantly alleviate the correlation effect. We derive closed-form expressions for the decorrelator receiver detection statistics including the Probability Density Function (PDF) from fundamental principles, considering dual antenna SC receiver in Nakagami- $m$ fading channels. Numerical results show that the PDF of the bivariate difference could be perfectly represented by a semi-standard normal distribution with zero mean and constant variance depending on the bivariate's parameters. This observation would significantly help simplifying the design of decorrelator receiver. The derived statistics can be used in the problem of self-interference for multicarrier systems. Results also show the outage probability has been improved by double, due to the decorrelator. 


\section{Acknowledgment}

All praises are due to Almightily Allah, the most Gracious, and the most Merciful. I thank Him for all the bounties and blessings He bestowed upon me and for granting me the strength and patience to complete this work.

I would like to express my sincerest gratitude to my supervisor, Professor Xavier Fernando, for his patient guidance, warm encouragement and invaluable advice throughout the course of my PhD study. He was always understanding of the difficulties and the challenges one inevitably faces during the seemingly never ending PhD study. His positive outlook and endless support inspired me and gave me the confidence and motivation needed to finish this work. His careful and insightful reviews contributed enormously to the production of this thesis. It was truly a great privilege working with him as I learnt from him a lot that will pave the road in my future.

I would like to thank my thesis committee members, chairs and professors, Professor Olivia Das, Professor Songnian Li, Professor Lian Zhao, Professor Krishna D. Kumar and Professor Isaac Woungang for taking the time and effort to review my thesis and provide me with their insightful and valuable comments. My deep appreciations to Professor Chintha Tellambura, External Examiner, University of Alberta, for accepting to be my external examiner and provide me with his valuable comments. I would also like to acknowledge the administrative staff at the school of graduate studies and the department of Electrical and Computer Engineering at Ryerson University for all the instances in which their assistance helped me along the way. I would especially like to thank Dr. Atef T. for his help in some mathematical problems derivation. Finally, I would like to thank my colleagues at Ryerson Communication Lab (RLC) and research group present and past, I am lucky to be a part of this group where a 
team spirit truly prevails. Our group discussions and their insightful feedback have helped in many occasions. 


\section{Table of Contents}

Declaration ................... ii

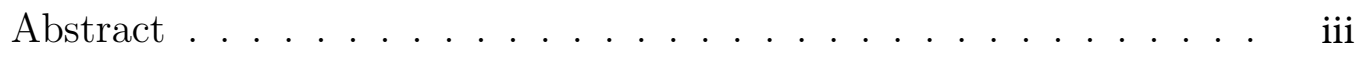

Acknowledgment ................... iv

List of Tables . . . . . . . . . . . . . . . . xi

List of Figures . . . . . . . . . . . . . . . xiv

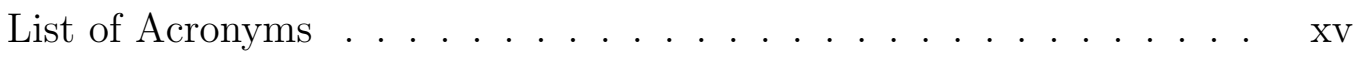

List of Symbols . . . . . . . . . . . . . . . . . xvii

1 Introduction 1

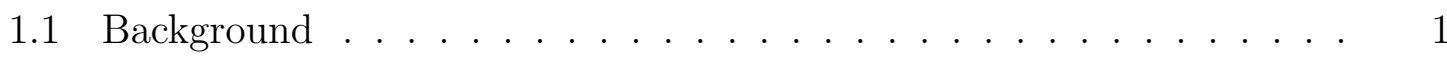

1.2 Cognitive Radio Spectrum Sensing (CRSS)

Techniques . . . . . . . . . . . . . . . . . 3

1.3 Spectrum Sensing Challenges $\ldots \ldots \ldots \ldots \ldots \ldots$

1.4 Motivation ........................ 7

1.5 Related Work . . . . . . . . . . . . . . . . . . 8

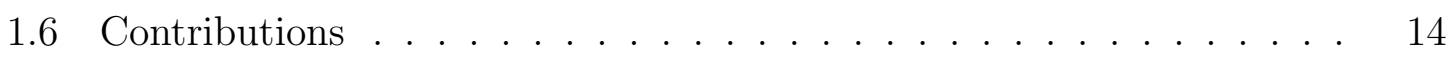

1.7 Thesis Outline . . . . . . . . . . . . . . . . . . . . . 17

2 Spectrum Sensing in Correlated Multipath Fading Channels 20 
2.1 Signal Detection Techniques . . . . . . . . . . . . . . . . . 21

2.1 .1 Energy Detection . . . . . . . . . . . . . . . . 21

2.1 .2 Matched Filter . . . . . . . . . . . . . . . . . . 23

2.1.3 Cyclostationary Feature . . . . . . . . . . . . . . . 24

2.2 Wireless Channel . . . . . . . . . . . . . . . . . 24

2.2.1 Free-Space Model . . . . . . . . . . . . . . . . . 25

2.2.2 Multipath Fading Model . . . . . . . . . . . 25

2.3 Spectrum Sensing with Diversity Combining . . . . . . . . . . . 27

2.4 Correlation in Wireless Channels . . . . . . . . . . . . . . 30

2.5 Signal Detection in Correlated Multipath

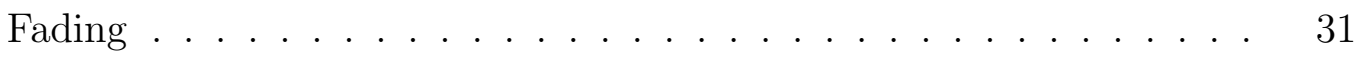

2.5.1 System Model . . . . . . . . . . . . . . . . . . . . . 32

2.6 Problem Formulation . . . . . . . . . . . . . . . . . . 35

$3 \quad L$ Correlated Nakagami- $m$ Branches with MRC Diversity 37

3.1 MRC Diversity . . . . . . . . . . . . . . . . . . . . 38

3.2 Constant Correlation . . . . . . . . . . . . . . . . . . . . . 41

3.2.1 Alternative Expression for ${\overline{P_{D}}}_{M C \text { :Const }} \ldots \ldots$. . . . . . . 44

3.3 Exponential Correlation . . . . . . . . . . . . . . . . . . 46

3.3.1 Alternative $\bar{P}_{D: M R C: E x p}$ Simple Expression . . . . . . . . 49

3.3.2 Arbitrary Real $u$-values Alternative

$\bar{P}_{D: M R C: E x p}$ Expression $\ldots \ldots \ldots \ldots$

3.4 Performance Analysis . . . . . . . . . . . . . . . . . . . . . 51

4 Dual Correlated Nakagami- $m$ Branches with EGC Diversity $\quad 57$

4.1 EGC Diversity . . . . . . . . . . . . . . . . . 57 
$4.2 \bar{P}_{D: E G C, 2}$ Expression $\ldots \ldots \ldots \ldots \ldots \ldots \ldots \ldots$

4.3 Alternative $\bar{P}_{D: E G C, 2}$ Expression $\ldots \ldots \ldots \ldots \ldots$

4.4 Performance Analysis . . . . . . . . . . . . . . . . . . . . . . . 61

5 Correlated Nakagami- $m$ Branches with SC Diversity 65

5.1 SC Diversity . . . . . . . . . . . . . . . . . . . . . 66

5.2 Dual Arbitrarily Correlated Branches . . . . . . . . . . . . . 67

5.2 .1 Average Detection Probability . . . . . . . . . . . 68

$5.2 .2 \quad \bar{P}_{D, S C, 2}$ Alternative Expression $\ldots \ldots \ldots \ldots \ldots$

5.3 Triple Exponentially Correlated SC . . . . . . . . . . . 76

5.3.1 Average Detection Probability, $\bar{P}_{D, S C, 3} \ldots \ldots \ldots \ldots$

5.3.2 Alternative $\bar{P}_{D, S C, 3}$ Expression $\ldots \ldots \ldots \ldots \ldots$

5.4 Performance Analysis . . . . . . . . . . . . . . . . . . . . 81

6 Dual Correlated Nakagami- $m$ Branches with SSC Diversity 84

6.1 SSC Diversity . . . . . . . . . . . . . . . . . . . . . 84

6.2 Dual Arbitrarily Correlated Branches . . . . . . . . . . . . . 86

6.2 .1 Case: $\gamma_{T}=0 \ldots \ldots \ldots \ldots \ldots \ldots \ldots$

6.2 .2 Case: $\gamma_{T} \rightarrow \infty \ldots \ldots \ldots \ldots \ldots \ldots$. . . . . . . . 87

6.2 .3 Integral $I_{A}$ in $(6.3) \ldots \ldots \ldots \ldots \ldots$

6.2 .4 Integral $I_{B}$ in $(6.3) \ldots \ldots \ldots \ldots \ldots \ldots$

6.2 .5 Integral $I_{C}$ in $(6.3) \ldots \ldots \ldots \ldots \ldots$

6.3 Alternative $\bar{P}_{D, S S C, 2}$ Expression $\ldots \ldots \ldots \ldots \ldots$

6.3 .1 Evaluation the integral $I_{A}$ in $(6.3) \ldots \ldots \ldots \ldots 1$

6.3 .2 Evaluation the integral $I_{B}$ in $(6.3) \ldots \ldots \ldots 9 . \ldots$

6.3.3 Evaluation the integral $I_{C}$ in $(6.3) \ldots \ldots \ldots \ldots$ 
6.4 Optimum Threshold $\left(\gamma_{T}^{*}\right) \ldots \ldots \ldots$. . . . . . . . . . 93

6.5 Performance Evaluation _. . . . . . . . . . . . . . . . . 96

7 Decorrelation-Based SC Receiver in Nakagami-m Fading Channel 99

7.1 System Model . . . . . . . . . . . . . . . . . . . . . . . 102

7.2 Statistics of Normalized Difference of Identical and correlated Nakagami- $m$

Bivariate: $\delta=\frac{r_{1}-r_{2}}{\sqrt{2}} \ldots \ldots \ldots \ldots 10 \ldots \ldots$

7.2.1 PDF's Expression: $f_{\Delta}(\delta) \ldots \ldots \ldots \ldots$. . . . . . . . 104

7.2.2 Alternative PDF's Expression . . . . . . . . . . . . . . 108

7.2.3 Cumulative Distribution Function (CDF) Expression . . . . . 110

7.3 The CDF of the Decorrelator . . . . . . . . . . . . . . . 112

7.4 Outage Probability . . . . . . . . . . . . . . . . . 114

7.5 Numerical Analysis . . . . . . . . . . . . . . . . . . . . . . 115

8 Comparison of Different Schemes $\quad 121$

$8.1 \mathrm{MRC} / \mathrm{EGC}$ Comparison . . . . . . . . . . . . . . . . 121

$8.2 \mathrm{SC} / \mathrm{SSC}$ Comparison . . . . . . . . . . . . . . . 123

8.3 Comprehensive Comparison . . . . . . . . . . . . . . . 127

8.4 Decorrelator Performance . . . . . . . . . . . . . . . . . 130

9 Conclusion and Future Work 131

9.1 Conclusion . . . . . . . . . . . . . . . . . . . . . . 131

9.2 Future Work . . . . . . . . . . . . . . . . . . . . . . 134

$\begin{array}{lr}\text { Bibliography } & 136\end{array}$ 


\section{List of Tables}

3.1 Terms required for five digits accuracy . . . . . . . . . . . . 50

4.1 Terms required for five digits accuracy . . . . . . . . . . . . . 61

5.1 Terms required for five digits accuracy . . . . . . . . . . . . . 81

7.1 Terms required for five digits accuracy . . . . . . . . . . . . . 113 


\section{List of Figures}

1.1 Cognitive radio spectrum sensing cycle. . . . . . . . . . . . . . 2

1.2 Cognitive radio components. . . . . . . . . . . . . . . . 3

1.3 Few Cognitive radio spectrum sensing techniques. . . . . . . . . . . . 4

1.4 Hidden terminal problem in Cognitive Radio Spectrum Sensing. . . . 5

1.5 Summary of thesis contributions. . . . . . . . . . . . . . 15

2.1 Energy detection spectrum sensing technique. . . . . . . . . . . . 21

2.2 Matched filter detector block diagram. . . . . . . . . . . . . 23

2.3 Fading in wireless communication channel. . . . . . . . . . . . . 26

2.4 Single-Input-Multiple-Output (SIMO) system. . . . . . . . . . . . 28

3.1 Maximal Ratio Combining Technique (MRC). . . . . . . . . . . . . . 39

3.2 Analytic (solid) versus simulation (dashed) results for MRC with $L=2$,

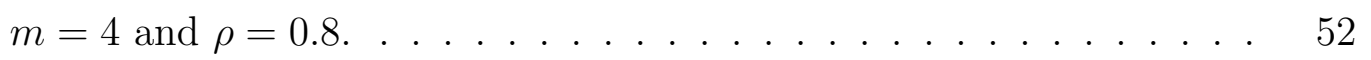

3.3 Constant correlated Nakagami- $m$ branches with MRC diversity; $\bar{\gamma}=20$ $\mathrm{dB}, L=2,3$ and 4 for different $\rho$ values. . . . . . . . . . . . 53

3.4 Exponentially correlated Nakagami- $m$ branches with MRC diversity; $\bar{\gamma}=20 \mathrm{~dB}, L=2,3$ and 4 for different $\rho$ values. . . . . . . . . . . . 54 
3.5 Correlation impact on probability of false alarm in correlated Nakagami- $m$ branches with MRC diversity; $\bar{\gamma}=20 \mathrm{~dB}, L=2,3$ and 4 for different $\rho$ values, (a) and (b) represent the exact points while in (c) and (d), corresponding points are connected with straight lines for better visualization of the performance difference. Constant correlation (solid), exponential correlation (dashed). . . . . . . . . . . 56

4.1 Equal Gain Combining (EGC). . . . . . . . . . . . . . . 58

4.2 Analytic (solid) versus simulation (dashed) results for EGC with $L=2$, $m=4$ and $\rho=0.8 \ldots \ldots \ldots \ldots \ldots$. . . . . . . . . . . . . 62

4.3 Arbitrarily correlated Nakagami- $m$ branches with EGC diversity: $L=$ $2, \bar{\gamma}=20 \mathrm{~dB}$ for different $\rho$ values. . . . . . . . . . . . . . . 63

5.1 Selection Combining Technique (SC) . . . . . . . . . . . . . . 66

5.2 Analytic (solid) versus simulation (dashed) results for SC with $L=$ $2, m=1, \bar{\gamma}=20 \mathrm{~dB}$ and $\rho=0.8 \ldots \ldots \ldots . \ldots . \ldots . \ldots . \ldots 2$

5.3 SC dual correlated Nakagami- $m$ branches with $\bar{\gamma}=20 \mathrm{~dB}$ for different $\rho$ values. . . . . . . . . . . . . . . . . . . .

6.1 Switched and Stay Combining Technique (SSC). . . . . . . . . . . . . 85

6.2 Analytic (solid) versus simulation (dashed) results for SSC with $L=$ $2, m=1, \bar{\gamma}=20 \mathrm{~dB}$ and $\rho=0.8 \ldots \ldots \ldots 6$

6.3 Dual correlated Nakagami- $m$ branches with SSC diversity; $\bar{\gamma}=20 \mathrm{~dB}$, $L=2$ and for different $\rho$ values. . . . . . . . . . . . . . . . . 98

7.1 Multiple-Input-Multiple-Output (MIMO) system. . . . . . . . . . . . 100 
7.2 The PDF of the normalized difference of correlated Nakagami- $m$ bivariate, analytical versus simulation for $m=1$ and $\Omega=1$ and $\rho=0.3$. . . . 114

7.3 The PDF of normalized difference of correlated Nakagami- $m$ bivariate for $m=1, \rho=0.3,0.6$ and $\Omega \in(1-5) \ldots \ldots \ldots 116$

7.4 The PDF of normalized difference of correlated Nakagami- $m$ bivariate for $\Omega=1, \rho=0.3,0.6$ and $m \in(1-4) \ldots \ldots \ldots 118$

7.5 The PDF of the normalized difference of i.i.d. Nakagami- $m$ bivariate, analytical (flat) versus simulation (with marker) for $m \in(1-4)$ and

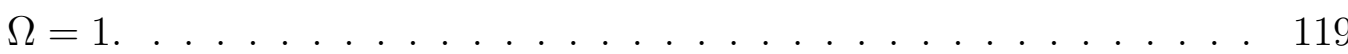

7.6 Outage probability comparison between correlated (dashed) and decorrelated (solid) SC diversity receiver in Nakagami- $m$ channel for $\bar{\gamma}=0$ dB. . . 120

8.1 Comparison of correlated Nakagami- $m$ branches with both MRC and EGC diversity techniques for $\bar{\gamma}=20 \mathrm{~dB}, L=2$ with $\rho=0$ (solid) and 0.8 (dashed). . . . . . . . . . . . . . . . . 122

8.2 SC and SSC dual correlated Nakagami- $m$ branches comparison with $\bar{\gamma}=20 \mathrm{~dB}$ for $\rho=0$ (solid) and 0.8 (dashed) . . . . . . . . . 124

8.3 Probability of miss detection versus correlation with $\bar{\gamma}=20 \mathrm{~dB}$ and different fading severity for SC and SSC . . . . . . . . . 126

8.4 MRC, EGC, SC and SSC correlated Nakagami- $m$ branches comparison for $L=2, \bar{\gamma}=20 \mathrm{~dB}$ and $\rho=0$ (solid) and 0.8 (dashed) . . . . . . 128

8.5 MRC, EGC, SC, and SSC correlated Nakagami- $m$ branches comparison for $L=2, \quad \bar{\gamma}=20 \mathrm{~dB}$ and $\rho=0$ (solid) and 0.8 (dashed). . . . . . 129 


\title{
List of Acronyms
}

\author{
AWGN Additive White Gaussian Noise \\ BER Bit Error Rate \\ CDF Cumulative Distribution Function \\ CLT Central Limit Theorem \\ CRN Cognitive Radio Network \\ CRNSS Cognitive Radio Network Spectrum Sensing \\ CRS Cognitive Radio Sensor \\ CSI Channel State Information \\ CROC Complementary Receiver Operating Characteristics \\ ED Energy Detector \\ EGC Equal Gain Combining \\ FCC Federal Communications Commission \\ GLR Generalized Likelihood Ratio \\ i.i.d. independent and identically distributed \\ i.n.i.d. independent but not identically distributed \\ n.i.i.d. non-independent and identically distributed \\ KLT Karhunen-Loeve Transformation \\ LOS Line-of-Sight
}


MGF Moment Generating Function

MIMO Multiple-Input-Multiple-Output

MG Mixture Gamma Distribution

MRC Maximum Ratio Combining

OFDM Orthogonal Frequency-Division Multiplexing

PDF Probability Density Function

PSD Power Spectral Density

SC Selection Combining

SER Symbol Error Rate

SIMO Single-Input-Multiple-Output

SINR Signal-to-Interference-Noise-Ratio

SLC Square-Law Combining

SLS Square-Law Selection Combining

SNR Signal-to-Noise-Ratio

SSC Selection and Stay Combining

TW Time Bandwidth Product 


\title{
List of Symbols
}

\author{
$\lambda$ Threshold \\ $x(t)$ Received Signal \\ $n(t)$ Noise \\ $h$ Channel Amplitude Coefficient \\ $P_{F}$ False Alarm Probability \\ $P_{D}$ Detection Probability \\ $\bar{P}_{D, \text { Div }}$ Average Detection Probability \\ $P_{D m}$ Miss Detection Probability \\ $\Gamma($.$) Gamma Function$ \\ $\Gamma(.,$.$) Incomplete Gamma Function$ \\ $Q_{u}(.,.) u^{t h}$ Order of Generalized Marcum Q-function \\ $\gamma$ Signal-to-Noise-Ratio \\ $\gamma_{T}^{*}$ Optimum Threshold \\ $\bar{\gamma}$ Average Signal-to-Noise-Ratio \\ $\sigma_{n}^{2}$ Noise Variance \\ $\sigma_{s}^{2}$ Primary User Signal Variance \\ $m$ Nakagami fading parameter \\ $\rho$ Correlation
}


$H_{0}$ Null Hypothesis

$H_{1}$ Alternative Hypothesis

$I_{v}(.) \nu$ th-order of Modified Bessel Function of First Kind

${ }_{1} F_{1}(., . ;$.$) Confluent Hypergeometric Function$

${ }_{2} F_{1}\left(a_{2} ; b_{2} ; c_{2} ; z\right)$ Gaussian Hypergeometric function

$F_{2}(. ; ., . ; ., . ; .,$.$) Hypergeometric Function of Two Variables$

$M_{\mu, \nu}($.$) Whittaker Function$

$U(. ; . ;$.$) Confluent Hypergeometric Function of the Second Kind$

W Complex Gaussian Noise Vector

H Channel Gain Coefficients Matrix

I Identity Matrix

S Primary Users Signals Matrix

X Received Observations Matrix

R Observations Covariance Matrix

(.) ${ }^{H}$ Hermitian Complex Conjugate

$\Sigma$ Population Covariance Matrix

$\left(\begin{array}{l}\beta \\ l\end{array}\right)$ Binomial Coefficient

$B(.,$.$) Beta Function$

$N \sim\left(0, \sigma^{2}\right)$ Normal Distribution 
(.) ${ }^{T}$ Transpose

$(x)_{n}$ Pochhammer Symbol

$E$ (.) Expected or Mean Value of a Random Variable

$\delta($.$) Dirac Delta Function$ 


\section{Chapter 1}

\section{Introduction}

\subsection{Background}

The radio frequency spectrum is a precious resource to enable communications among wireless devices. This resource is increasingly facing a big challenge through the huge demand on allocating frequency bands for communication devices. For example, the current Federal Communications Commission (FCC) regulations adopt a static spectrum allocation policy to the licensed users on a long-term basis and over large geographical areas. However, recent studies revealed that less than $20 \%$ of the allocated spectrum is actually utilized[1-6]. An effective solution to address this huge demand must be proposed; consequently, the static allocation policy must be changed to take advantage of the under-utilized spectrum.

A Cognitive Radio Network (CRN) approach is considered as a promising solution for solving the radio spectrum scarcity resulting from the unprecedented widen growth. J. 


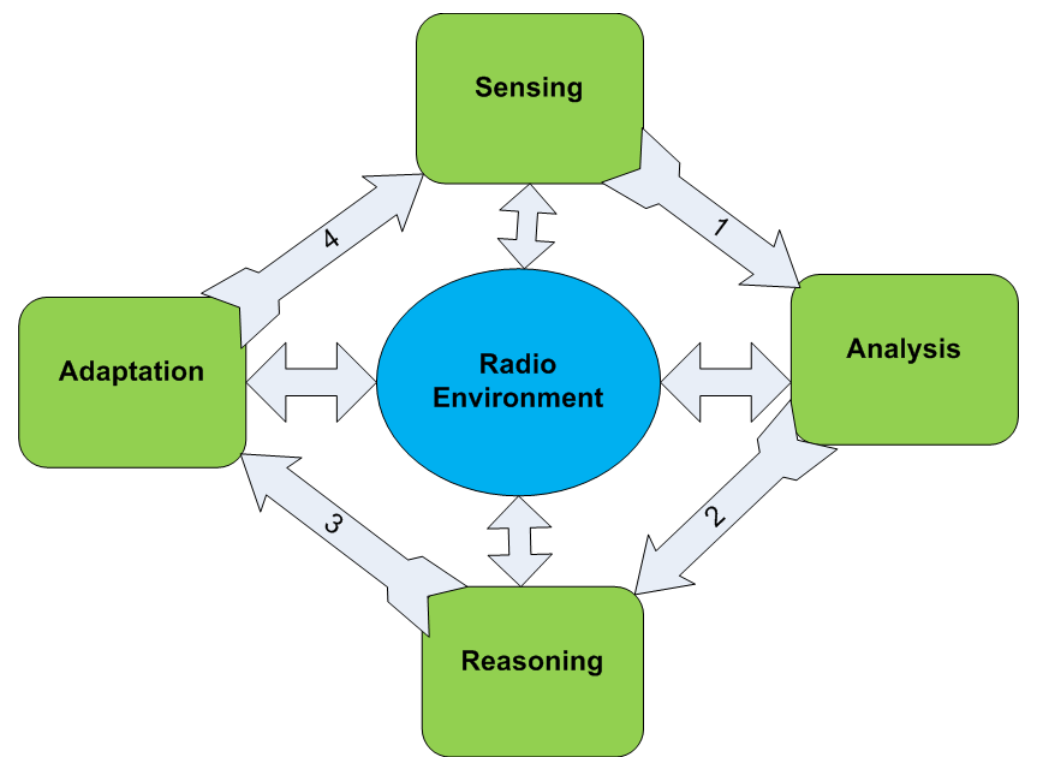

Figure 1.1: Cognitive radio spectrum sensing cycle.

Mitola [7] first proposed the concept of CRN where, a communication device is aware of its surrounding radio environment and has the ability to adjust its parameters such as: transmit power, carrier frequency and modulation strategy. The CRN device adapts these adjustable parameters in real time in order to achieve two objectives:

1. Reliable communication

2. Efficient utilization of the radio spectrum.

Since CRN devices must co-exist with licensed primary users, dynamically sensing the radio spectrum to decide the presence or absence of the primary users is a very important function of a CRN device. Accurate sensing will ensure no interference on the primary users from the secondary users who use the same frequency band while ensuring high throughput to the secondary users [8],[9] which is done through so-called cognitive cycle (Figure. 1.1) and its components (Figure. 1.2). 


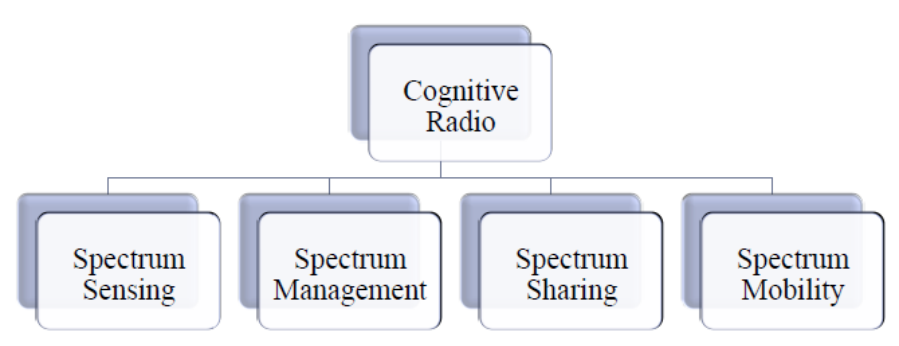

Figure 1.2: Cognitive radio components.

\subsection{Cognitive Radio Spectrum Sensing (CRSS) Techniques}

In general, spectrum sensing could be divided into three main categories (Figure. 1.3):

1. Transmitter detection

Detecting if the primary signal transmitter is locally present in a certain spectrum band or not.

2. Cooperative detection

Information from multiple sensors are combined together to detect the presence of the primary user.

3. Interference based detection

The cognitive node coexists with primary user and is allowed to transmit with low power but is restricted by the interference temperature level in order not to cause harmful interference to primary user.

In fact, the core of cognitive spectrum sensing is detecting a signal in noisy channel which is traditionally referred to as signal detection theory. There are several methods 


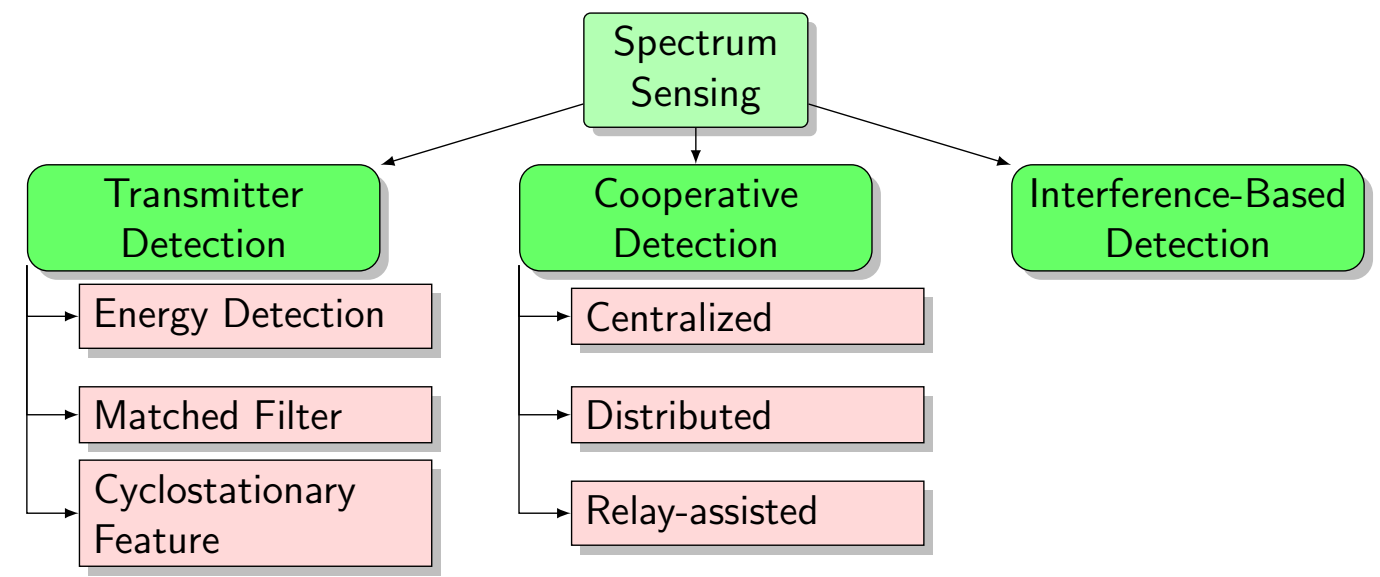

Figure 1.3: Few Cognitive radio spectrum sensing techniques.

of spectrum sensing; signal detection, based on the type of available sensing parameters information, such as frequency, modulation, signal and noise variances, collected about the primary user [10]. However, the most important aspect of the primary user signal detection is the binary hypothesis test in order to declare the presence or the absence of the primary user signal.

Different spectrum sensing techniques have been proposed depending on different available sensing parameters information such as detected signal structure, noise statistics and Channel State Information (CSI). Some of these information represent serious challenges which affect the spectrum sensing as we will see in Sec. 1.3.

\subsection{Spectrum Sensing Challenges}

Some technical challenges of cognitive radio spectrum sensing make it a difficult and even unreliable task unless precautions and measures are taken.

The main reason for these challenges is that there is no signaling (communication) 


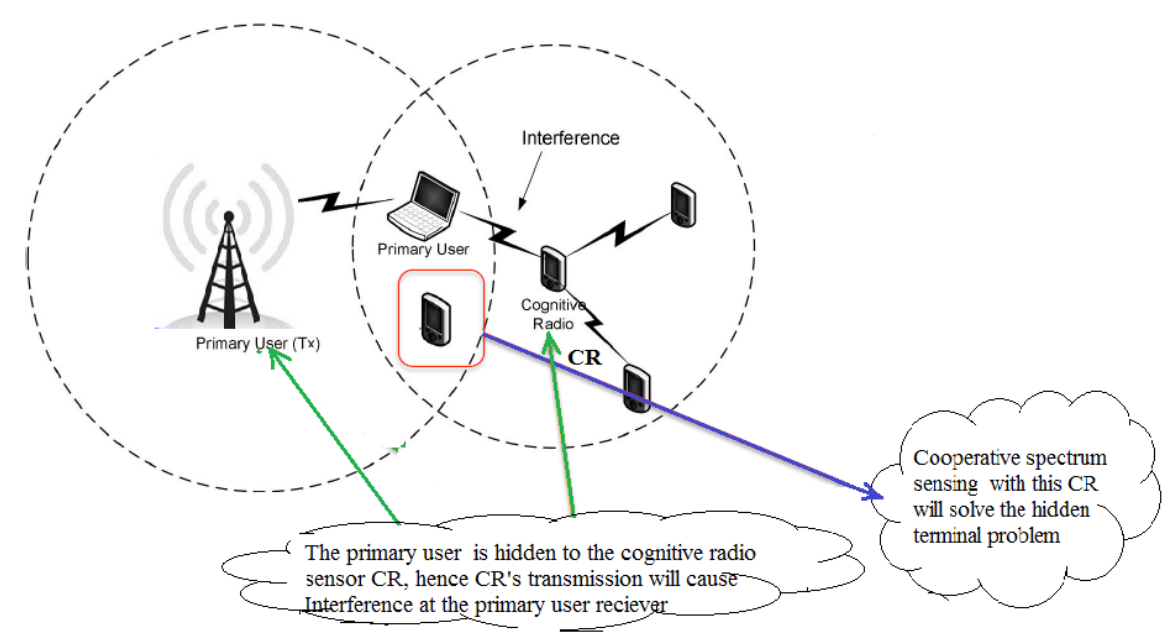

Figure 1.4: Hidden terminal problem in Cognitive Radio Spectrum Sensing.

between the cognitive sensor and the licensed primary user. As a result, the cognitive node must rely on detecting a weak signal coming from the primary user, which is the main task of the cognitive radio concept. Therefore, the cognitive sensor must be equipped with a receiver with high sensitivity margin compared with the primary user receiver.

The inherit hidden terminal problem in wireless communication [11] is another challenge in the spectrum sensing process (Figure. 1.4). It is defined as the failure of the secondary user to sense the primary user signal due to either receiver uncertainty or severe fading environment. Consequently, the secondary user will assume the absence of the signal of the licensed primary user and start access the spectrum by transmitting. Another serious challenge for reliable spectrum sensing is the multipath fading, the main concern and focus of this thesis.

Typically in a multipath fading or shadowing channel, the propagating signal experiences sharp fluctuations and might become weak. However this does not necessary mean 
that the primary user is not active or located out of the cognitive interference zone [12]. Hence, the knowledge of the fading envelop statistics is necessary to combat its harmful impact against any interference and to maximize the spectrum usage, especially when the signal power becomes comparable with the noise power [13].

Correlation among multiple receiving antennas is another issue impairs spectrum sensing when independent fading branches are assumed. This issue arises when diversity combining technique is employed to combat the multipath fading impact [14-16]. Considering independent antenna branches is often an invalid assumption, especially, with the increasingly closely spaced antennas in small mobile units [17]. However, the effect of correlation among antenna branches depends on the type of deployed diversity combining technique.

Sensing time is another issue in cognitive spectrum sensing. While accessing the vacant spectrum, the cognitive sensor must keep sensing the spectrum in order to exit once the primary user starts transmitting to avoid any interference with it [12]. Typically, a cognitive radio unit cannot sense and transmit simultaneously, therefore it has to periodically sense and transmit in a time slotted manner. Consequently, this switching between sensing and transmitting modes results in throughput reduction and inefficient spectrum usage.

Due to these challenges, it becomes clear that sensing is highly restricted and limited. Accordingly, these challenges need to be addressed since reliable and high efficiency performance is of the top priority of spectrum sensing process. 


\subsection{Motivation}

Developing an accurate model for detection probability in realistic sensing environments is vital in cognitive radio network in order to prevent the secondary user from harmfully interfering with the primary user while not missing out potential spectrum opportunities. Multipath fading and correlation among receiving antenna branches are the major cause of the sensing impairments.

Diversity combining is usually implemented in advanced wireless communication so as to address the multipath fading issue. In this technique, two or more copies of the desired signal are appropriately combined to maximize the Signal-to-Noise-Ratio (SNR) or some other figure of merit. This approach effectively mitigates the impairments of multipath fading and yields the best possible benefit when the time varying multipath channel seen by each antenna branch is independent [18]. However, this is not often the practical case and the maximal theoretical diversity gain is not attainable. Assuming independent antenna branches is often invalid where correlation coefficients in range (0.6-0.8) were observed. This observation were confirmed for multipath diversity over frequency-selective fading scenarios, especially, with the increasingly closely spaced antennas in small mobile units [19]. Thus, it is important to examine and combat the correlation impact on the diversity performance.

Furthermore, it is well-known that diversity combining techniques are different in their optimality and complexity[19]. Therefore, a tradeoff between these two issues needs to be achieved in order to maximize the performance of the diversity technique while keeping the add-on complexity of the wireless communication system at the minimum. Motivated by these objectives, we study different diversity combining techniques and 
compare their performances in different fading and correlation sensing scenarios.

Decorrelation is one of the effective techniques employed to combat the correlation adverse impact in wireless communications. Karhunen-Loeve Transformation (KLT) is often employed in this technique, consequently, the covariance matrix knowledge is required to perform the diagonalization [20]. In some special cases when the dual diversity is employed, decorrelation can be performed easily by adding and subtracting the diversity branches. However, this requires the evaluation of the statistics of the sum and the difference of correlated bivariate due to the random nature of the wireless channel. This also motivates us to study the decorrelation technique and to propose an effective and simple diversity decorrelator.

\subsection{Related Work}

In this section, we review the up-to-date work of the spectrum sensing in correlated multipath fading environments. We also review the work related with decorrelation as an effective solution for combating the correlation among multipath diversity branches.

To ensure a better detector performance, hence, a high probability of detection and low false alarm, the sensing process has to take into account the real signal propagation environment, thus the effect of the multipath fading. Usually, this is done by averaging the detection probability over the PDF of the corresponding fading channel and employing the appropriate diversity combining technique. Furthermore, the correlation among diversity fading branches in multi-receiving antennas is another issue impairs sensing process[19]. Therefore, it has to be considered in the investigations of the signal detection towards more realistic and accurate in the spectrum sensing. 
The core of CRNSS is the classic signal detection theory which is extensively investigated in literature. Urkowitz [21] was the first to investigate the unknown deterministic signal detection in a flat band-limited Gaussian noisy channel employing an Energy Detector (ED). In this technique, the received energy samples are squared, integrated and then compared with the decision threshold. This detection problem has been revisited for the first time by Kostylev [22] for more realistic situations of averaging the detection probability over fading channels. In this work, the Rayleigh, Nakagami and Rician fading distributions were considered and closed-form expressions for probability of detection $\left(P_{D}\right)$ and probability of false-alarm $\left(P_{F}\right)$ were derived for the Rayleigh fading channel. However, a numerical integration for Nakagami- $m$ fading channel and an infinite summation for the Rician fading channel were needed for $P_{D}$ calculation. Furthermore, only Nakagami- $m$ integer parameter values were considered.

In [23] and [24], alternative analytic approaches to that in [22] were introduced and extended work considering some diversity combining techniques were employed. In [23], independent and identically distributed (i.i.d.) L number of Rayleigh fading branches with EGC, SC and SSC were considered and corresponding average detection probabilities were derived. However, dual branches were considered for SSC diversity technique. In [24], the authors extended their work for i.i.d. L number of Additive White Gaussian Noise (AWGN) and Rayleigh with Square-Law Combining (SLC) and Square-Law Selection Combining (SLS). Furthermore, $L$ non-independent and identically distributed (n.i.i.d.) with SLS was considered. In fact the derived detection probabilities are limited to an integer-valued shape parameter $(m)$ and to unity Time Bandwidth Product (TW) in the decision variable $u=T W$. In [25], closed-form expressions were presented for $P_{D}$ and $P_{F}$ for multiple antenna spectrum sensing employing MRC and SC techniques assuming L-i.i.d. Rayleigh branches fading. 
In [26], an Orthogonal Frequency-Division Multiplexing (OFDM) based Cognitive Radio Sensor (CRS) equipped with multiple antenna and employing SLC was investigated assuming i.i.d. fading branches and alternative approach to that in [27] was introduced. Investigation demonstrated improved detection compared with single antenna reception. Again, assuming i.i.d., a novel spectrum sensing technique was proposed in [28]. This technique consists of multiple antenna CRS which are equipped with multiple ED devices and work collaboratively to perform spectrum sensing. Numerical analysis for Suzuki fading channel showed improved detection probability compared with the conventional sensing technique. Spectrum sensing with multiple antenna exponentially correlated in Rayleigh fading channel was investigated in [29]. Detection parameters were derived employing Central Limit Theorem (CLT) in order to approximate the ED statistics. Investigation showed the degradation in sensing performance due to the correlation among the antennas. The drawback of this approach is that CLT may not hold due to unavailability of large number of signal samples.

In [30-34] closed-form expressions for average detection probability $\left(\bar{P}_{D}\right)$ were introduced for i.i.d. Nakagami- $m$ and Rician fading branches employing different diversity combining techniques. In these works, $\operatorname{SSC}(L=2)$, EGC $(L=2,3$ and $L \geq 4)$, SC $(L \geq 2)$, and for MRC, SLC and SLS, $L$ number of diversity branches were investigated. For MRC, authors employed both PDF and Moment Generating Function (MGF) approaches. Again, using MGF approach for $L$-Nakagami- $m$ correlated branches with SLC, closed-form expressions were derived for constant, exponentially and a linear array of 2, 3 and 4 arbitrarily correlated antennas [35]. Investigations including correlation degree, TW, number of antennas and the fading severity and their impact on detection were done. ED cooperative spectrum sensing over i.i.d. Nakagami-m frequency-selective fading channel was investigated in [36] and corresponding average 
detection probability was evaluated. Investigation results showed improved detection performance compared to a flat-fading scenario due to both the additional multipath $L$ and the parameter $m$.

In [37], i.i.d. L Nakagami- $m$ lognormal composite ${ }^{1}$ channel branches was investigated without and with SLC and SLS diversity, and MGF based approach was derived. The author claimed that the proposed approach could be employed for correlated Nakagami-lognormal composite channel, however, he didn't show how this would be possible. In [38], detection performance was investigated over generalized fading environment employing the MGF approach and independent but not identically distributed (i.n.i.d.) branches were assumed with SLC, SLS and MRC diversity schemes. However, only MRC with constant correlated branches scenario was considered and closed-form approximations were derived in these investigations. In [39], spectrum sensing employing $p$-norm Detector ${ }^{2}$ was investigated for a single and multiple CRS in path-loss, shadowing, multipath fading and random network interference environments assuming i.i.d. fading branches.

In [40], an expectation-maximization based cooperative spectrum sensing approach $[41,42]$ was proposed considering cognitive secondary users equipped with multiple antenna. Decision statistics and then corresponding detection probabilities were derived assuming independent antennas reception. In [43], a cooperative spectrum sensing employing ED and named as multi-selective scheme was proposed. Assuming i.i.d. Rayleigh fading branches, detection probabilities were derived and improved performance compared with other diversity techniques such as SLS and SC was shown.

\footnotetext{
${ }^{1}$ Nakagami-lognormal composite channel model results from the combination of Nakagami- $m$ fading and lognormal shadowing.

${ }^{2} p$-norm Detector is a generalized version of the classical ED where the squaring operation is replaced by a power $p>0$, thus $y=\frac{1}{N} \sum_{i=1}^{N}\left|x_{n}\right|^{p}$.
} 
In [44], i.i.d. L Mixture Gamma Distribution (MG) fading channel branches ${ }^{3}$ was investigated with MRC, SLC and SLS diversity and corresponding average detection probability expressions were derived.

To the best of our knowledge, reviewing the literature reveals that most previous works which investigated the detection probability in fading channels assumed i.i.d. diversity branches. In many cases, the assumption of uncorrelated branches is not valid, specifically, in massive antenna systems, where insufficient antenna spacing in small-size mobile units equipped with space and polarization antenna diversity are employed. For multipath diversity over frequency-selective channels, correlation coefficients up to 0.6 between adjacent paths were observed [19].

Consequently, towards more realistic sensing scenario, more accurate detection and better primary user protection against interference, we consider correlated Nakagami- $m$ fading branches in our investigations of the spectrum sensing in this thesis. We implement a unified approach of performance analysis employing MRC, EGC, SC and SSC diversity combining techniques. We derived closed form expressions for detection probability considering this more realistic sensing scenario with some more general and simpler alternative expressions. Through numerical analysis, we show the invalidity of assuming independent fading branches, especially in deep fading and high correlation sensing scenarios. Furthermore, a comparison of performance analysis between closely combining techniques shows that in deep fading and high correlation regimes, simpler technique could replace the more complex one. Thus, reducing the cost and complexity while maintaining the same performance.

\footnotetext{
${ }^{3} \mathrm{MG}$ distribution $[45,46]$ has been proposed as an alternative model to various generalized and composite fading channels, such as, lognormal, Weibull, Rayleigh-lognormal, Nakagami-lognormal, K, $K_{G}, \eta-\mu, \kappa-\mu$, Hoyt, and Rician channels.
} 
In order to combat the correlation among diversity branches, most recent studies that address correlation describe various methods for improving efficiency of diversity branches. Decorrelation receivers have been studied in the literature. KLT is often employed for this purpose. In [47], decorrelation of MRC diversity branches was studied but, results showed no improvement compared with correlated one. Considering Rayleigh fading channels, authors in [48] employed KLT of $N \times N$ dimensional channel correlation matrix (and dual correlation in [49]) to create uncorrelated virtual antennas for SC and SSC techniques. Simulation-based analysis showed an enhanced performance in terms of decreasing outage probability and switching rate, while increasing the average SNR. In [50] also, KLT-based decorrelation was employed for SC and EGC in Rayleigh fading channels and MRC error analysis was applied easily to the decorrelator outputs. However, in general, knowledge of the covariance matrix is required to diagonalize it using KLT approach [20] which in turn requires CSI.

However, when only dual diversity $(L=2)$ is employed, decorrelation can be performed easily by adding or subtracting the signals received by correlated branches [51]. This yield to a relatively simple, feasible solution. Nevertheless, an evaluation of the statistics of the sum and the difference of a correlated bivariate is required to implement this stochastic dual decorrelator receiver due to the random nature of the wireless channel. In [51], decorrelation was done through adding and subtracting the diversity branches. It was performed for dual SC, and SSC diversity branches in Rician and Rayleigh fading channels and improvement was observed in Bit Error Rate (BER), average SNR and outage probability. However, no improvement observed for EGC and SLC techniques. In [52], a similar approach to that in [51] was used for a dual branch MRC receiver operating in correlated Hoyt fading channels. Then results were employed to simplify computation of the Symbol Error Rate (SER) in the 
investigated problem. In [53], decorrelation was employed to simplify the SLC-based energy detection analysis in correlated Rayleigh and Rician fading channels. In papers [54-57], dealing with decorrelation, numerical analysis showed improved Signal-to-Interference-Noise-Ratio (SINR) and system capacity.

Our approach in combating the correlation among fading branches employs the same decorrelation concept as in [51], however, it considers Nakagami- $m$ distribution, the more universal fading distribution. More importantly and in contrast to the work in [51], our approach derives the statistics necessary for decorrelation form fundamental principles by deriving the PDF and the CDF of the difference of correlated Nakagami bivariate. Numerical analysis shows that the derived PDF could be perfectly represented by a semi-standard normal distribution, thus eliminating future derivations. Furthermore, our derived statistics can be used to solve the problem of self-interference in multicarrier systems as well.

The next section demonstrates our contributions in this thesis.

\subsection{Contributions}

The contributions of this thesis fall into two folds, analysis and solution (Figure 1.5). From an analysis point of view, this thesis investigates the impact of multipath fading and correlation among diversity branches problems on the performance of cognitive spectrum sensing. It implements a unified approach of performance analysis for spectrum sensing in correlated multipath fading branches. For a solution perspective, a decorrelator implementation at the receiver is proposed to combat the correlation among diversity branches. In particular: 


\section{Analysis Fold}

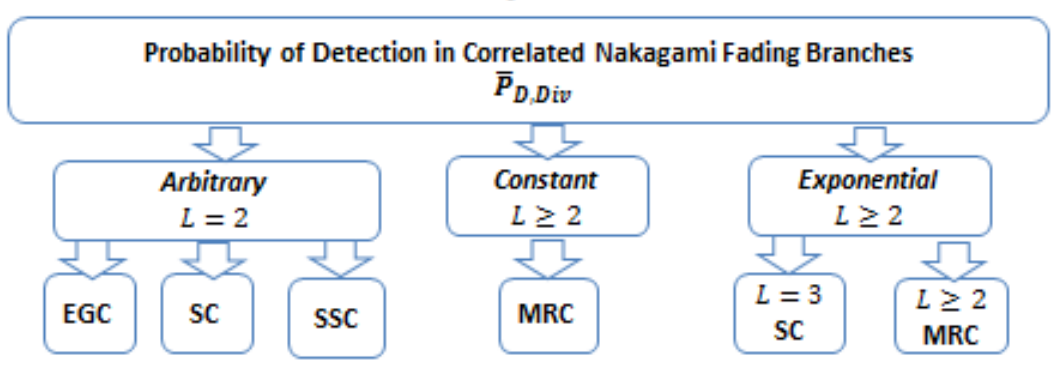

CLF Expressions for $P_{D}$ with some alternative, more general and simpler expressions for each sensing scenario

CLF Expression for Optimal Threshold $\gamma_{T}^{*}$ with SSC diversity case

\section{Solution Fold}

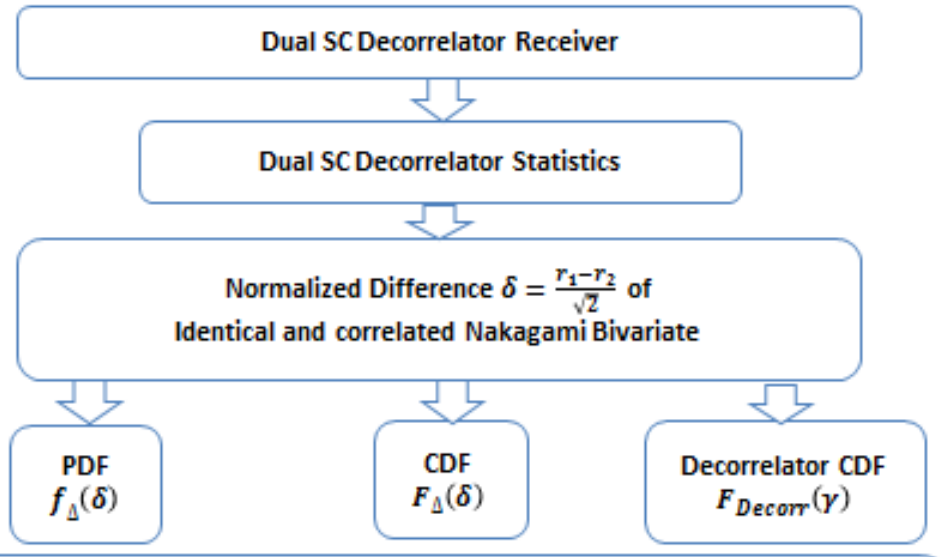

CLF Expression for PDF $f_{1}(\delta)$ with alternative, more \& simpler

$>$ CLF Expression for $\operatorname{CDF} \vec{F}_{\Delta}(\delta)$

$>$ CLF Expression for Decorrelator CDF $F_{D e c o r r}(\gamma)$

Derived Statistics can be used in self-interference for multicarrier systems

Figure 1.5: Summary of thesis contributions. 


\section{Analysis Fold}

- We investigate a more realistic sensing scenario through considering correlated Nakagami- $m$ fading branches

- We implement a unified framework of performance analysis for cognitive spectrum sensing with different diversity combining techniques including:

- Constant and exponentially correlated $L$-Nakagami- $m$ fading branches with MRC technique

- Exponentially correlated triple Nakagami- $m$ fading branches with SC technique

- Arbitrarily correlated dual Nakagami- $m$ fading branches with EGC, SC and SSC techniques

- We derive corresponding closed-form expression for average detection probability with some alternative, more general and simpler expressions for each sensing scenario

- We derive a corresponding closed-form expression for optimal threshold for SSC diversity case

- We perform a triple-level of analysis: individual, dual and comprehensive comparison performance analysis in order to verify the derived expressions and analyse the results 


\section{Solution Fold}

- We propose a decorrelated SC receiver for identical and correlated dual Nakagami- $m$ fading branches

* We derive expressions for the statistics of normalized difference of identical and correlated Nakagami- $m$ bivariate in order to facilitate the design of proposed receiver

* Our derived statistics can be used in the problem of self-interference for multicarrier systems

- To the best of our knowledge, we believe that the derived expressions are novel in the literature

\subsection{Thesis Outline}

The remainder of this thesis is organised as follows. In the next chapter, we review some sensing techniques, namely, signal detection techniques with focusing on the widely used ED technique. Furthermore, we review the two wireless channels models, the ideal and multipath fading models. We next describe the correlation among multi-antenna receivers resulting from employing diversity technique in order to combat multipath fading impact on detector performance. We conclude the chapter by describing the ED system model employed in this thesis under our more realistic sensing scenario through considering non-independent fading diversity branches. In chapter 3 , we investigate constant and exponentially $L$ correlated Nakagami- $m$ fading branches with MRC diversity. We derive an exact closed-form expression for the 
average detection probability of each sensing scenario. We describe the performance analysis using Complementary Receiver Operating Characteristics (CROC) graphs to evaluate the derived expressions by studying the fading and correlation impact on the detection probability. In chapter 4 , we consider a detection scenario when EGC diversity technique is employed with dual correlated Nakagami- $m$ fading branches. We derive an exact closed-form expression for the average detection probability of each sensing scenario. We do performance analysis using CROC graphs to evaluate the derived expressions by studying the fading and correlation impacts on the detection probability. In chapter 5 , we consider a detection scenario when SC diversity technique is employed for dual and triple diversity branches. For triple case, an exponentially correlated branches are considered. We derive the average detection probability for dual and triple Nakagami- $m$ correlated fading branches with SC diversity. We do performance analysis using CROC graphs to evaluate the derived expressions by studying the correlation impact on the detection probability. In chapter 6 , we consider a detection scenario when SSC diversity technique is employed for dual diversity branches. We derive the average detection probability for dual Nakagami- $m$ correlated fading branches with SSC diversity. We do performance analysis using CROC graphs to evaluate the derived expressions by studying the correlation impact on the detection probability. In chapter 7, we propose a decorrelated-based SC receiver. We do so through investigating the problem of decorrelating SC receiver in identical and correlated dual Nakagami- $m$ fading branches. We derive the expressions for statistics of the normalized difference of Nakagami- $m$ bivariate which represent the foundation of our presented decorrelator. In chapter 8, we perform two levels of performance comparison, namely dual and comprehensive comparison in order to study the impact of multipath fading and correlation among antenna branches on the 
detection probability. Finally, we conclude this thesis in Chapter 9 and present some directions for future work. 


\section{Chapter 2}

\section{Spectrum Sensing in Correlated Multipath Fading Channels}

In this chapter, we review some sensing techniques, namely, signal detection techniques with focusing on the widely used ED technique. Furthermore, we review the two wireless channels models, the ideal and multipath fading models. We next describe the correlation among multi-antenna receivers resulting from employing diversity technique in order to combat multipath fading impact on detector performance. We conclude the chapter by describing the ED system model employed in this thesis under our more realistic sensing scenario through considering non-independent fading diversity branches. 


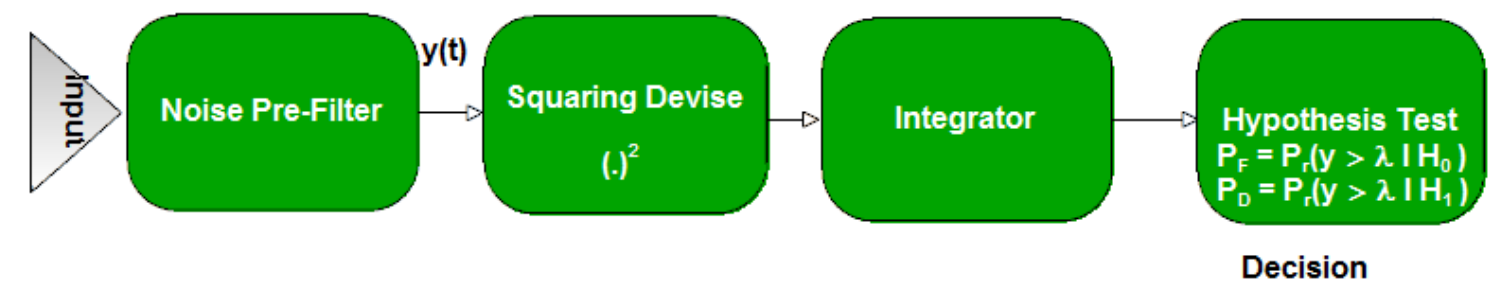

Figure 2.1: Energy detection spectrum sensing technique.

\subsection{Signal Detection Techniques}

Many signal detection techniques have been proposed in literature. The complexity level, implementation, advantages and drawbacks are described next.

\subsubsection{Energy Detection}

A non-coherent ED (with no synchronization) is a widely used and easiest sensing technique due to its robustness to unknown dispersive channel and fading. In this technique, no priori information about the detected signal is needed and it is optimal for detecting i.i.d. signals $[10,21,22,24,58,59]$. These features (especially, no priori information about the detected signal is needed ) make ED an attractive and perfect candidate for CRNSS since there is no signaling between the primary user and secondary user. However, the knowledge of noise statistics is still required for setting the threshold for hypothesis testing or these statistics must be estimated. 
Typically, in ED (Figure. 2.1), the received samples are filtered and passed to a squaring unit followed by integrator. The output is compared with a predefined threshold which is usually the noise power to make a decision of the presence or absence of the primary user signal.

\section{ED Drawbacks}

Since ED requires the knowledge of the noise statistics, its performance is vulnerable to noise uncertainty. To circumvent this problem, a pilot tone from the primary transmitter may be used to improve the ED accuracy. Furthermore, ED can only detect the presence of the signal, it cannot differentiate its type. Therefore, it is prone to the false detection triggered by unintended signals [60]. Another drawback of ED is that at low SNR spectrum sensing scenarios, ED is inefficient to discriminate the interference from the primary signal. In fact, ED's noise estimator is not good for small samples number $(N)$ in low SNR regimes. Hence, large number of samples is needed, consequently longer detection time.

A Generalized Likelihood Ratio (GLR) detector is proposed in [61] to improve ED performance and overcome some or all unknown parameters needed for optimal energy detection such as noise uncertainty, channel gain and primary user variance.

In our investigation in this thesis, we employ ED as a sensing technique in spite of its some aforementioned drawbacks since it is perfect candidate for CRNSS as we mentioned earlier. 


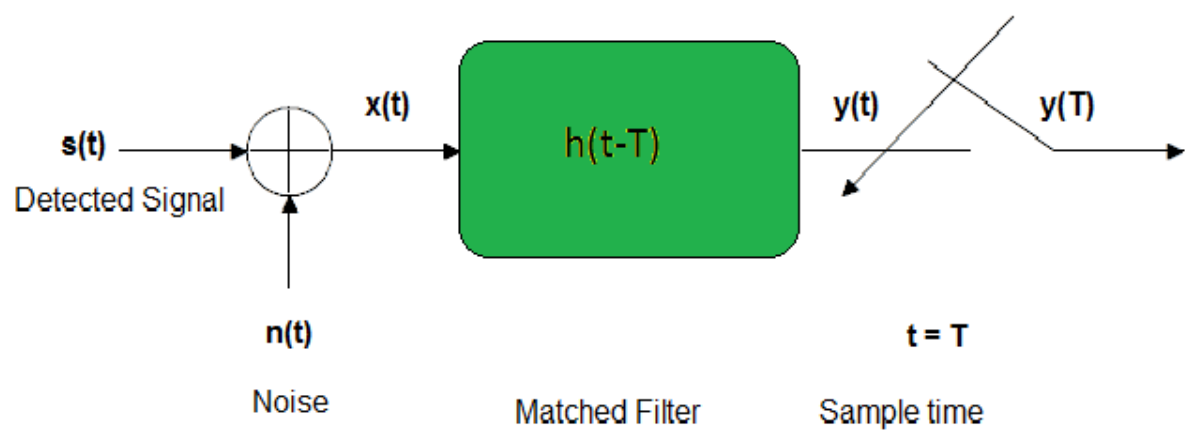

Figure 2.2: Matched filter detector block diagram.

\subsubsection{Matched Filter}

Matched filter is a coherent detector. It is optimal for detecting the priori known waveform information (such as: modulation type and order, pulse shape, and packet format) since it maximizes SNR and it has less required time to achieve high processing. A standard matched filter block diagram is shown in Figure. 2.2

\section{Matched Filter Detection Drawbacks}

Since matched filter depends on a prior knowledge of the detected signal, inaccurate signal waveform information results in poor detector performance, making it prone to detection errors. Consequently, this results in either low primary user protection against interference or low throughput which are both of high concern in a CRNSS process. 


\subsubsection{Cyclostationary Feature}

The main difference between the noise and the sensed primary user signal is that the latter has some non-random features such as double-sided (sinewave carrier), data rate (symbol period) and modulation type. Among these, the modulation which results in Cyclostationary feature due to built-in periodicity. Hence, the detected modulated signal has periodic autocorrelation and mean which can be detected by analyzing the spectral correlation function.

In fact, the noise is a wide-sense stationary signal with no correlation, while the modulated signal is Cyclostationary with spectral correlation due to the embedded redundancy of signal periodicity. Consequently, primary signal can be differentiated from the noise. Hence, the non-coherent (no synchronization required) Cyclostationary feature detector outperforms ED in differentiating noise from the primary user signal for its robustness against the noise power uncertainty $[60,62,63]$.

\section{Cyclostationary Feature Detection Drawbacks}

This technique requires a priori knowledge about the detected signal. Furthermore, it is computationally complex and requires significantly long observation time. Hence, it makes it impractical for many situations of cognitive spectrum sensing.

\subsection{Wireless Channel}

Typically, the channel between the transmitter and receiver has a great impact on the propagated signal. Depending on its type whether ideal or non-ideal, it may change 
the signal characteristics such power, phase and others. In general, there are two scenarios of wireless system modeling.

\subsubsection{Free-Space Model}

In this model, the channel is considered ideal if its characteristics are not specified [64]. Accordingly the ideal channel means the following:

1. It is free of any object that might absorb or reflect the propagated signal energy, hence, only one Line-of-Sight (LOS) path.

2. The channel atmosphere behaves perfectly uniformly and the medium is nonabsorbent.

3. Earth reflection coefficient is negligible as it is considered far away from the propagated signal.

Actually, in most cases this idealized channel is not realistic especially when signal propagates in the atmosphere, close to the ground and in congested medium where many obstacles are there such as hills, vegetation or building. Consequently, the transmitted signal will arrive the receiver via multiple paths which is called multipath propagation. Accordingly, it is necessary to consider a more comprehensive model that takes into account these considerations since free-space model is insufficient to describe the real channel behavior as we will see in the next section.

\subsubsection{Multipath Fading Model}

Usually, the transmitted signal degrades during its prorogation from the transmitter to the receiver due to mainly the time-variant impulse channel response. Moreover, the 


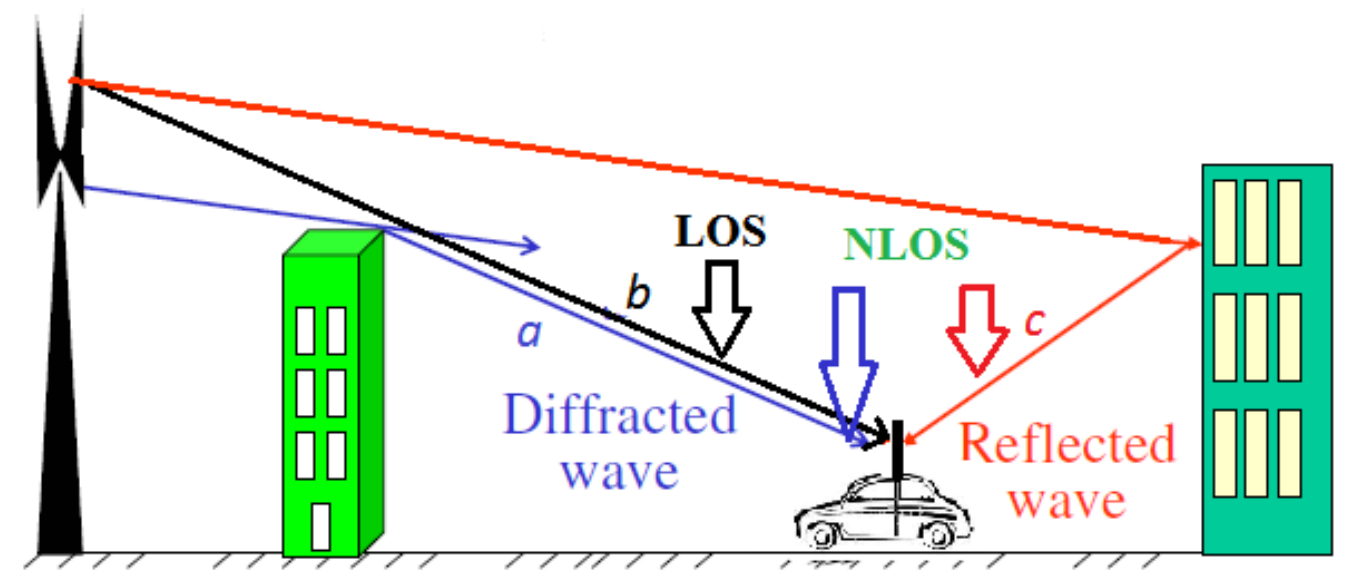

Figure 2.3: Fading in wireless communication channel.

objects close to the signal such us the ground and in the space between the transmitter and the receiver cause the signal to reflect, scatter, diffract and be absorbed during its propagation in the channel (Figure 2.3). Consequently, the multiple copies of the transmitted signal arrive at the receiver in addition to the direct path LOS.

When there is No-LOS, the received signal will be just multipath replica of the transmitted signal. In both cases, fading will occur to the received signal causing fluctuating in its amplitude, phase and arrival angle, however, in No-LOS is more severe. This degradation in the transmitted signal manifests as distortion and loss SNR, giving rise to socalled multipath fading [64]. Typically, limited number of paths (4 - 24) are used to model the radio channel. 


\subsection{Spectrum Sensing with Diversity Combining}

To combat the loss of SNR, different diversity techniques include time diversity, frequency diversity, spatial diversity and polarization diversity. Among them is the spatial diversity technique which will be our focus since we basically consider a multiple antenna spectrum sensing.

Typically, multiple antenna are spaced (ideally separated by one half wavelength or more) and connected to a common receiving system so-called, SIMO as shown in Figure 2.4. In this receiving system, when one antenna sees a signal very week or even null, another antenna may see a signal peak. Consequently, the receiver is able to select the antenna with the best signal or combine all branches or perform any appropriate decision depending upon the employed diversity technique as we will see later.

The equivalent low pass of the received signal of $l^{\text {th }}$ branch could be expressed as

$$
r_{l}(t)=\alpha_{l} e^{-j \theta_{l}} \delta\left(t-\tau_{l}\right) s(t)+n_{l}(t), \quad l=0,1,2 \cdots L_{P}
$$

where $n_{l}(t), L_{P}, l$ and $\delta($.$) denote noise, number of resolvable paths { }^{1}$, channel index and Dirac delta function, respectively. Also the random fading parameters $\left\{\alpha_{l}\right\}_{l=1}^{L_{P}}$, $\left\{\theta_{l}\right\}_{l=1}^{L_{P}}$ and $\left\{\tau_{l}\right\}_{l=1}^{L_{P}}$ are respectively; amplitude, phase and time delay where the first path of the signal replica has $(\tau=0)$ and is related to the ratio of maximum delay spread $\tau_{\text {max }}$ to the symbol time $T_{s}$. Due to LOS, the first arriving path in the impulse response may experience lower amount of fading than the subsequent paths. While

\footnotetext{
${ }^{1}$ Here $L_{P}=N$, number of diversity receiving antennas.
} 


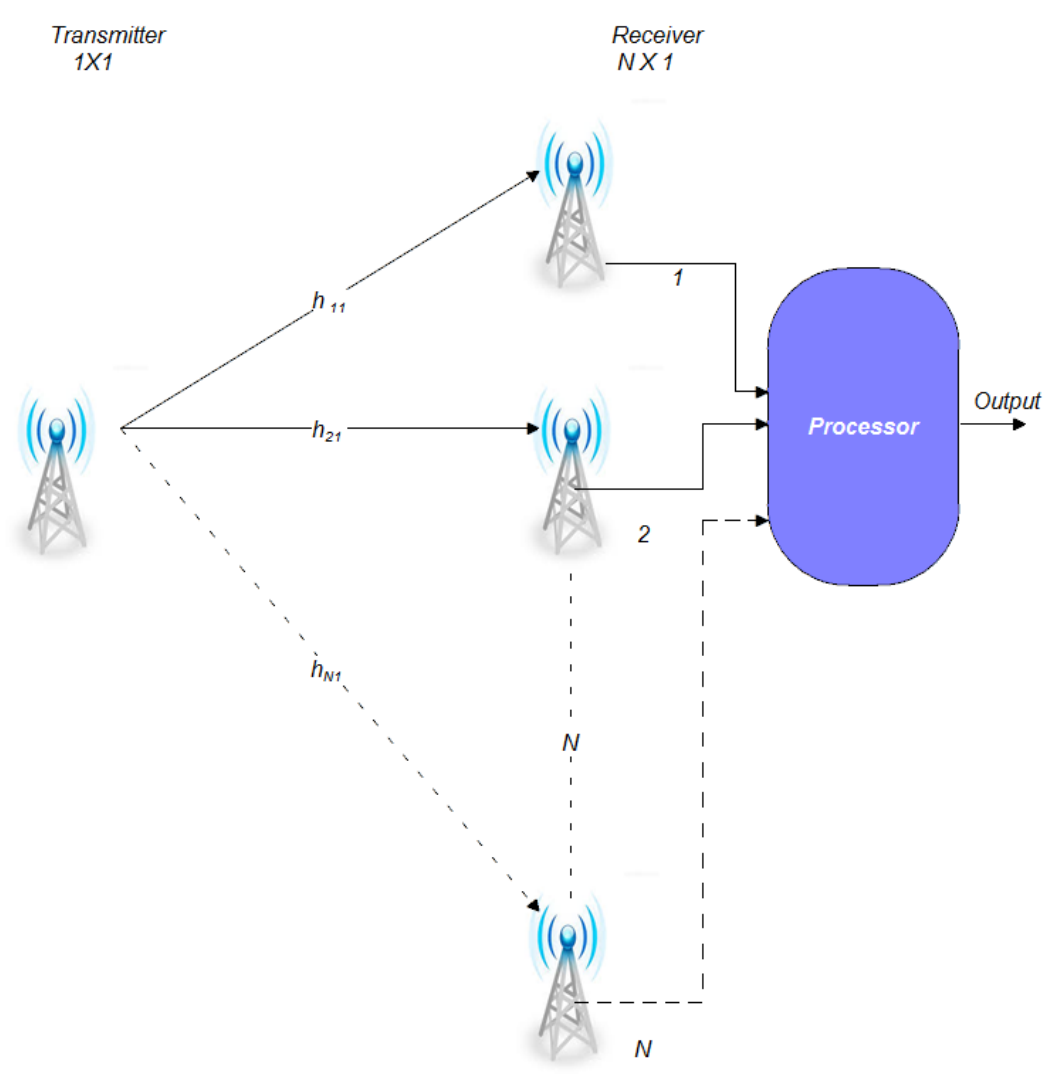

SIMO: $N X 1$

Figure 2.4: SIMO system.

the last arriving paths experience higher fading amounts due the decrease in specular power component with respect to the delay. Hence, out of $L_{P}$-branches, $L_{P}$-replicas of the transmitted signal are obtained

$$
r=\left[r_{1}(t), r_{2}(t), \cdots, r_{L_{P}}(t)\right]
$$

In diversity combining application, a multilink channel model is introduced where an 
$L$ replicas of the transmitted signal propagated in the fading channel are combined in order to increase the overall SNR. Hence, compensating the loss in SNR occurred due to time-variant impulse channel response and due to other causes as we mentioned earlier.

For a multicopy of received signal, the instantaneous SNR is $\gamma_{l}=\frac{\alpha_{l}^{2} E_{s}}{N_{l}}$ where $\alpha_{l}, E_{s}$, and $N_{l}$ are; fading amplitudes of the $l$-th tap, energy per symbol and the one-sided noise power spectral density respectively. The average SNR per symbol is $\bar{\gamma}_{l}=\frac{\Omega_{l}^{2} E_{s}}{N_{l}}$ where $\Omega_{l}$ denotes the mean-square of the random variable channel amplitude $\Omega_{l}=\overline{\alpha^{2}}$ [19]. Regarding the type of the employed channel, consequently, the distribution of the corresponding channel gain coefficient, we will consider in this thesis the Nakagami- $m$ distribution fading channel. Furthermore, MRC, EGC, SC and SSC will be employed in our investigations as combining diversity techniques.

Diversity combining techniques differ in their optimality and complexity. Some combining techniques process all diversity branches such as MRC and EGC techniques. Others, such as SC and SSC techniques process only one diversity branch. For MRC, it has the optimal performance in combating the multipath fading in wireless communication systems. However, it is the most complicated diversity technique since the full knowledge about the received signal is required for maximizing the total SNR. The suboptimal EGC is less complicated since less knowledge about the received signal is required as we will see later in discussing the detection probability with EGC diversity technique. SC combiner selects only the highest SNR branch, therefore, it has less complexity and performance than the two previous techniques since it processes only one branch. Likewise, SSC processes one branch only, however it holds on this branch until its SNR drops below a predetermined threshold then it 
switches to another branch. Therefore, it is the least complicated technique since no continuous monitoring for the SNR's branch is required as in SC. However, it has the lowest combiner performance.

\subsection{Correlation in Wireless Channels}

Channels due to scattering in the propagation paths in multipath wireless communication environment are often not independent from each other. Consequently, these channels are related to each other with different degrees which gives rise to socalled, correlation or more specifically, spatial correlation. Furthermore, especially in cognitive spectrum sensing, the large distance between the primary user transmitter and the CRS receiver (typically $d>100 \mathrm{~km}$ in IEEE 802.22 WRAN systems) generates a small received-channel angular spread value at the CRS receiver [65] resulting in highly correlated channels. Due to the random nature of the wireless communication channel, random variables are good representation for its parameters.

The correlation coefficient between two random variables $X$ and $Y$ is defined as

$$
\rho_{X, Y}=\frac{C O V(X, Y)}{\sqrt{\sigma_{X}^{2} \sigma_{Y}^{2}}}=\frac{E[X Y]-E[X] E[Y]}{\sqrt{\sigma_{X}^{2} \sigma_{Y}^{2}}}, \quad-1 \leq \rho_{X, Y}<1
$$

where $C O V(.,$.$) denotes the covariance, E[$.$] the expectation and \sigma^{2}$ the variance.

The covariance is zero for independent random variables, hence, they are orthogonal. According to the antennas geometric distribution, spatial correlation between each pair of antennas could be modeled as a constant, exponential or linear array of arbitrary correlation $[66,67]$. 
For constant correlation matrix model, antennas are closed enough and geometrically distributed in a circular array resulting in equal correlation between each pair of antennas. Consequently, for $N$-correlated antennas, the entries of the corresponding correlation matrix $\Sigma_{\text {Const }}=\left[c_{i, j}\right]$ will be $c_{i, j}=1$ for $i=j$, and $c_{i, j}=\rho$ for $i \neq$

$j[66]$.While exponential correlation model represents equispaced antennas, consequently, correlation between antenna pair decreases as the separation between them increases [68]. Therefore, the corresponding correlation matrix entries will be $\boldsymbol{\Sigma}_{\text {Expon }}=\left[\rho^{|i-j|}\right]$. In fact, exponential correlation is more general and realistic than constant correlation model and has wide successful applications in wireless communications $[16,66,69,70]$. The third model is the linear array of arbitrary correlation where antenna elements are situated in a linear configuration. In this model, the correlation is said to be arbitrary depending on some factors such as incident angle, antennas spacing or height, hence, the correlation matrix is Toeplitz [35].

\subsection{Signal Detection in Correlated Multipath} Fading

Cognitive radio spectrum sensing aims to detect reliably the hole (unused band) in the spectrum to opportunistically reuse it in the absence of the licensed primary user. Primary user is defined as the licensed user that owns that detected band, while the secondary user who senses the spectrum to find that vacant band in order to reuse it in the absence of the licensed primary user.

In fact, the core of the cognitive radio spectrum sensing is the classical problem of detecting a signal in a noisy channel [21] which has been researched extensively. The 
system model of this signal detection problem will be discussed in details in the next section.

\subsubsection{System Model}

We consider the classic problem of detection theory [21] which is that of unknown deterministic signal over flat bandlimited AWGN channel in cognitive radio spectrum sensing environment. Typically, an ED is employed since it is the simplest detection technique and more convenient to fit CRNSS systems where the secondary user (i.e. CRS) has no knowledge about the sensed primary user signal. For the sake of brevity, we refer the reader to Sec. 2.1.1 where the ED's principle of operation has been described. However, more details regarding the decision statistics will be given in this section.

Let $x(t)$ be the received data vector as

$$
x(t)=h s(t)+n(t)
$$

where $h, s(t)$ and $n(t)$ denote the complex channel gain amplitude coefficient and is assumed constant during sensing time, detected signal and AWGN respectively. The noise is a low-pass AWGN process with zero mean and variance $N_{0} W$ where, $N_{0}$ and $W$ denote Power Spectral Density (PSD) of the Gaussian noise and signal bandwidth, respectively. The noise is assumed to be i.i.d. and uncorrelated with transmitted signal.

Two hypothesis are defined, namely $H_{0}$ and $H_{1}$ for the absence and the presence of 
the detected signal respectively, as follows

$$
x(t)= \begin{cases}n(t) & \text { if } H_{0} \\ h s(t)+n(t) & \text { if } H_{1} .\end{cases}
$$

The decision statistics of the energy detector is the output of the integrator over $T$ time interval and as follows [24]

$$
y \triangleq \frac{2}{N_{0}} \int_{0}^{T}|x(t)|^{2} \mathrm{~d} t .
$$

Therefore, the PDF of the decision statistics $y$ in $(2.6)$ is $[23,24]$

$$
p_{Y}(y)= \begin{cases}\frac{1}{2^{u} \Gamma(u)} y^{u-1} e^{\frac{y}{2}}, & \text { if } H_{0} \\ \frac{1}{2}\left(\frac{y}{2 \gamma}\right)^{\frac{u-1}{2}} e^{-\frac{2 \gamma+y}{2}} I_{u-1}(\sqrt{2 \gamma y}) & \text { if } H_{1},\end{cases}
$$

where $I_{\nu}($.$) denotes the \nu$ th-order of modified Bessel function of the first kind.

In (2.7), it is clear that the decision statistics has a central Chi-square distribution with $2 u$ degrees of freedom $\chi_{2} u^{2}$ in the absence of the primary user signal, i.e. the received samples are noise only. While it has a non-central Chi-square distribution with $2 u$ degrees of freedom $\chi_{2 u}{ }^{2}$ and $\psi$ non-centrality parameter $\chi_{2 u}(\psi)$ in the presence of the primary user signal.

Defining $\lambda$ as a decision threshold, the performance of ED is measured in terms of the 
detection probability $P_{D}$ and false alarm probability $P_{F}$, as follows

$$
\begin{aligned}
& P_{F}=\operatorname{Pr}\left(y>\lambda \mid H_{0}\right), \\
& P_{D}=\operatorname{Pr}\left(y>\lambda \mid H_{1}\right),
\end{aligned}
$$

Consequently, the false alarm and detection probabilities in AWGN channel are given respectively by [23],

$$
\begin{aligned}
P_{F} & =\frac{\Gamma\left(u, \frac{\lambda}{2}\right)}{\Gamma(u)}, \\
P_{D} & =Q_{u}(\sqrt{2 \gamma}, \sqrt{\lambda}),
\end{aligned}
$$

where $\Gamma(.,$.$) and Q_{u}(.,$.$) denote the upper incomplete Gamma function and Generalized$ Marcum Q-function, respectively.

Note that the detection probability expression in (2.11) is restricted to only integer values of $u$ since the probability of the decision statistics in (2.7) is derived only for even numbers, i.e. $2 u$ as stated in [23]. However, when the alternative Marcum-Q function is employed, $u$ could be half-odd integer $(u \in\{0.5,1,1.5,2,2.5,3, \ldots\}$, i.e. not restricted to integer values) [71]. Furthermore, the fading parameter $m$ in Nakagami channels might also be not restricted to integer values depending on the mathematical method employed to solve the integral in (2.12). This highlights the advantage of the alternative expressions which we derive later using alternative Marcum-Q function. 


\subsection{Problem Formulation}

The detection probability parameters (Sec. 2.5.1) are conditioned on a channel realization, i.e. they represent instantaneous detection probability since the sensing channel is considered ideal AWGN channel. Consequently, multipath fading is not considered. To remove this condition, we need to integrate this instantaneous detection probability ${ }^{2}$ over the PDF of the corresponding diversity fading channel distribution as follows:

$$
\bar{P}_{D, D i v}=\int_{0}^{\infty} Q_{u}(\sqrt{2 \gamma}, \sqrt{\lambda}) p_{\gamma D i v}(\gamma) \mathrm{d} \gamma
$$

The expression $\bar{P}_{D, D i v},(2.12)$ will serve as a general average detection probability expression for corresponding diversity fading channel.

Few work has been done to address this problem, namely signal detection in correlated multipath fading, however, in most cases, independent fading branches are assumed. Thus, ignoring correlation between fading paths making this assumption unrealistic too, as we indicated in Sec 1.5.

In fact, assuming independent branches is often invalid, especially, with the increasingly closely spaced antennas in small mobile units. Furthermore, the effect of correlation among antenna branches depends on the type of diversity combining technique employed to combat multipath fading impact. Actually these two above assumptions, namely, considering independent and AWGN channels do not reflect the real situation of the spectrum sensing process since multipath fading and correlation among receiving

\footnotetext{
${ }^{2}$ False alarm probability is not a function of SNR since it is for no signal transmission, therefore it will remain unchanged as in (2.10).
} 
antenna branches are the major cause for sensing impairments.

Motivated by these facts, hence, more primary user's protection against interference and maximum secondary user throughput, we will address these issues in CRNSS in the analysis fold of our work in this thesis.

In the following chapters, we will investigate the primary user signal detection problem considering a system model with constant and exponential correlation among $L$-Nakagami- $m$ fading branches employing PDF approach with MRC diversity.

Furthermore, we extend the investigations by considering identically and arbitrarily correlated branches for EGC, SC and SSC techniques in Nakagami-m multipath fading channels. Due to the complexity of the output SNR's PDF of suggested diversity techniques, we restrict our work with dual branches diversity. However, a triple identically and exponentially correlated fading branches with SC diversity is also considered and the corresponding closed-from expressions are derived. 


\section{Chapter 3}

\section{$L$ Correlated Nakagami- $m$ \\ Branches with MRC Diversity}

Few important issues are considered in our study; maximizing the dynamic spectrum sensing accuracy, maximizing secondary user throughput and reducing interference to the primary user. Typically, the throughput can be maximized by minimizing the false alarm probability since this indicates falsely that a vacant band (hole) of the spectrum is occupied. In other words, the real vacant band is mistakenly declared as an occupied band. As a result, secondary user throughput is decreased since not all the vacant bands are exploited.

On the other hand, protecting the licensed primary user's transmission from any interference with the secondary users transmission is achieved by reducing the missed detection probability $\left(P_{D m}\right)$. Since this mistakenly indicates no active primary user while actually there is. Consequently a vacant band is mistakenly declared. Minimizing this harmful interference is done by maximizing the detection probability which also 
will reduce missed detection probability since $P_{D m}=1-P_{D}$.

Typically, channel propagation between the transmitter and receiver has a great impact on the propagated signal. Multipath fading and shadowing are well known factors that they significantly impair sensing performances. These are typically addressed by diversity technique where, two or more copies of the desired signal are combined to maximize the average SNR. With spatial diversity, however, maximum diversity gain is attainable when the signals from multiple channels are independent (or orthogonal) [18]. The independency assumption among reception channels is often invalid due to the insufficient antenna spacing in ever shrinking mobile units. Therefore, it is important to examine the correlation impact on diversity performance. That is the focus of this work.

In this chapter, we investigate constant and exponentially $L$ correlated Nakagami- $m$ fading branches with MRC diversity. We derive an exact closed-form expression for the average detection probability of each sensing scenario. We describe the performance analysis using CROC graphs to evaluate the derived expressions by studying the fading and correlation impact on the detection probability.

\subsection{MRC Diversity}

In MRC technique as shown in Fig. 3.1, the received signals from all $L$ branches $\left\{y_{l}(t)\right\}_{l=1}^{L}$ are weighted (scaling factor) based on their individual SNRs, cophased and 


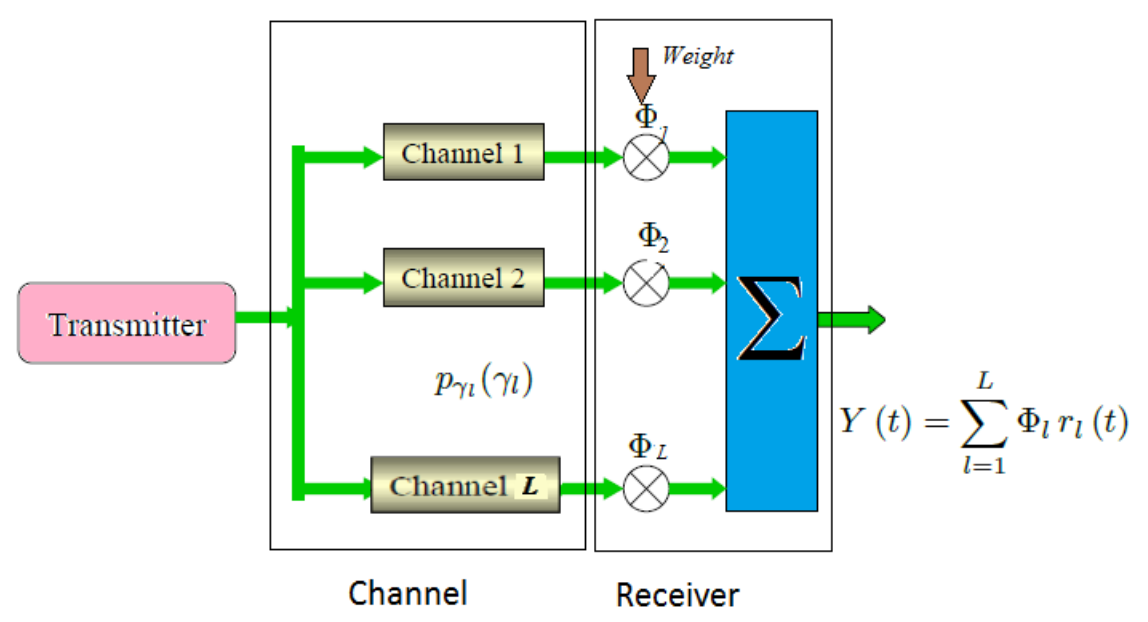

Figure 3.1: Maximal Ratio Combining Technique (MRC).

then summed, resulting in a new combined signal

$$
Y(t)=\sum_{l=1}^{L} y_{l}(t)=\sum_{l=1}^{L} \Phi_{l} r_{l}(t)
$$

where, $\Phi_{l}$ denotes the $l^{t} h$ assigned weight and $r_{l}$ is the received signal of $l^{\text {th }}$ branch given in (2.1). The effect of these scaling factors is that a strong signal carries a larger weight than a weak signal [4]. Therefore, MRC technique requires the knowledge of the channel amplitude and phase, making it the most complicated combining technique. This complexity comes from the assumption that the weighting factors and phase shifts are known exactly, which is not an easy task to implement in a digital receiver, since these weighting factors and phase shifts change in real-time. Moreover, receiver complexity depends on the number of resolvable paths. However it provides the maximum performance improvement in comparison with other diversity combining techniques through maximizing SNR at the combiner output[19,72], hence known CSI will be assumed. This CSI knowledge is justifiable since it may be available for 
the cognitive sensors over a control or broadcast channel [34]. Another assumption is that $L$ fading paths are n.i.i.d., i.e. correlated with equal average SNR $\bar{\gamma}$ with specific $\operatorname{PDF}\left(p_{\gamma_{l}}\left(\gamma_{l}\right)\right)$ depends on the statistics of complex gain channel.

In this thesis, we consider Nakagami- $m$ distribution in our investigations since this distribution is well suited to model both indoor and outdoor multipath fading channels. In fact, Nakagami- $m$ comprises, the one-sided Gaussian distribution $(m=0.5)$, the Rayleigh distribution $(m=1)$ and for $(m \rightarrow \infty)$, it converges to a non-fading AWGN channel [73].

The PDF of the individual complex gain channel corresponds to the PDF of Nakagami- $m$ univariate given [74] by

$$
f(\alpha)=\frac{2}{\Gamma(m)}\left(\frac{m}{\Omega}\right)^{m} \alpha^{2 m-1} e^{-\frac{m \alpha^{2}}{\Omega}}, \quad \alpha \geq 0
$$

where, $\Gamma($.$) denotes the Gamma function, \Omega=E\left[\alpha^{2}\right] / m=\frac{\overline{\alpha^{2}}}{m}$ is the mean value of the variable $\alpha$ and $m(m \geq 1 / 2)$ is the inverse normalized variance of $r^{2}$ which describes the fading severity. For $L$ MRC branches, the received signals are $\left\{y_{l}(t)\right\}_{l=1}^{L}$, therefore, the total effective SNR, $\gamma$ is obtained as [19]

$$
\gamma=\frac{E_{s}}{N_{0}} \sum_{l=1}^{L}\left|\alpha_{l}\right|^{2}
$$

where, $E_{s}$ denotes the signal power.

In multi-antenna reception, the correlation matrix depends on the geometric distribution of these antennas. For instance, a constant correlation model refers to closely placed diversity antennas. While an exponential correlation model refers to equispaced 
antennas. In the following sections, we will consider in our investigation both constant and exponential correlation models.

\subsection{Constant Correlation}

In this section, we will investigate constant $L$ correlated Nakagami- $m$ fading branches with MRC diversity. Assume equal correlation coefficient $\rho$; equal average $\mathrm{SNR} \bar{\gamma}$ and same fading parameter value $(m)$ among $L$ MRC Nakagami- $m$ fading branches. Then, the PDF of the total SNR $\gamma$ for closely placed diversity antennas under constant correlation is given [66]

$$
\begin{aligned}
& p_{\gamma_{M R C: C o n s t}}(\gamma)=\frac{1}{\left(\frac{\bar{\gamma}}{m}\right)(1-\rho)^{m(L-1)}(1-\rho+L \rho)^{m} \Gamma(L m)}\left(\frac{\gamma m}{\bar{\gamma}}\right)^{L m-1} \\
& \times \exp \left\{-\frac{\gamma m}{\bar{\gamma}(1-\rho)}\right\}_{1} F_{1}\left(m, L m ; \frac{L m \rho \gamma}{\bar{\gamma}(1-\rho)(1-\rho+L \rho)}\right), \quad 0 \leq \rho<1 .
\end{aligned}
$$

where ${ }_{1} F_{1}(., . ;$.$) denotes the Confluent Hypergeometric function and is defined in [$ [75], Eq. (15.1.1)] as

$$
{ }_{1} F_{1}\left(a_{1}, b_{1} ; x\right)=\frac{\Gamma\left(b_{1}\right)}{\Gamma\left(a_{1}\right)} \sum_{n=0}^{\infty} \frac{\Gamma\left(a_{1}+n\right) x^{n}}{\Gamma\left(b_{1}+n\right) n !} .
$$

For simplicity, we can write (3.4) as

$$
p_{\gamma_{M R C: \text { Const }}}(\gamma)=\frac{A}{D} \gamma^{L m-1} e^{-B \gamma}{ }_{1} F_{1}(m, L m ; C \gamma)
$$


where the constants $A, B, C$ and $D$ are respectively defined as follows:

$$
\begin{aligned}
A & =\left(\frac{m}{\bar{\gamma}}\right)^{L m}, \\
B & =\frac{m}{\bar{\gamma}(1-\rho)}, \\
C & =\frac{L m \rho}{\bar{\gamma}(1-\rho)(1-\rho+L \rho)}, \\
D & =(1-\rho)^{m(L-1)}(1-\rho+L \rho)^{m} \Gamma(L m) .
\end{aligned}
$$

Therefore, the average detection probability for $L$ MRC correlated Nakagami- $m$ branches with equal $m, \rho$, and $\bar{\gamma}$ could be obtained by substituting (3.6) in (2.12) yields

$$
{\overline{P_{D}}}_{R R C \text { Const }}=\frac{A}{D} \int_{0}^{\infty} Q_{u}(\sqrt{2 \gamma}, \sqrt{\lambda}) \gamma^{L m-1} e^{-B \gamma}{ }_{1} F_{1}(m, L m ; C \gamma) \mathrm{d} \gamma
$$

Using the alternative expression of Marcum Q-function for not restricted to $u$ integer values given in [[76], Eq. (4.63)], $Q_{u}(\sqrt{2 \gamma}, \sqrt{\lambda})$ in $(3.11)$ could be written as

$$
Q_{u}(\sqrt{2 \gamma}, \sqrt{\lambda})=1-e^{-\frac{2 \gamma+\lambda}{2}} \sum_{n=u}^{\infty}\left(\frac{\sqrt{\lambda}}{\sqrt{2 \gamma}}\right)^{n} I_{n}(\sqrt{2 \lambda \gamma})
$$

where $I_{\nu}($.$) denotes the \nu$ th-order of the modified Bessel function of the first kind. Substituting (3.12) in (3.11) and using the definition of the PDF as

$$
\int_{0}^{\infty} p_{\gamma}(\gamma) \mathrm{d} \gamma=1
$$


therefore, (3.11) becomes

$$
\begin{array}{r}
{\overline{P_{D M R C: C o n s t}}}_{M}=1-\frac{A}{D} e^{-\frac{\lambda}{2}} \sum_{n=u}^{\infty}\left(\frac{\sqrt{\lambda}}{\sqrt{2}}\right)^{n} \int_{0}^{\infty} \gamma^{L m-1-\frac{n}{2}} e^{-\gamma(1+B)} I_{n}(\sqrt{2 \lambda \gamma}) \\
\times{ }_{1} F_{1}(m, L m ; C \gamma) \mathrm{d} \gamma
\end{array}
$$

Using (3.5), the series representation of the Confluent Hypergeometric function, (3.14) becomes

$$
\begin{aligned}
\bar{P}_{D: M R C: N a k}=1- & \frac{A}{D} \frac{\Gamma(L m)}{\Gamma(m)} e^{-\frac{\lambda}{2}} \sum_{n=u}^{\infty} \sum_{k=0}^{\infty}\left(\frac{\lambda}{2}\right)^{\frac{n}{2}} \frac{\Gamma(m+k) C^{k}}{\Gamma(L m+k) k !} \\
& \times \underbrace{\int_{0}^{\infty} \gamma^{L m-1-\frac{n}{2}+k} e^{-\gamma(1+B)} I_{n}(\sqrt{2 \lambda \gamma}) \mathrm{d} \gamma}_{I_{A}} .
\end{aligned}
$$

To solve the integral $I_{A}$ in (3.15), we use [[77], Eq. (6.643/2)] given as

$$
\begin{array}{r}
\int_{0}^{\infty} x^{\mu-\frac{1}{2}} e^{-\alpha x} I_{2 \nu}(2 \beta \sqrt{x}) \mathrm{d} x=\frac{\Gamma\left(\mu+\nu+\frac{1}{2}\right)}{\Gamma(2 \nu+1)} \beta^{-1} e^{\frac{\beta^{2}}{2 \alpha}} \alpha^{-\mu} M_{-\mu, \nu}\left(\frac{\beta^{2}}{\alpha}\right) \\
{\left[\operatorname{Re}\left(\mu+\nu+\frac{1}{2}\right)>0\right]}
\end{array}
$$

where $M_{\mu, \nu}($.$) denotes the Whittaker function given by [[75], (13.1.32)] as$

$$
M_{\mu, \nu}(z)=z^{\nu+\frac{1}{2}} e^{-\frac{z}{2}}{ }_{1} F_{1}\left(\nu-\mu+\frac{1}{2} ; 1+2 \nu ; z\right) .
$$


Now, by choosing appropriate parameter values to satisfy the condition within, the solution of $(3.15)$ is $[78,79]$

$$
\begin{aligned}
\bar{P}_{D M R C: \text { onst }}=1-\frac{A}{D} \frac{\Gamma(L m)}{\Gamma(m)} e^{-\frac{\lambda}{2}} \sum_{n=u}^{\infty} & \sum_{k=0}^{\infty}\left(\frac{\lambda}{2}\right)^{n} \frac{\Gamma(m+k) C^{k}}{\Gamma(n+1)(1+B)^{L m+k} k !} \\
& \times{ }_{1} F_{1}\left(L m+k ; n+1 ; \frac{\lambda}{2(1+B)}\right) .
\end{aligned}
$$

Note that $\overline{P_{D}} M R C$ :Const expression in (3.18) is not restricted to integer $m$ or $u$ values. For i.i.d. diversity branches, (3.18) reduces to [[34], Eq. (24)]. The infinite series in (3.18) are upper bounded by the monotonically decreasing ${ }_{1} F_{1}(. ; . ;$.$) [80] for fixed$ values of $m, \rho, \lambda, \bar{\gamma}$, hence it converges rapidly. Consequently, the number of $N$-terms required for five digit accuracy could be found using this constraint as shown in Table 3.1 .

\subsubsection{Alternative Expression for $\overline{P_{D}} M R C:$ Const}

An alternative canonical Marcum Q-function representation where, $u$ is not restricted to positive integer values (i.e. $u \in\{0.5,1,1.5,2,2.5, \ldots\})$ was introduced in [71] as

$$
Q_{u}(\sqrt{2 \gamma}, \sqrt{\lambda})=\sum_{n=0}^{\infty} \frac{\gamma^{n} e^{-\gamma} \Gamma\left(u+n, \frac{\lambda}{2}\right)}{\Gamma(u+n) n !}
$$


We derive an alternative closed-form expression for the $\overline{P_{D}}$ RC:Const given in (3.18) by substituting (3.19) and (3.6) into (2.12) as

$$
{\overline{P_{D}}}_{M R C: \text { Const }}=\frac{A}{D} \sum_{n=0}^{\infty} \frac{\Gamma\left(u+n, \frac{\lambda}{2}\right)}{\Gamma(u+n) n !} \underbrace{\int_{0}^{\infty} \gamma^{n+L m-1} e^{-\gamma(B+1)}{ }_{1} F_{1}(m, L m ; C \gamma) \mathrm{d} \gamma}_{I_{A}}
$$

To solve the integral $I_{A}$ in (3.20), we use [[77], Eq. (7.621/4)], this can be written as

$$
\begin{array}{r}
\int_{0}^{\infty} e^{-s t} t^{b_{2}-1}{ }_{1} F_{1}\left(a_{2} ; d_{2} ; k t\right) \mathrm{d} t=\Gamma\left(b_{2}\right) s^{-b_{2}} F\left(a_{2}, b_{2} ; d_{2} ; k s^{-1}\right), \\
{[|s|>|k|], b_{2}>0, s>0}
\end{array}
$$

where $F\left(a_{3}, b_{3} ; c_{3} ; z\right)={ }_{2} F_{1}\left(a_{3}, b_{3} ; c_{3} ; z\right)$ denotes the Gaussian Hypergeometric function given in $([75],(15.1 .1))$. Then by choosing appropriate parameters values, we solve the expression in (3.20) to satisfy the condition within as $[78,79]$

$$
{\overline{P_{D}}}_{R R C \text { Const }}=\frac{A}{D} \sum_{n=0}^{\infty} \frac{\Gamma\left(u+n, \frac{\lambda}{2}\right) \Gamma(L m+n)}{\Gamma(u+n)(1+B)^{L m+n} n !}{ }_{2} F_{1}\left(m, L m+n ; L m ; \frac{C}{1+B}\right) \text {, }
$$

where $A, B, C$ and $D$ are given in equations (3.7), (3.8), (3.9) and (3.10) respectively. Note, both $\bar{P}_{D \text { :Const }}$ expressions in (3.18) and (3.22) are not restricted to integer values of $u$ and $m$. However, the expression in (3.22) is more desirable since it has only one infinite series term, hence, less complicated mathematically. 


\subsection{Exponential Correlation}

In this section, we will investigate exponentially $L$ correlated Nakagami- $m$ fading branches with MRC diversity. The PDF of the output SNR's for $L$ MRC Nakagami- $m$ branches with exponential correlation is given by [66] as

$$
p_{\gamma M R C: E x p}(\gamma)=\frac{1}{\Gamma\left(\frac{m L^{2}}{\delta}\right)\left(\frac{\delta \bar{\gamma}}{L m}\right)^{\frac{m L^{2}}{\delta}}} \gamma^{\frac{m L^{2}}{\delta}-1} \exp \left(-\frac{L m \gamma}{\delta \bar{\gamma}}\right)
$$

where:

$$
\delta=L+\frac{2 \rho}{1-\rho}\left(L-\frac{1-\rho^{L}}{1-\rho}\right), \quad 0 \leq \rho<1 .
$$

It is convenient to write $(3.23)$ as

$$
p_{\gamma M R C: \operatorname{Exp}}(\gamma)=\frac{1}{\Gamma(a) b^{a}} \gamma^{a-1} e^{-\frac{\gamma}{b}}
$$

where, $a$ and $b$ are given by (3.26) and (3.27) respectively,

$$
\begin{aligned}
& a=\frac{m L^{2}}{\delta}, \\
& b=\frac{\delta \bar{\gamma}}{L m} .
\end{aligned}
$$

The average detection probability is obtained by substituting (3.25) into (2.12). This yields

$$
\bar{P}_{D: M R C: E x p}=\frac{1}{\Gamma(a) b^{a}} \int_{0}^{\infty} Q_{u}(\sqrt{2 \gamma}, \sqrt{\lambda}) \gamma^{a-1} e^{-\frac{\gamma}{b}} \mathrm{~d} \gamma
$$


By changing the variable $x=\sqrt{2 \gamma}$ and with some manipulation, yields

$$
\bar{P}_{D: M R C: E x p}=\frac{1}{2^{a-1} \Gamma(a) b^{a}} \underbrace{\int_{0}^{\infty} Q_{u}(x, \sqrt{\lambda}) x^{2 a-1} e^{-\frac{x^{2}}{2 b}} \mathrm{~d} x}_{I} .
$$

Using Nuttall integrals and by satisfying the inner conditions by choosing appropriate parameter values as given in [[81], Eq. (29)], this can be written as

$$
\begin{aligned}
& \int_{0}^{\infty} Q_{u}(\alpha x, \beta) x^{q} e^{-\frac{p^{2} x^{2}}{2}} \mathrm{~d} x \equiv G_{u} \\
& =G_{u-1}+\frac{1}{2(u-1) !\left(\frac{p^{2}+\alpha^{2}}{2}\right)^{\frac{q+1}{2}}} \Gamma\left(\frac{q+1}{2}\right)\left(\frac{\beta^{2}}{2}\right)^{u-1} \\
& \times e^{-\frac{\beta^{2}}{2}}{ }_{1} F_{1}\left(\frac{q+1}{2} ; u ; \frac{\beta^{2}}{2} \frac{\alpha^{2}}{p^{2}+\alpha^{2}}\right), q>-1 .
\end{aligned}
$$

we can solve $I$ by evaluating $G_{u}$ recursively for $q>-1$ and restricted $u$ integer values as

$$
\begin{aligned}
& G_{u}=G_{u-1}+A_{u-1} F_{u} \\
& =G_{u-2}+A_{u-2} F_{u-2}+A_{u-1} F_{u-1} \\
& \vdots \\
& =G_{1}+\sum_{n=1}^{u-1} A_{n} F_{n+1},
\end{aligned}
$$

where $A_{n}$ and $F_{n}$ are given respectively by

$$
A_{n}=\frac{1}{2(n !)\left(\frac{p^{2}+\alpha^{2}}{2}\right)^{\frac{q+1}{2}}} \Gamma\left(\frac{q+1}{2}\right)\left(\frac{\beta^{2}}{2}\right)^{n} e^{-\frac{\beta^{2}}{2}},
$$




$$
F_{n}={ }_{1} F_{1}\left(\frac{q+1}{2} ; n ; \frac{\beta^{2}}{2} \frac{\alpha^{2}}{p^{2}+\alpha^{2}}\right) .
$$

Hence, we can solve $\bar{P}_{D: M R C: E x p}$ in $(3.29)$ as $[78,79]$

$$
\bar{P}_{D: M R C: E x p}=\zeta\left[G_{1}+\frac{\eta}{2} \sum_{n=1}^{u-1}\left(\frac{\lambda}{2}\right)^{n} \frac{1}{n !}{ }_{1} F_{1}\left(a ; n+1 ; \frac{\lambda b}{2(1+b)}\right)\right]
$$

where, $\zeta$ and $\eta$ are given, respectively, by

$$
\begin{aligned}
\zeta & =\frac{1}{2^{a-1} \Gamma(a) b^{a}} \\
\eta & =\Gamma(a)\left(\frac{2 b}{1+b}\right)^{a} e^{-\frac{\lambda}{2}}
\end{aligned}
$$

Here, $a$ and $b$ are given in (3.26) and (3.27) respectively. Then we can obtain $G_{1}$ by evaluating the following integral containing the first order of Marcum $Q$-function $Q(.,$.$) as$

$$
G_{1}=\int_{0}^{\infty} Q(x, \sqrt{\lambda}) x^{2 a-1} e^{-\frac{x^{2}}{2 b}} \mathrm{~d} x
$$

Using [[81], Eq. (25)], we evaluate $G_{1}$ for integer values of $(a)$ as $[78,79]$

$$
\begin{aligned}
& G_{1}=\frac{2^{a-1}(a-1) !}{\left(\frac{1}{b}\right)^{2 a}}\left(\frac{b}{1+b}\right) e^{-\frac{\lambda}{2(1+b)}}\left[\left(1+\frac{1}{b}\right)\left(\frac{1}{1+b}\right)^{a-1} L_{a-1}\left(-\frac{\lambda b}{2(1+b)}\right)\right. \\
& \left.+\sum_{n=0}^{a-2}\left(\frac{1}{1+b}\right)^{n} L_{n}\left(-\frac{\lambda b}{2(1+b)}\right)\right]
\end{aligned}
$$

Here, $L_{n}($.$) denotes Laguerre polynomial of n$-degree [77]. Please note, without diversity $(L=1)$, the correlation is zero $(\rho=0)$, then (3.34) and (3.38) reduce to, (20) and (23) in [23] respectively, serving as a proof. 


\subsubsection{Alternative $\bar{P}_{D: M R C: E x p}$ Simple Expression}

The expression in (3.34) is restricted to integer $u$ and $(a)$ values. However, we included that in our derivation to show that it reduces to the corresponding expression of a single antenna receiver derived in [23]. We can also derive a more general expression for $\bar{P}_{D: M R C: E x p}$ by inserting (3.12) in (3.28) and by using the definition of the PDF in (3.13) with some simplifications. This yields

$$
\bar{P}_{D: M R C: E x p}=1-\frac{1}{\Gamma(a) b^{a}} e^{-\frac{\lambda}{2}} \sum_{n=u}^{\infty}\left(\frac{\lambda}{2}\right)^{\frac{n}{2}} \int_{0}^{\infty} \gamma^{a-\frac{n}{2}-1} e^{-\gamma\left(\frac{b+1}{b}\right)} I_{n}(\sqrt{2 \lambda \gamma}) \mathrm{d} \gamma
$$

Following the same procedures as in (3.18), using (3.16) and (3.17) while choosing appropriate parameters values to satisfy the conditions, we can show $[78,79]$

$$
\bar{P}_{D: M R C: E x p}=1-\frac{1}{(1+b)^{a}} e^{-\frac{\lambda}{2}} \sum_{n=u}^{\infty}\left(\frac{\lambda}{2}\right)^{\frac{n}{2}} \frac{1}{\Gamma(n+1)}{ }_{1} F_{1}\left(a ; n+1 ; \frac{\lambda b}{2(1+b)}\right),
$$

where, $a$ and $b$ are given by (3.26) and (3.27), respectively.

Clearly, (3.40) is less complicated mathematically than (3.34) since it does not contain the first order of Marcum $Q$-function and both $m$ and $u$ are not restricted to integer values. For i.i.d $L$-fading branches $(\rho=0),(3.40)$ reduces to [[34], Eq. (24)].

Again, we can easily show the rapid convergence of the infinite series in (3.40) is upper bounded by the monotonically decreasing ${ }_{1} F_{1}\left(. ; ;\right.$; .) since both $a$ and $\frac{\lambda b}{2(1+b)}$ are constants for fixed $m, \rho, \lambda, \bar{\gamma}$ and $L$ values. Therefore, the number of $N$-terms required for five digit accuracy could be found using this constraint as shown in Table 3.1. 
Table 3.1: Terms required for five digits accuracy

\begin{tabular}{|l|l|}
\hline $\bar{P}_{D: \text { Const }}:$ & $\tilde{E}_{N} \mid, u=2, \bar{\gamma}=20 \mathrm{~dB}, \mathrm{~m} \in\{1,2,3,4\}$ and $\mathrm{L} \in\{2,3,4\}$ \\
\hline$\rho$ & $N_{n}, N_{k}$ \\
\hline 0 & 10,1 \\
\hline $0.2,0.4,0.6,0.8$ & 10,5 \\
\hline $\bar{P}_{D: \text { Exp }}$ & $\tilde{E}_{N} \mid, u=2, \bar{\gamma}=20 \mathrm{~dB}, \mathrm{~m} \in\{1,2,3,4\}$ and $\mathrm{L} \in\{2,3,4\}$ \\
\hline$\rho$ & $N_{n}$ \\
\hline $0,0.2,0.4,0.6,0.8$ & 5 \\
\hline
\end{tabular}

\subsubsection{Arbitrary Real $u$-values Alternative $\bar{P}_{D: M R C: E x p}$ Expression}

Expression in (3.34) is restricted to integer $u$ values. Recently, for arbitrary real $u$-values, an analytic solution in terms of a closed-form expression for the integral in (3.29) was introduced in [82] as

$$
\int_{0}^{\infty} Q_{u}(\alpha x, \beta) x^{2 k-1} e^{-p x^{2}} \mathrm{~d} x=\sum_{n=0}^{\infty} \frac{\alpha^{2 n} 2^{k} \Gamma(k+n) \Gamma\left(u+n, \frac{\beta^{2}}{2}\right)}{\Gamma(u+n)\left(\alpha^{2}+2 p\right)^{k+n} n !} .
$$

Hence, by using (3.41) we can express the $\bar{P}_{D: M R C: E x p}$ in $(3.29)$ as $[78,79]$

$$
\bar{P}_{D: M R C: E x p}=\frac{2}{\Gamma(a) b^{a}} \sum_{n=0}^{\infty} \frac{\Gamma(a+n) \Gamma\left(u+n, \frac{\lambda}{2}\right)}{\Gamma(u+n)\left(1+\frac{1}{b}\right)^{a+n} n !},
$$

where, $a$ and $b$ are given in (3.26) and (3.27), respectively. Interestingly, (3.42) is less complicated mathematically than both (3.34) and (3.40) since it does not contain first order of Marcum $Q$-function or hypergeometric function. 


\subsection{Performance Analysis}

In this section, we verify the derived closed-form expressions for different cases of interest for spectrum sensing models with MRC diversity technique. The impact of multipath fading and correlation on average detection probability $\bar{P}_{D}$ (as a performance factor) is analyzed and investigated. To this end, we produce the corresponding CROC graphs, $\left(P_{D m}\right.$ versus $\left.P_{F}\right)$ for MRC diversity technique in correlated Nakagami- $m$ fading channel as follows.

We calculate the threshold $\lambda$ for $u=2, \bar{\gamma}=20 \mathrm{~dB}, m \in\{1,2,3,4\}$ and, $\rho \in$ $\{0,0.2,0.4,0.6,0.8\}$ for different values of $P_{F}$ with the aid of (2.10). It's worthwhile to mention that we produced figures for two $m$ values, $m \in\{1,4\}$, in order to reduce the number of figures to the minimum, however, our derived expressions can be evaluated for any corresponding values.

In Figure. 3.2, numerical results obtained from Monte-Carlo simulation almost match that derived closed-form expressions, therefore, this validates the derived expressions.

Figures 3.3 and 3.4 depict the detection performance for constant and exponential correlations respectively, for MRC with $L$-correlated Nakagami- $m$ fading branches using the derived closed-form expressions in Section 3.2 and Section 3.3. Comparing each graph in Figure 3.3a with its corresponding one in $3.3 \mathrm{~b}$, we notice that the detection probability $P_{D}$ increases (equivalently missed-detection probability $P_{D m}$ decreases) as the fading parameter $m$ increases (low fading), hence, improving the detection performance. This is as expected. Similar results could be inferred when a comparison of the corresponding graphs is done between Figures 3.4a and 3.4b in exponentially correlated branches. However, for each value of $\rho$ in Figures 3.3 and 3.4, 


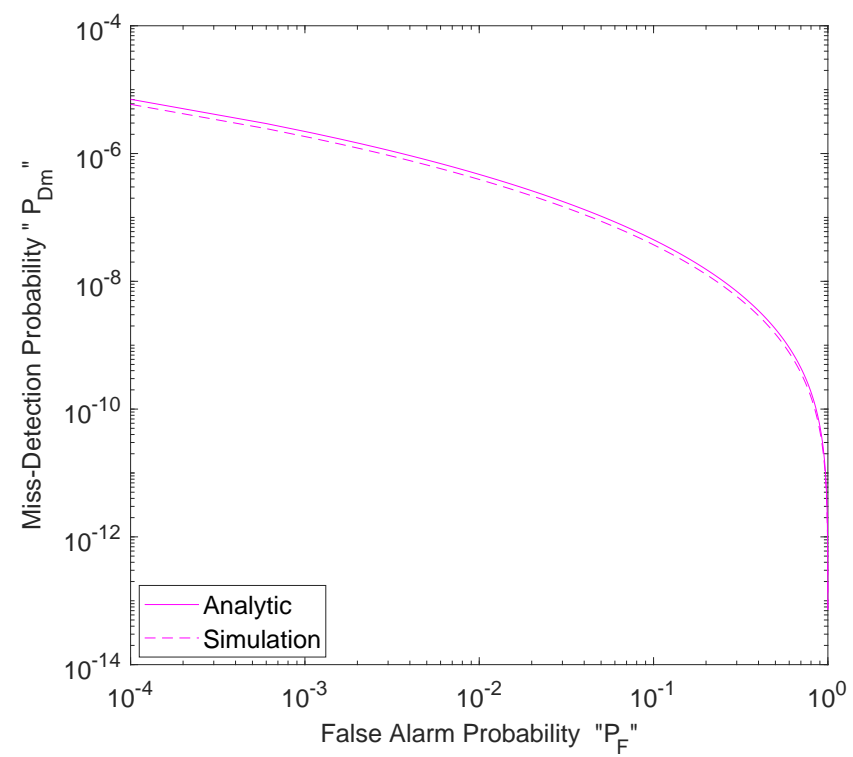

Figure 3.2: Analytic (solid) versus simulation (dashed) results for MRC with $L=2$, $m=4$ and $\rho=0.8$.

one can notice clearly the degradation in detection probability due to the increment in correlation values among diversity branches. However, as the number of diversity branches $L$ increases, correlation impact is compensated for, resulting in improved detection probability. Note, a difference in the performance of the two investigated correlation models is observed. We observe that constant correlation model shows minor performance degradation in terms of decreasing $P_{D}$ compared to exponential correlation model. In fact, performance difference is more pronounced for high $L, m$ and $\rho$ values. We observe that the separation among the adjacent curves for each case in Figures 3.3 and 3.4 increases as $L$ increases indicating that achieving the same $P_{D}$ values for different $L$ will increase the corresponding $P_{F}$ values, which we try to keep the latter as low as possible (IEEE 802.22 standard specifications require $P_{F} \leq 0.1$ ). However, this is not the case for the same detection scenario if the uncorrelated 


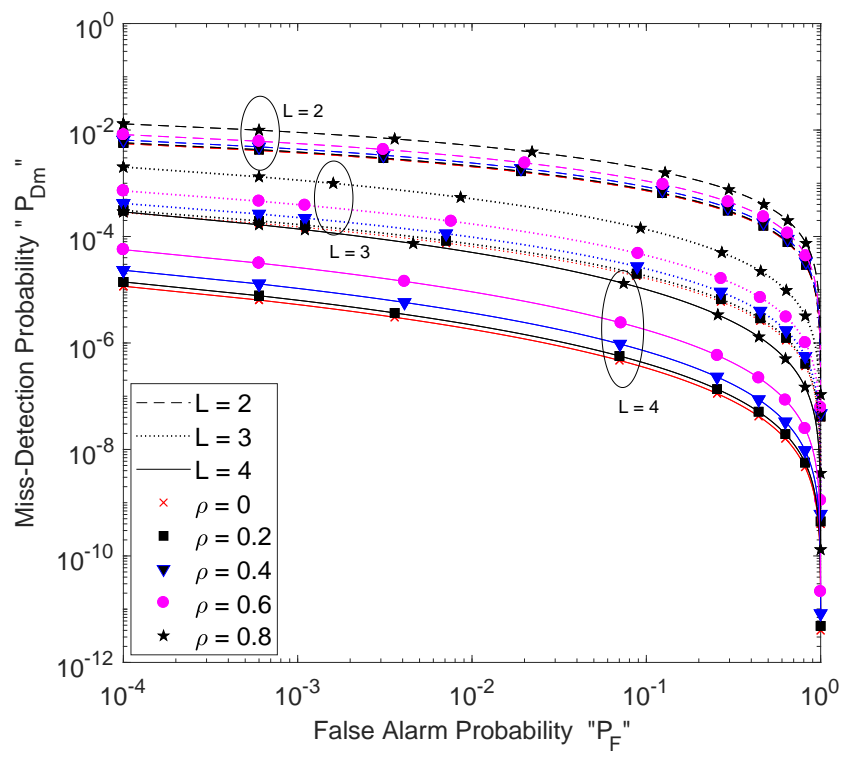

(a) $\quad m=1$ (Rayleigh: deep fading)

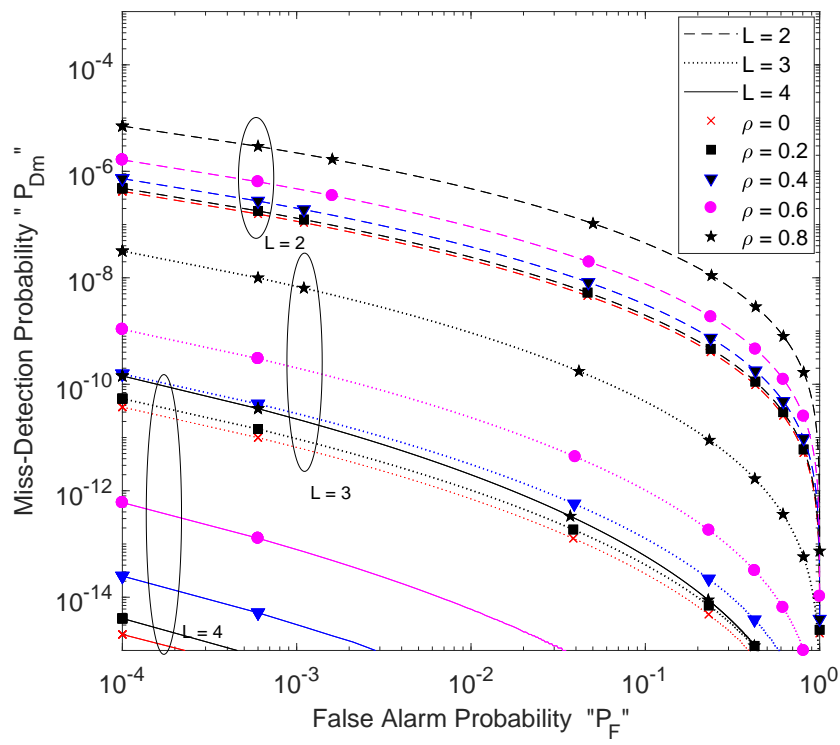

(b) $\quad m=4$ (Low fading)

Figure 3.3: Constant correlated Nakagami- $m$ branches with MRC diversity; $\bar{\gamma}=20$ $\mathrm{dB}, L=2,3$ and 4 for different $\rho$ values. 


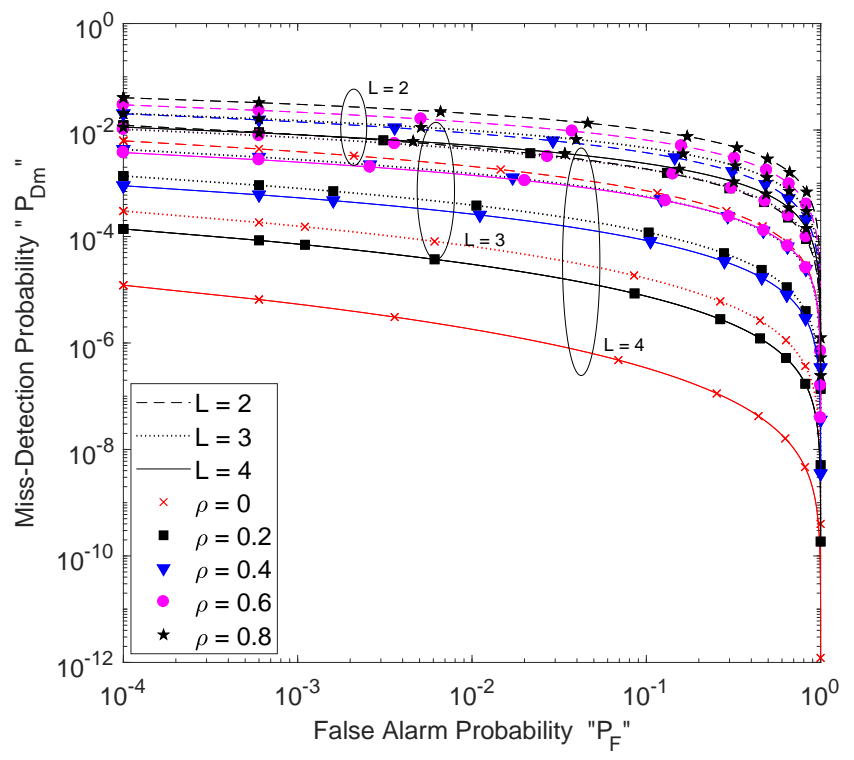

(a) $m=1$ (Rayleigh: deep fading)

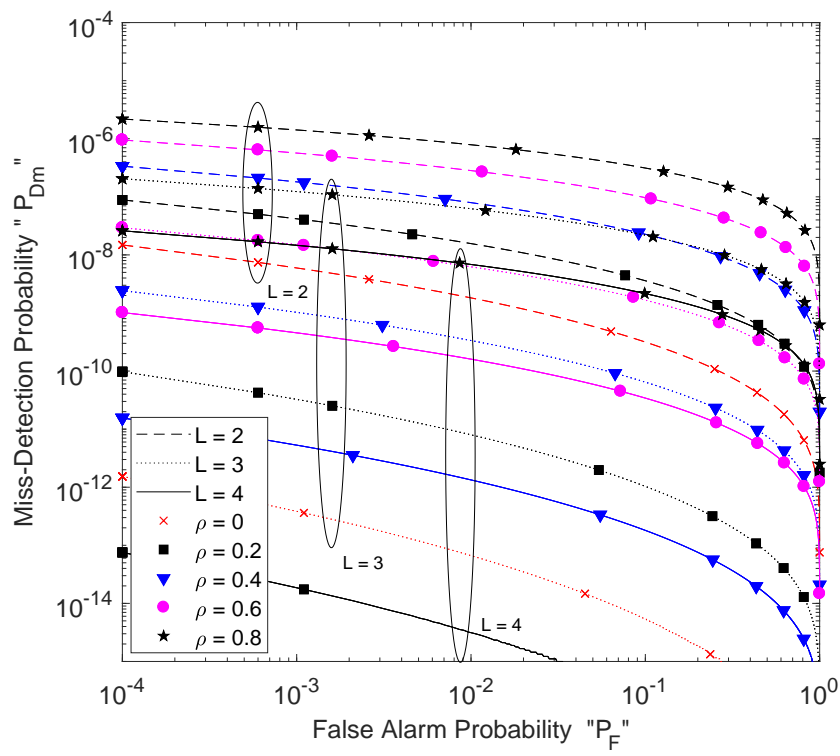

(b) $\quad m=4$ (Low fading)

Figure 3.4: Exponentially correlated Nakagami- $m$ branches with MRC diversity; $\bar{\gamma}=20 \mathrm{~dB}, L=2,3$ and 4 for different $\rho$ values. 
diversity fading branches are assumed. Figure 3.5 shows this behavior ${ }^{1}$. Therefore, we say that constant correlation model shows minor performance degradation in terms of decreasing $P_{D}$ compared to exponential correlation model. In fact, performance difference is more pronounced for high $L, m$ and $\rho$ values. In other word, MRC with constant correlation model is clearly less affected by correlation when it is low and also, to some extent, by high correlation environment compared with exponential correlation model. Consequently, MRC with constant correlation results in relatively higher throughput compared with corresponding exponential model.

\footnotetext{
${ }^{1}$ In order to visualize clearly the behavior difference between the two correlation models, we connected the corresponding points of each case in $3.5 \mathrm{a}$ and $3.5 \mathrm{~b}$ with straight lines as in $3.5 \mathrm{c}$ and $3.5 \mathrm{~d}$.
} 


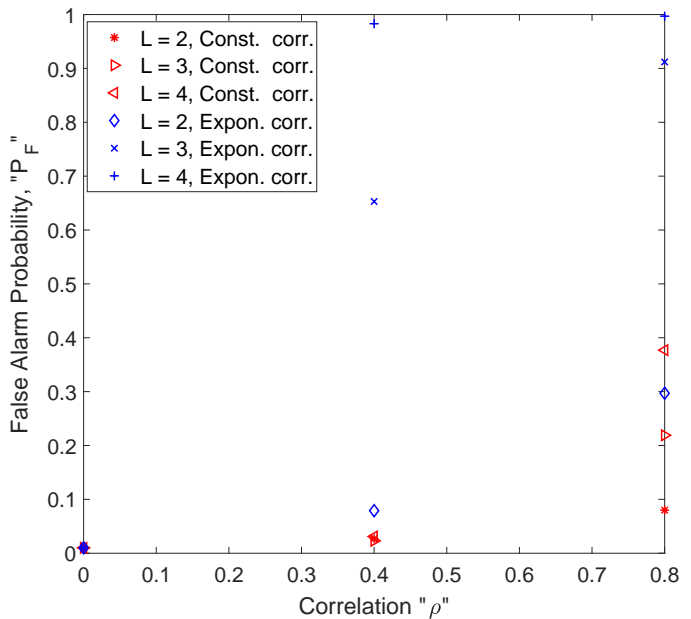

(a) $m=1$ (Rayleigh)

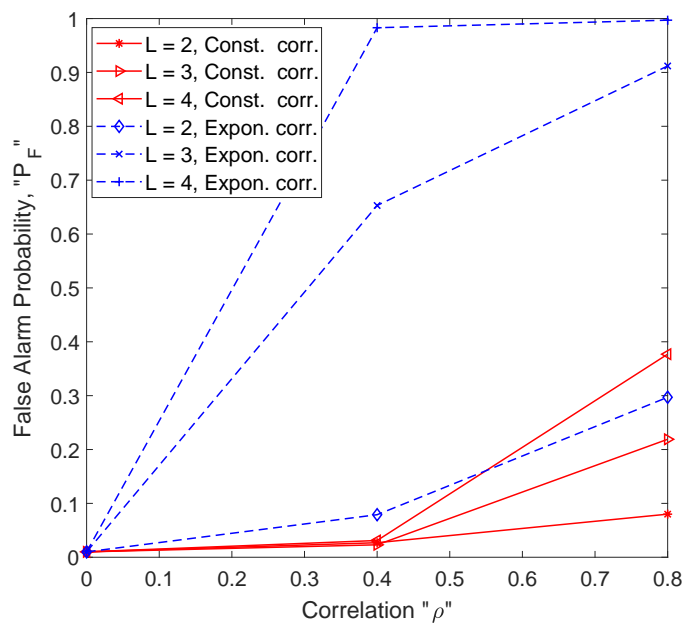

(c) $m=1$ (Rayleigh)

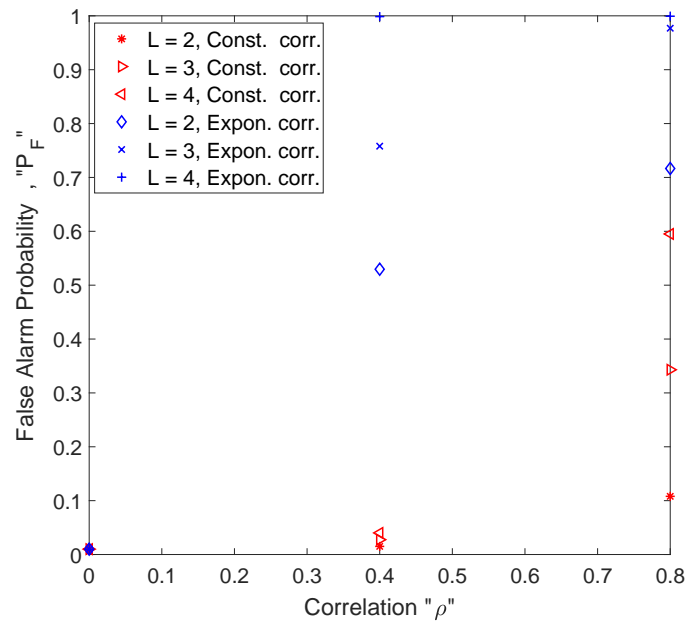

(b) $m=2$

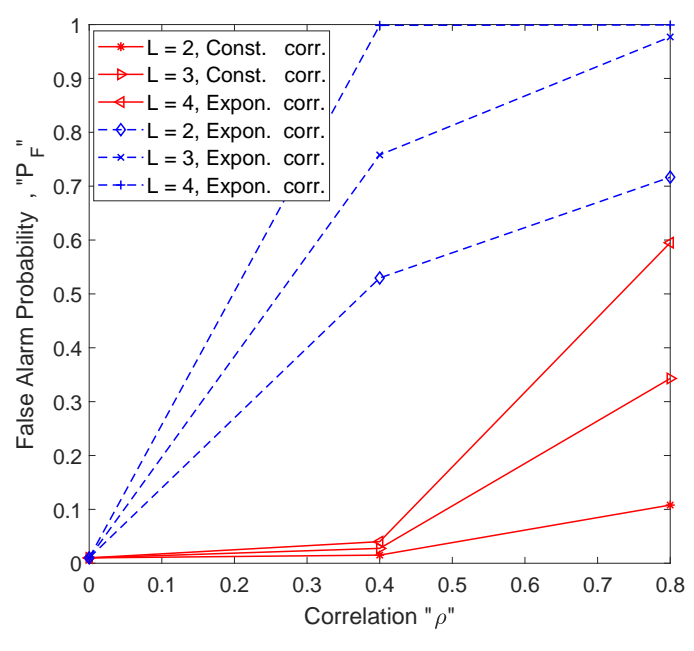

(d) $m=2$

Figure 3.5: Correlation impact on probability of false alarm in correlated Nakagami- $m$ branches with MRC diversity; $\bar{\gamma}=20 \mathrm{~dB}, L=2,3$ and 4 for different $\rho$ values, (a) and (b) represent the exact points while in $(\mathrm{c})$ and $(\mathrm{d})$, corresponding points are connected with straight lines for better visualization of the performance difference. Constant correlation (solid), exponential correlation (dashed). 


\section{Chapter 4}

\section{Dual Correlated Nakagami- $m$ \\ Branches with EGC Diversity}

In this chapter, we consider a detection scenario when EGC diversity technique is employed with dual correlated Nakagami- $m$ fading branches. We derive an exact closed-form expression for the average detection probability of each sensing scenario. We do performance analysis using CROC graphs to evaluate the derived expressions by studying the fading and correlation impacts on the detection probability.

\subsection{EGC Diversity}

Equal Gain Combining is a suboptimal, reduced complexity and limited with coherent detection technique. Unlike MRC, EGC weights each branch equally before combining, 


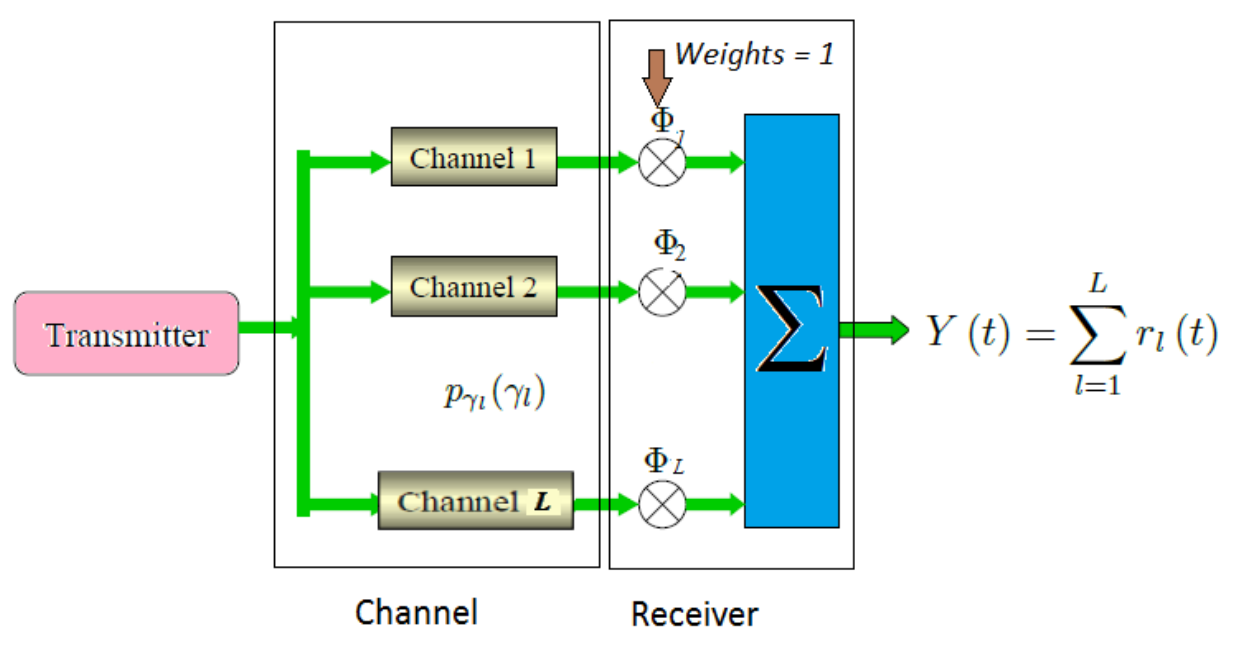

Figure 4.1: Equal Gain Combining (EGC).

then cophased and summed as shown in Figure $4.1^{1}$, resulting in a new combined signal

$$
Y(t)=\sum_{l=1}^{L} r_{l}(t)
$$

where $r_{l}$ is the received signal of $l^{\text {th }}$ branch given in (2.1). Therefore, EGC doesn't necessitate channel amplitude estimation for each branch. The EGC's conditional SNR per symbol is given as [[19] Eq. (9.51)]

$$
\gamma_{E G C}=\frac{\left(\sum_{l=1}^{L} \alpha_{l}\right)^{2} E_{s}}{\sum_{i=l}^{L} N_{l}}
$$

where $\alpha_{l}$ denotes the fading envelope amplitude which is a random variable that controls the SNR.

\footnotetext{
${ }^{1}$ EGC combiner diagram is similar to that for MRC as in Figure3.1 with only the exception mentioned above.
} 


\section{$4.2 \quad \bar{P}_{D: E G C, 2}$ Expression}

In this section, we will investigate arbitrarily dual correlated Nakagami- $m$ fading branches with EGC diversity.

For dual identical and arbitrarily correlated Nakagami- $m$ fading channels with EGC combiner, the PDF of the output SNR could be obtained by changing variables in [ [83], Eq. (1)] yields

$$
\begin{aligned}
p_{\gamma E G C}(\gamma) & =\frac{(1-\rho)^{m}}{\Gamma(m)} \sum_{k=0}^{\infty} \frac{B\left(2(m+k), \frac{1}{2}\right) c^{2(m+k)} \rho^{k}}{k ! \Gamma(m+k) 2^{2(m+k-1)}} \\
& \times \gamma^{2(m+k)-1} e^{-2 c \gamma}{ }_{1} F_{1}\left(2(m+k) ; 2(m+k)+\frac{1}{2} ; c \gamma\right), \gamma \geq 0
\end{aligned}
$$

where, $c=\frac{m}{\bar{\gamma}(1-\rho)}$ and $B(\alpha, \beta)=\frac{\Gamma(\alpha) \Gamma(\beta)}{\Gamma(\alpha+\beta)}$ denotes Beta function and ${ }_{1} F_{1}(., . ;$.$) as$ defined in (3.5).

We derive the average detection probability by substituting (4.3) into (2.12) and using (3.5), (3.12) and (3.13) with some simplification yields

$$
\begin{aligned}
\bar{P}_{D: E G C, 2} & =1-\frac{(1-\rho)^{m}}{\Gamma(m)} \Gamma\left(\frac{1}{2}\right) e^{\frac{-\lambda}{2}} \sum_{n=u}^{\infty} \sum_{k=0}^{\infty} \sum_{i=0}^{\infty}\left(\frac{\lambda}{2}\right)^{\frac{n}{2}} \\
& \times \frac{\Gamma(2(m+k)+i) c^{2(m+k)+i} \rho^{k}}{\Gamma(m+k) \Gamma\left(2(m+k)+i+\frac{1}{2}\right) 2^{2(m+k-1)} k ! i !} \\
& \times \underbrace{\int_{0}^{\infty} \gamma^{2(m+k)+i-\frac{n}{2}-1} e^{-\gamma(2 c+1)} I_{n}(\sqrt{2 \lambda \gamma}) \mathrm{d} \gamma}_{I} .
\end{aligned}
$$

Following the same procedures as in (3.18), using (3.16), then choosing appropriate parameters' values to satisfy the condition in order to solve the integral $I$, then using 
(3.17) we can further simplify this expression. Note, here $u$ and $m$ are not-restricted to integer values.

Using mathematical manipulation, we derive the final expression for $\bar{P}_{D: E G C, 2}$ as [79]

$$
\begin{aligned}
\bar{P}_{D: E G C, 2}= & -\frac{4 \sqrt{\pi}(1-\rho)^{m}}{2^{2 m} \Gamma(m)} e^{-\frac{\lambda}{2}} \sum_{n=u}^{\infty} \sum_{k=0}^{\infty} \sum_{i=0}^{\infty}\left(\frac{\lambda}{2}\right)^{n} \\
\times & \frac{\Gamma^{2}(2(m+k)+i) c^{2(m+k)+i} \rho^{k}}{\Gamma(m+k) \Gamma\left(2(m+k)+i+\frac{1}{2}\right)(2 c+1)^{2(m+k)+i} 2^{2 k} n ! k ! i !} \\
& \quad \times{ }_{1} F_{1}\left(2(m+k)+i+1 ; 1+n ; \frac{\lambda}{2(2 c+1)}\right) .
\end{aligned}
$$

Note that, for i.i.d branches, (4.5) reduces to [[34], Eq. (35)].

Like earlier, the infinite series in (4.5) is upper bounded by the monotonically decreasing Confluent Hypergeometric function in $i, k$ and $n$ for given values of $m, \lambda, \bar{\gamma}$ and $\rho$. Hence, the number of $N$-terms required for five digit accuracy could be found using this constraint as shown in Table 4.1.

\subsection{Alternative $\bar{P}_{D: E G C, 2}$ Expression}

In this section, we derive an alternative more simple expression for the average detection probability. Using (3.19) and substituting (4.3) into (2.12) yields

$$
\begin{gathered}
\bar{P}_{D: E G C, 2}=\frac{(1-\rho)^{m}}{\Gamma(m)} \sum_{n=0}^{\infty} \sum_{k=0}^{\infty} \frac{\Gamma\left(u+n, \frac{\lambda}{2}\right)}{\Gamma(u+n)} \frac{B\left(2(m+k), \frac{1}{2}\right) c^{2(m+k)} \rho^{k}}{\Gamma(m+k) 2^{2(m+k-1)} n ! k !} \\
\quad \times \int_{0}^{\infty} \gamma^{2(m+k)+n-1} e^{-\gamma(2 c+1)}{ }_{1} F_{1}\left(2(m+k) ; 2(m+k)+\frac{1}{2} ; c \gamma\right) \mathrm{d} \gamma
\end{gathered}
$$


Table 4.1: Terms required for five digits accuracy

\begin{tabular}{|l|l|l|l|l|l|}
\hline \multicolumn{2}{|c|}{$\bar{P}_{D, E G C, 2}:$} & $\tilde{E}_{N}$ & \multicolumn{3}{|c|}{$, u=2, P_{F}=0.01, \bar{\gamma}=20 \mathrm{~dB}$} \\
\hline$\rho$ & $m=1$ & $m=2$ & $m=3$ & $m=4$ \\
& $N_{n}, N_{k}, N_{i}$ & $N_{n}, N_{k}, N_{i}$ & $N_{n}, N_{k}, N_{i}$ & $N_{n}, N_{k}, N_{i}$ \\
\hline 0 & $13,1,9$ & $10,1,9$ & $5,1,9$ & $3,1,9$ \\
\hline 0.2 & $12,3,9$ & $11,3,9$ & $8,3,9$ & $5,3,9$ \\
\hline 0.4 & $12,3,9$ & $10,3,9$ & $5,3,9$ & $3,3,9$ \\
\hline 0.6 & $15,3,9$ & $10,3,9$ & $4,3,9$ & $3,3,9$ \\
\hline 0.8 & $18,3,9$ & $8,3,9$ & $3,3,9$ & $2,3,9$ \\
\hline
\end{tabular}

Following the same procedure as in (3.22) by using (3.21) and satisfying the condition therein, the average detection probability as [79]

$$
\begin{aligned}
\bar{P}_{D: E G C, 2}= & \frac{4 \sqrt{\pi}(1-\rho)^{m}}{2^{m} \Gamma(m)} \sum_{n=0}^{\infty} \sum_{k=0}^{\infty} \frac{\Gamma\left(u+n, \frac{\lambda}{2}\right)}{\Gamma(u+n)} \frac{\Gamma(2(m+k)) c^{2(m+k)} \rho^{k}}{\Gamma(m+k) 2^{2 k} n ! k !} \\
& \times \frac{\Gamma(2(m+k)+n)}{\Gamma\left(2(m+k)+\frac{1}{2}\right)(2 c+1)^{2(m+k)+n}} \\
& \times{ }_{2} F_{1}\left(2(m+k) ; 2(m+k)+n ; 2(m+k)+\frac{1}{2} ; \frac{c}{2 c+1}\right),
\end{aligned}
$$

where ${ }_{2} F_{1}\left(a_{2} ; b_{2} ; c_{2} ; z\right)$ denotes the Gaussian Hypergeometric function given in ([75], $(15.1 .1))$

\subsection{Performance Analysis}

In this section, we verify the derived closed-form expressions for different cases of interest for spectrum sensing model with EGC technique. The impact of multipath fading and correlation on average detection probability $\bar{P}_{D}$ (as a performance factor) is analysed using CROC. 


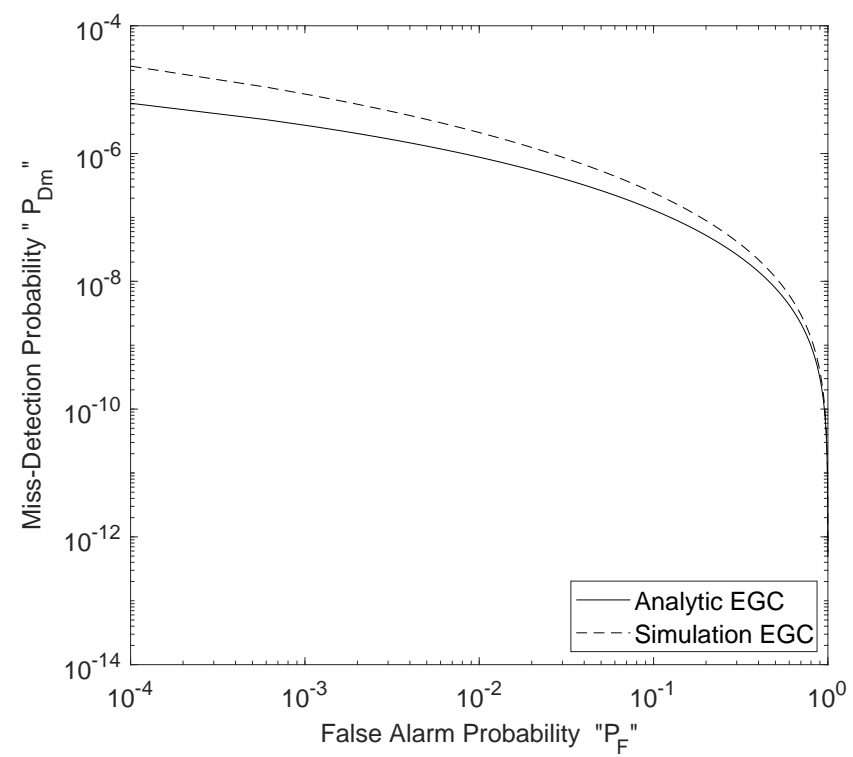

Figure 4.2: Analytic (solid) versus simulation (dashed) results for EGC with $L=2$, $m=4$ and $\rho=0.8$.

We follow the same procedures as we did in Sec. 3.4 to calculate the corresponding threshold for the assumed detection parameters to produce corresponding EGC' CROC graphs. In Figure. 4.2, numerical results obtained from Monte-Carlo simulation almost match that derived closed-form expressions. Reader may observe only a small difference $(\approx 0.03)$ between analytical and simulation curves for very low $P_{F}$ values. This is due to the inaccuracy arising from rounding off the infinite series. This validates the derived expressions.

Figure 4.3 shows the CROC graphs for dual correlated Nakagami- $m$ fading branches with EGC diversity for $m \in(1,4)$ and $\rho \in(0-0.8)$ values. For $m=1$, examining Figure 4.3a, one can clearly observe the deterioration in the cognitive detector performance $\left(P_{D m}\right.$ increases $)$ with the increment in the correlation coefficient $\rho$ values.

Similar results could be observed for $m=4$ in Figure 4.3b. However, as $m$ increases 


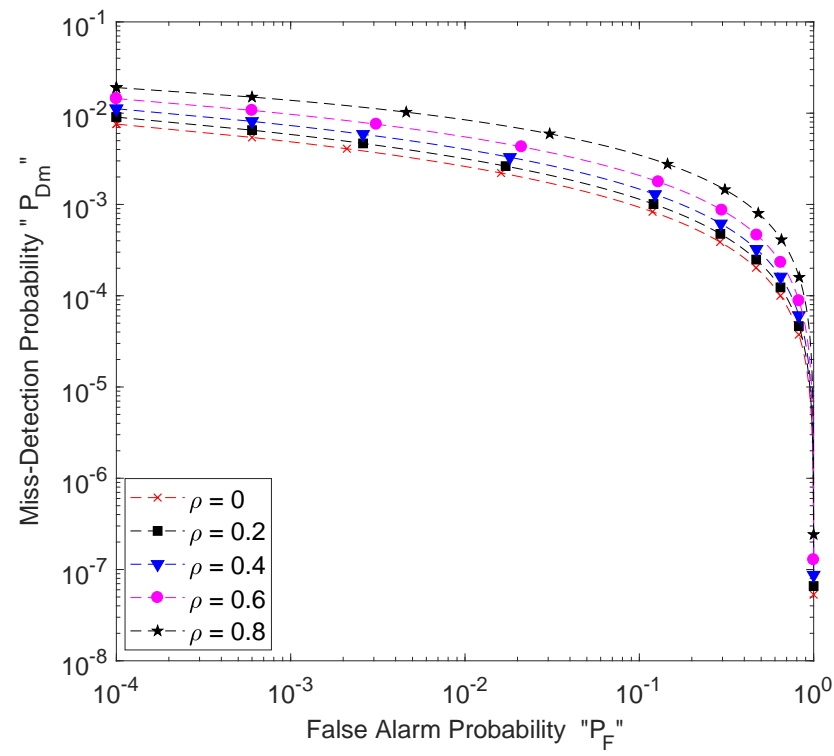

(a) $\quad m=1$ (Rayleigh: deep fading)

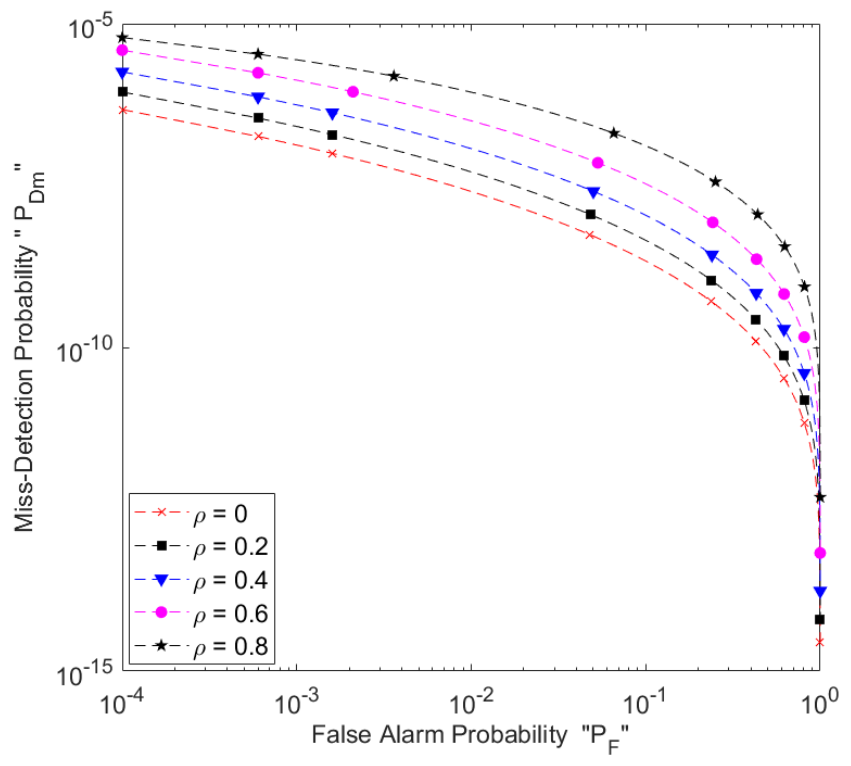

(b) $m=4$ (Low fading)

Figure 4.3: Arbitrarily correlated Nakagami- $m$ branches with EGC diversity: $L=2, \bar{\gamma}=20 \mathrm{~dB}$ for different $\rho$ values. 
(low fading) as in Figure 4.3b, detection performance improves in spite of the correlation impact.

Examining both above figures carefully, one can observe an interesting joint impact of both correlation and fading on detection. For deep fading (e.g. $m=1)$, the correlation is less adverse. As $m$ increase, correlation is relatively more adverse. However, the detector performance is much better since the detection probability is much higher than that for deep fading. Therefore, we say that for deep fading environment, correlation must be considered for accurate cognitive detection, while for low fading environment its effect can be ignored. 


\section{Chapter 5}

\section{Correlated Nakagami- $m$ Branches with SC Diversity}

In this chapter, we consider a detection scenario when SC diversity technique is employed for dual and triple diversity branches. For triple case, an exponentially correlated branches are considered. We derive the average detection probability for dual and triple Nakagami- $m$ correlated fading branches with SC diversity. We do performance analysis using CROC graphs to evaluate the derived expressions by studying the correlation impact on the detection probability. 


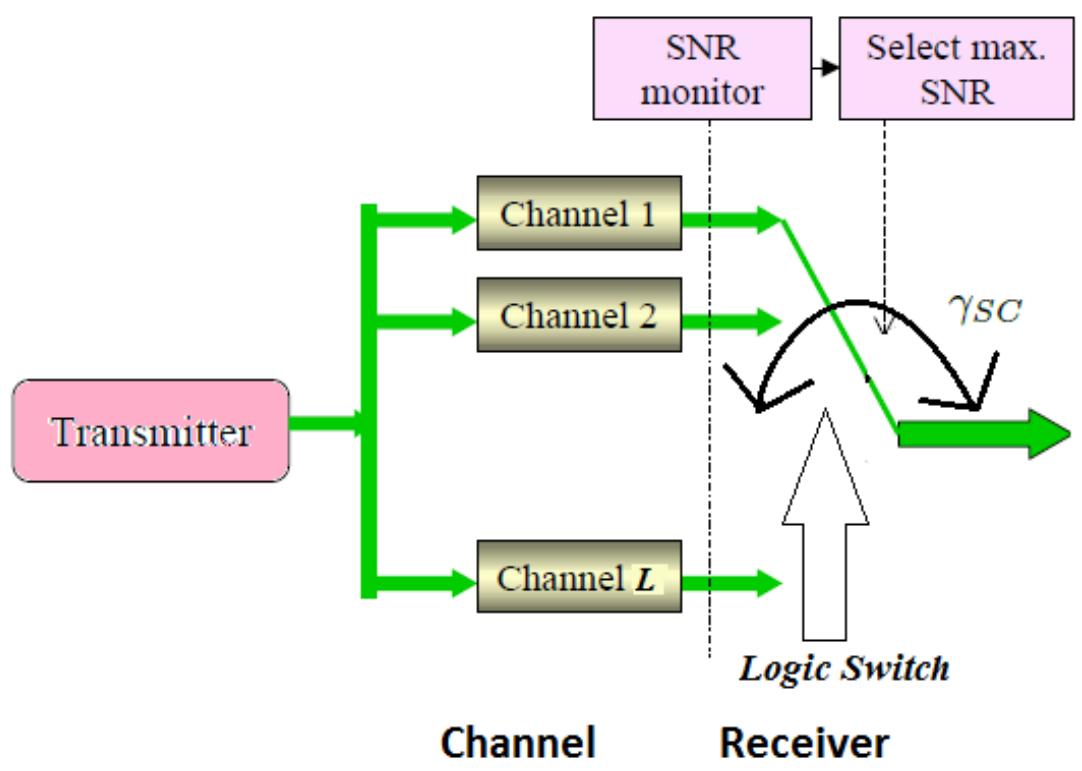

Figure 5.1: Selection Combining Technique (SC).

\subsection{SC Diversity}

Basically, the concept of SC is to select the best (largest magnitude) signal among all the signals received from different branches at the receiving end

$$
r=\max \left\{r_{l}, l=1,2, \ldots L\right\}
$$

Unlike MRC diversity technique where full knowledge of CSI is required, SC processes one branch. Therefore, it is less complicated diversity technique than both MRC and EGC, since the receiver selects just one branch at a time, namely the branch with the highest channel envelope value as shown in Figure 5.1. Moreover, SC can be used for both coherent and noncoherent modulation since no need for signal phase 
on each branch. However, SC is impractical for continuous transmission system since continuous monitoring is necessary to pick up the branch with highest SNR [19][84].

\subsection{Dual Arbitrarily Correlated Branches}

In this section, we will investigate $\mathrm{SC}$ with dual arbitrarily correlated Nakagami- $m$ fading branches. Using [[85], Eq. (20)] by assuming identical diversity branches and introducing changing variable with some mathematical simplification, the PDF of the output SNR for dual correlated Nakagami-m fading channels with SC combiner can be obtained as

$p_{\gamma S C}(\gamma)=\frac{2}{\Gamma(m)}\left(\frac{m}{\bar{\gamma}}\right)^{m} \gamma^{m-1} \exp \left(\frac{-m \gamma}{\bar{\gamma}}\right)\left[1-Q_{m}(\sqrt{2 a \rho \gamma}, \sqrt{2 a \gamma})\right], \quad \gamma \geq 0$

where $a=\frac{m}{\bar{\gamma}(1-\rho)}$ and $\rho$, denotes the correlation coefficient between the two fading envelopes. 


\subsubsection{Average Detection Probability}

We derive the average detection probability for dual correlated SC's diversity branches by substituting (5.2) into (2.12) and rearranging yields

$$
\begin{gathered}
\bar{P}_{D, S C, 2}=\frac{2}{\Gamma(m)}\left(\frac{m}{\bar{\gamma}}\right)^{m}[\underbrace{\int_{0}^{\infty} Q_{u}(\sqrt{2 \gamma}, \sqrt{\lambda}) \gamma^{m-1} \exp \left(-\frac{m \gamma}{\bar{\gamma}}\right) \mathrm{d} \gamma}_{I_{A}} \\
-\underbrace{\int_{0}^{\infty} Q_{u}(\sqrt{2 \gamma}, \sqrt{\lambda}) \gamma^{m-1} \exp \left(-\frac{m \gamma}{\bar{\gamma}}\right) Q_{m}(\sqrt{2 a \rho \gamma}, \sqrt{2 a \gamma}) \mathrm{d} \gamma}_{I_{B}}]
\end{gathered}
$$

Note this lengthy expression consists of two integrals, $I_{A}$ and $I_{B}$. We solve them separately.

\section{Evaluation of Integral $I_{A}$}

Let $x=\sqrt{2 \gamma}$, by changing variables, $\gamma=\frac{x^{2}}{2}$, and $d \gamma=x d x$, hence $I_{A}$ in (5.3) becomes

$$
I_{A}=\frac{1}{2^{m-1}} \underbrace{\int_{0}^{\infty} Q_{u}(x, \sqrt{\lambda}) x^{2 m-1} \exp \left(-\frac{m x^{2}}{2 \bar{\gamma}}\right) \mathrm{d} x}_{I}
$$


Using (29) given in Nuttall integrals [81] as

$$
\begin{aligned}
& \int_{0}^{\infty} Q_{u}(\alpha x, \beta) x^{q} e^{-\frac{p^{2} x^{2}}{2}} \mathrm{~d} x \equiv G_{u} \\
& =G_{u-1}+\frac{\Gamma\left(\frac{q+1}{2}\right)\left(\frac{\beta^{2}}{2}\right)^{u-1} e^{-\frac{\beta^{2}}{2}}}{2(u-1) !\left(\frac{p^{2}+\alpha^{2}}{2}\right)^{\frac{q+1}{2}}} F_{1}\left(\frac{q+1}{2} ; u ; \frac{\beta^{2}}{2} \frac{\alpha^{2}}{p^{2}+\alpha^{2}}\right), \quad q>-1,
\end{aligned}
$$

we can solve $I$ by evaluating $G_{u}$ recursively for $q>-1$ and restricted $u$ integer values as

$$
\begin{aligned}
& G_{u}=G_{u-1}+A_{u-1} F_{u} \\
& =G_{u-2}+A_{u-2} F_{u-2}+A_{u-1} F_{u-1} \\
& \vdots \\
& =G_{1}+\sum_{n=1}^{u-1} A_{n} F_{n+1},
\end{aligned}
$$

where $A_{n}$ and $F_{n}$ are given respectively, as

$$
\begin{gathered}
A_{n}=\frac{1}{2(n !)\left(\frac{p^{2}+\alpha^{2}}{2}\right)^{\frac{q+1}{2}}} \Gamma\left(\frac{q+1}{2}\right)\left(\frac{\beta^{2}}{2}\right)^{n} e^{-\frac{\beta^{2}}{2}}, \\
F_{n}={ }_{1} F_{1}\left(\frac{q+1}{2} ; n ; \frac{\beta^{2}}{2} \frac{\alpha^{2}}{p^{2}+\alpha^{2}}\right),
\end{gathered}
$$

where, ${ }_{1} F_{1}(., . ;$.$) as defined in (3.5)$. Hence, the solution of $(5.4)$ is given as $[86,87]$

$$
I_{A}=\frac{1}{2^{m-1}}\left[G_{1}+\frac{\eta}{2} \sum_{n=1}^{u-1} \frac{1}{n !}\left(\frac{\lambda}{2}\right)^{n}{ }_{1} F_{1}\left(m ; n+1 ; \frac{\lambda \bar{\gamma}}{2(m+\bar{\gamma})}\right)\right]
$$


where $\eta=\Gamma(m)\left(\frac{2 \bar{\gamma}}{m+\bar{\gamma}}\right)^{m} e^{-\frac{\lambda}{2}}$ and $G_{1}$ can be obtained by evaluating the following integral containing the first order of Marcum $Q$-function $Q(.,$.$) for integer m$ values as

$$
G_{1}=\int_{0}^{\infty} Q(x, \sqrt{\lambda}) x^{2 m-1} e^{-\frac{m x^{2}}{2 \bar{\gamma}}} \mathrm{d} x
$$

Using [[81], Eq. (25)], we evaluate $G_{1}$ for integer $m$ values as $[86,87]$

$$
\begin{aligned}
G_{1}=\frac{2^{m-1}(m-1) !}{\left(\frac{m}{\bar{\gamma}}\right)^{2 m}}\left(\frac{\bar{\gamma}}{m+\bar{\gamma}}\right) e^{-\frac{\lambda}{2} \frac{m}{m+\bar{\gamma}}} & \\
\times & {\left[\left(\frac{m+\bar{\gamma}}{\bar{\gamma}}\right)\left(\frac{m}{m+\bar{\gamma}}\right)^{m-1} L_{m-1}\left(-\frac{\lambda \bar{\gamma}}{2(m+\bar{\gamma})}\right)\right.} \\
& \left.+\sum_{n=0}^{m-2}\left(\frac{m}{m+\bar{\gamma}}\right)^{n} L_{n}\left(-\frac{\lambda \bar{\gamma}}{2(m+\bar{\gamma})}\right)\right] .
\end{aligned}
$$

Here $L_{n}($.$) denotes Laguerre polynomial of n$-degree [77].

\section{Evaluation of Integral $I_{B}$}

The integral $I_{B}$ in (5.3) is

$$
I_{B}=\underbrace{\int_{0}^{\infty} Q_{u}(\sqrt{2 \gamma}, \sqrt{\lambda}) \gamma^{m-1} \exp \left(-\frac{m \gamma}{\bar{\gamma}}\right) Q_{m}(\sqrt{2 a \rho \gamma}, \sqrt{2 a \gamma}) \mathrm{d} \gamma}_{I_{B}}
$$

Using the alternative representations for $Q_{u}(\sqrt{2 \gamma}, \sqrt{\lambda})$ given in (3.19) and the 
alternative representation given in [[19], Eq. (4.74)] for restricted $m$ integer values as

$$
Q_{m}\left(\alpha_{1}, \beta_{1}\right)=\sum_{i=0}^{\infty} \exp \left(-\frac{\alpha_{1}^{2}}{2}\right) \frac{\left(\frac{\alpha_{1}^{2}}{2}\right)^{i}}{i !} \sum_{k=0}^{i+m-1} \exp \left(-\frac{\beta_{1}^{2}}{2}\right) \frac{\left(\frac{\beta_{1}^{2}}{2}\right)^{k}}{k !}
$$

therefore, $Q_{m}(\sqrt{2 a \rho \gamma}, \sqrt{2 a \gamma})$ could be written as

$$
Q_{m}(\sqrt{2 a \rho \gamma}, \sqrt{2 a \gamma})=\sum_{i=0}^{\infty} \sum_{k=0}^{i+m-1} \frac{a^{i+k} \rho^{i}}{i ! k !} e^{-a \gamma(\rho+1)} \gamma^{i+k}
$$

Then by substituting into (5.12) with some simplification and rearranging, we can show

$$
\begin{array}{r}
I_{B}=\sum_{n=0}^{\infty} \sum_{i=0}^{\infty} \sum_{k=0}^{i+m-1} \frac{\Gamma\left(u+n, \frac{\lambda}{2}\right)}{\Gamma(u+n) n !} \frac{a^{i+k} \rho^{i}}{i ! k !} \\
\times \underbrace{\int_{0}^{\infty} \gamma^{i+k+m+n-1} \exp \left[-\gamma\left(1+a(\rho+1)+\frac{m}{\bar{\gamma}}\right)\right] \mathrm{d} \gamma}_{I} .
\end{array}
$$

Now, the next task is solving the integral $I$ in (5.15). For this we use [[77], Eq. (3.351/3)] and satisfying the condition therein

$$
\int_{0}^{\infty} x^{p_{1}} e^{-\mu_{1} x} \mathrm{~d} x=p_{1} ! \mu_{1}^{-p_{1}-1} \quad\left[\operatorname{Re} \mu_{1}>0\right]
$$

Hence (5.15) becomes $[86,87]$

$$
I_{B}=\sum_{n=0}^{\infty} \sum_{i=0}^{\infty} \sum_{k=0}^{i+m-1} \frac{\Gamma\left(u+n, \frac{\lambda}{2}\right)}{\Gamma(u+n)} \frac{a^{i+k} \rho^{i}}{n ! i ! k !} \frac{(i+k+m+n-1) !}{\left(1+a(\rho+1)+\frac{m}{\bar{\gamma}}\right)^{n+i+k+m}} .
$$

Substituting (5.9) and (5.17) into (5.3), hence, the average detection probability for 
dual correlated identical Nakagami- $m$ fading branches with SC diversity restricted to integer $u$ and $m$ values is $[86,87]$

$$
\begin{aligned}
\bar{P}_{D, S C, 2}=\frac{2}{\Gamma(m)}\left(\frac{m}{\bar{\gamma}}\right)^{m} & {\left[\frac{1}{2^{m-1}}\left\{G_{1}+\frac{\eta}{2} \sum_{n=1}^{u-1} \frac{1}{n !}\left(\frac{\lambda}{2}\right)^{n}{ }_{1} F_{1}\left(m ; n+1 ; \frac{\lambda \bar{\gamma}}{2(m+\bar{\gamma})}\right)\right\}\right.} \\
& \left.-\sum_{n=0}^{\infty} \sum_{i=0}^{\infty} \sum_{k=0}^{i+m-1} \frac{\Gamma\left(u+n, \frac{\lambda}{2}\right)}{\Gamma(u+n) n !} \frac{a^{i+k} \rho^{i}(i+k+m+n-1) !}{\left(1+a(\rho+1)+\frac{m}{\bar{\gamma}}\right)^{i+k+m+n} i ! k !}\right] .
\end{aligned}
$$

For $m=1$, (5.18) reduces to dual correlated Rayleigh fading branches.

It's worthwhile to mention that for i.i.d. fading diversity branches, (5.18) reduces to [[24], Eq. (7), [23], Eq. (20)) multiplied by 2, which is equivalent to double (not exceeding unity) value of the average detection probability in flat fading, hence improved detection performance. We derive (5.18) to serve as a proof.

\subsection{2 $\quad \bar{P}_{D, S C, 2}$ Alternative Expression}

Despite the fact that the part $Q_{u}(\sqrt{2 \gamma}, \sqrt{\lambda})$ of the second integral $I_{B}$ in (5.3) is evaluated for non-restricted $u$ integer values, however still we consider (5.18) is restricted for integer values since the first integral $I_{A}$ in (5.3) is evaluated for integer $u$ and $m$ values. In this section we will derive a more general and simpler alternative expression for (5.18) which is not restricted to integer $u$ values.

Using the series representation of Marcum-Q function where $u$ is not restricted to integer values in (3.12) and (5.2) and, substituting into (2.12) and using the definition 
of the PDF in (3.13), yields

$$
\begin{aligned}
& \bar{P}_{D, S C, 2}=1- \underbrace{\int_{0}^{\infty} e^{-\frac{2 \gamma+\lambda}{2}} \sum_{n=u}^{\infty}\left(\frac{\sqrt{\lambda}}{\sqrt{2 \gamma}}\right)^{n} I_{n}(\sqrt{2 \lambda \gamma}) \frac{2}{\Gamma(m)}\left(\frac{m}{\bar{\gamma}}\right)^{m} \gamma^{m-1} \exp \left(\frac{-m \gamma}{\bar{\gamma}}\right) \mathrm{d} \gamma}_{I_{A}} \\
&+\underbrace{\int_{0}^{\infty} e^{-\frac{2 \gamma+\lambda}{2}} \sum_{n=u}^{\infty}\left(\frac{\sqrt{\lambda}}{\sqrt{2 \gamma}}\right)^{n} I_{n}(\sqrt{2 \lambda \gamma}) \frac{2}{\Gamma(m)}\left(\frac{m}{\bar{\gamma}}\right)^{m} \gamma^{m-1} \exp \left(\frac{-m \gamma}{\bar{\gamma}}\right)}_{I_{B}} \\
& \times \underbrace{Q_{m}(\sqrt{2 a \rho \gamma}, \sqrt{2 a \gamma})}_{I_{B}} \mathrm{~d} \gamma, \quad \gamma \geq 0 .
\end{aligned}
$$

\section{Evaluation of Integral $I_{A}$}

Simplifying and rearranging the integral $I_{A}$ in (5.19), yields

$$
I_{A}=\frac{2}{\Gamma(m)}\left(\frac{m}{\bar{\gamma}}\right)^{m} e^{-\frac{\lambda}{2}} \sum_{n=u}^{\infty}\left(\frac{\lambda}{2}\right)^{\frac{n}{2}} \underbrace{\int_{0}^{\infty} \gamma^{m-\frac{n}{2}-1} e^{-\gamma\left(1+\frac{m}{\bar{\gamma}}\right)} I_{n}(\sqrt{2 \lambda \gamma}) \mathrm{d} \gamma}_{I_{a}}
$$

Using (3.16) and (3.17) and satisfying the condition in (3.16), hence, the solution of the integral $I_{a}$ in $(5.20)$ is

$$
I_{a}=\frac{\Gamma(m)}{\Gamma(n+1)}\left(\frac{\lambda}{2}\right)^{\frac{n}{2}} d_{1}^{-m} F_{1}\left(m ; 1+n ; \frac{\lambda}{2 d}\right)
$$

Substituting (5.21) in (5.20) with some simplification, the solution of $I_{A}$ is [87]

$$
I_{A}=\frac{2}{d^{m}}\left(\frac{m}{\bar{\gamma}}\right)^{m} e^{-\frac{\lambda}{2}} \sum_{n=u}^{\infty}\left(\frac{\lambda}{2}\right)^{n} \frac{1}{\Gamma(n+1)}{ }_{1} F_{1}\left(m ; 1+n ; \frac{\lambda}{2 d}\right)
$$


where $d=\left(\frac{\bar{\gamma}+m}{\bar{\gamma}}\right)$.

\section{Evaluation of Integral $I_{B}$}

The integral $I_{B}$ in (5.19) is

$$
\begin{aligned}
& I_{B}=\underbrace{\int_{0}^{\infty} e^{-\frac{2 \gamma+\lambda}{2}} \sum_{n=u}^{\infty}\left(\frac{\sqrt{\lambda}}{\sqrt{2 \gamma}}\right)^{n} I_{n}(\sqrt{2 \lambda \gamma}) \frac{2}{\Gamma(m)}\left(\frac{m}{\bar{\gamma}}\right)^{m} \gamma^{m-1} \exp \left(\frac{-m \gamma}{\bar{\gamma}}\right)}_{I_{B}} \\
& \times \underbrace{Q_{m}(\sqrt{2 a \rho \gamma}, \sqrt{2 a \gamma}) \mathrm{d} \gamma}_{I_{B}} .
\end{aligned}
$$

Using the alternative representation for $Q_{m}(\sqrt{2 a \rho \gamma}, \sqrt{2 a \gamma})$ given in (5.14) then simplifying and rearranging, $I_{B}$ in (5.19) becomes

$$
\begin{aligned}
I_{B} & =\frac{2}{\Gamma(m)}\left(\frac{m}{\bar{\gamma}}\right)^{m} e^{-\frac{\lambda}{2}} \sum_{n=u}^{\infty} \sum_{i=0}^{\infty} \sum_{k=0}^{i+m-1}\left(\frac{\lambda}{2}\right)^{\frac{n}{2}} \frac{a^{i+k} \rho^{i}}{i ! k !} \\
& \times \underbrace{\int_{0}^{\infty} \gamma^{m-\frac{n}{2}+i+k-1} e^{-\gamma(d+a(\rho+1))} I_{n}(\sqrt{2 \lambda \gamma}) \mathrm{d} \gamma}_{I_{b}} .
\end{aligned}
$$


Following same procedures as in (5.22) and satisfying the condition therein to solve the integral $I_{b}$ in (5.24), yields [87]

$$
\begin{aligned}
I_{B}=\frac{2}{\Gamma(m)}\left(\frac{m}{\bar{\gamma}}\right)^{m} e^{-\frac{\lambda}{2}} \sum_{n=u}^{\infty} \sum_{i=0}^{\infty} \sum_{k=0}^{i+m-1} & \left(\frac{\lambda}{2}\right)^{n} \frac{a^{i+k} \rho^{i}}{i ! k !} \frac{\Gamma(m+i+k)}{\Gamma(n+1) q^{m+i+k}} \\
& \times{ }_{1} F_{1}\left(m+i+k ; 1+n ; \frac{\lambda}{2 q}\right)
\end{aligned}
$$

where $a=\frac{m}{\bar{\gamma}(1-\rho)}, d=\left(\frac{\bar{\gamma}+m}{\bar{\gamma}}\right)$ and $q=d+a(\rho+1)$.

Substituting (5.22) and (5.25) into (5.19), hence, for $u$ is not-restricted while $m$ is restricted to integer values, the average detection probability is [87]

$$
\begin{array}{r}
\bar{P}_{D, S C, 2}=1-2\left(\frac{m}{\bar{\gamma}}\right)^{m} e^{-\frac{\lambda}{2}}\left[\frac{1}{d^{m}} \sum_{n=u}^{\infty}\left(\frac{\lambda}{2}\right)^{n} \frac{1}{n !}{ }_{1} F_{1}\left(m ; 1+n ; \frac{\lambda}{2 d}\right)\right. \\
\left.-\frac{1}{\Gamma(m)} \sum_{n=u}^{\infty} \sum_{i=0}^{\infty} \sum_{k=0}^{i+m-1}\left(\frac{\lambda}{2}\right)^{n} \frac{\Gamma(m+i+k) a^{i+k} \rho^{i}}{q^{m+i+k} n ! i ! k !}{ }_{1} F_{1}\left(m+i+k ; 1+n ; \frac{\lambda}{2 q}\right)\right],
\end{array}
$$

For $m=1$, (5.26) reduces to dual correlated Rayleigh fading branches and for i.i.d multipath fading reduces to [[34], Eq. (59)] with dual diversity branches.

Fortunately, the error results from truncating the infinite series in (5.26) is upper bounded by the Confluent Hypergeometric functions defined in (3.5). Since this function is monotonically decreasing [80] in $i, k$ and $n$ for given values of $m, \lambda$ and $\bar{\gamma}$, hence, the number of $N$-terms required for five digit accuracy could be calculated using this constraint as shown in Table 5.1. 


\subsection{Triple Exponentially Correlated SC}

In this section, we extend the derivations of the average detection probability to triple correlated diversity branches. For triple exponentially correlated Nakagami- $m$ branches with SC, the PDF of the output SNR can be obtained from the corresponding trivariate Nakagami-m's PDF of the fading envelope given by [[88], Eq. (8)]. Therefore, by changing variable and assuming identical branches $\left(\bar{\gamma}=\overline{\gamma_{1}}=\overline{\gamma_{2}}=\overline{\gamma_{3}}\right.$, and the same fading parameter $m$ ) this output SNR's PDF is,

$$
p_{\gamma S C, 3}(\gamma)=\frac{\left|\Sigma^{-1}\right|^{m}}{\Gamma(m)} \sum_{i=0}^{\infty} \sum_{j=0}^{\infty} \frac{\left|p_{1,2}\right|^{2 i}\left|p_{2,3}\right|^{2 j}}{p_{1,1}^{i+m} p_{2,2}^{i+j+m} p_{3,3}^{j+m}} \frac{\left[\Theta_{1}+\Theta_{2}+\Theta_{3}\right]}{\Gamma(m+i) \Gamma(m+j) i ! j !},
$$

where $\boldsymbol{\Sigma}^{-1}$ is the inverse of the correlation matrix, $p_{i_{1}, j_{1}}\left(i_{1}, j_{1}=1,2,3\right)$ being its entries and $\Theta_{1}, \Theta_{2}$ and $\Theta_{3}$ are respectively

$$
\begin{aligned}
& \Theta_{1}=\left(\frac{p_{1,1} m}{\bar{\gamma}}\right)^{i+m} \gamma^{i+m-1} e^{-\frac{p_{1,1} m}{\bar{\gamma}} \gamma} \gamma\left(i+j+m, \frac{p_{2,2} m}{\bar{\gamma}} \gamma\right) \gamma\left(j+m, \frac{p_{3,3} m}{\bar{\gamma}} \gamma\right), \\
& \Theta_{2}=\left(\frac{p_{2,2} m}{\bar{\gamma}}\right)^{i+j+m} \gamma^{i+j+m-1} e^{-\frac{p_{2,2} m}{\bar{\gamma}} \gamma} \gamma\left(i+m, \frac{p_{1,1} m}{\bar{\gamma}} \gamma\right) \gamma\left(j+m, \frac{p_{3,3} m}{\bar{\gamma}} \gamma\right), \\
& \Theta_{3}=\left(\frac{p_{3,3} m}{\bar{\gamma}}\right)^{j+m} \gamma^{j+m-1} e^{-\frac{p_{3,3} m}{\bar{\gamma}}} \gamma\left(i+m, \frac{p_{1,1} m}{\bar{\gamma}} \gamma\right) \gamma\left(i+j+m, \frac{p_{2,2} m}{\bar{\gamma}} \gamma\right) .
\end{aligned}
$$

Here $\gamma(a, x)$ denotes the lower incomplete gamma function defined as $\int_{0}^{x} e^{-t} t^{a-1} \mathrm{~d} t$ in [[77], Eq. (8.350/1)]. 
In exponentially correlation model, the diversity antennas are equispaced, and the correlation matrix can be written as $\Sigma_{i_{1}, j_{1}} \equiv \rho^{\left|i_{1}-j_{1}\right|}[66]$. Hence, it easy to show that the inverse correlation matrix $\boldsymbol{\Sigma}^{-1}$ is tridiagonal and can be written as

$$
\Sigma^{-1}=\frac{1}{\rho^{2}-1}\left[\begin{array}{rrr}
-1 & \rho & 0 \\
\rho & -\left(\rho^{2}+1\right) & \rho \\
0 & \rho & -1
\end{array}\right]
$$

where, $\rho$ denotes the correlation coefficient.

The assumption we made above that identical average SNRs in all three branches is reasonable if the diversity channels are closely spaced and the gain of each channel is such that all the noise powers are equals [19].

\subsubsection{Average Detection Probability, $\bar{P}_{D, S C, 3}$}

Using (5.13) and substituting (5.27) into (2.12), the average detection probability is,

$$
\begin{array}{r}
\bar{P}_{D, S C, 3}=\frac{\left|\boldsymbol{\Sigma}^{-1}\right|^{m}}{\Gamma(m)} e^{-\frac{\lambda}{2}} \sum_{n=0}^{\infty} \sum_{k=0}^{n+u-1} \sum_{i=0}^{\infty} \sum_{j=0}^{\infty} \frac{\left|p_{1,2}\right|^{2 i}\left|p_{2,3}\right|^{2 j}\left(\frac{\lambda}{2}\right)^{k}}{p_{1,1}^{i+m} p_{2,2}^{i+j+m} p_{3,3}^{j+m} \Gamma(m+i) \Gamma(m+j) i ! j ! k ! n !} \\
\times \underbrace{\int_{0}^{\infty} \gamma^{n} e^{-\gamma}\left[\Theta_{1}+\Theta_{2}+\Theta_{3}\right] \mathrm{d} \gamma}_{I_{A}}
\end{array}
$$


Substituting (5.28), (5.29) and (5.30) into (5.32) and simplifying, the integral part $I_{A}$ in (5.32) becomes

$$
I_{A}=\left(\frac{p_{1,1} m}{\bar{\gamma}}\right)^{i+m} I_{a 1}+\left(\frac{p_{2,2} m}{\bar{\gamma}}\right)^{i+j+m} I_{a 2}+\left(\frac{p_{3,3} m}{\bar{\gamma}}\right)^{j+m} I_{a 3}
$$

where,

$$
\begin{aligned}
& I_{a 1}=\int_{0}^{\infty} \gamma^{i+m+n-1} e^{-\gamma\left(\frac{p_{11} m}{\bar{\gamma}}+1\right)} \gamma\left(i+j+m, \frac{p_{2,2} m}{\bar{\gamma}} \gamma\right) \gamma\left(j+m, \frac{p_{3,3} m}{\bar{\gamma}} \gamma\right) \mathrm{d} \gamma \\
& I_{a 2}=\int_{0}^{\infty} \gamma^{i+j+m+n-1} e^{-\gamma\left(\frac{p_{2,2} m}{\bar{\gamma}}+1\right)} \gamma\left(i+m, \frac{p_{1,1} m}{\bar{\gamma}} \gamma\right) \gamma\left(j+m, \frac{p_{3,3} m}{\bar{\gamma}} \gamma\right) \mathrm{d} \gamma \\
& I_{a 3}=\int_{0}^{\infty} \gamma^{j+m+n-1} e^{-\gamma\left(\frac{p_{3,3} m}{\bar{\gamma}}+1\right)} \gamma\left(i+m, \frac{p_{1,1} m}{\bar{\gamma}} \gamma\right) \gamma\left(i+j+m, \frac{p_{2,2} m}{\bar{\gamma}} \gamma\right) \mathrm{d} \gamma .
\end{aligned}
$$

Each integral in (5.33) could be written as

$$
I=\int_{0}^{\infty} x^{a} e^{-b x} \gamma\left(d_{1}, c_{1} x\right) \gamma\left(d_{2}, c_{2} x\right) \mathrm{d} x
$$

Using [[89], Eq. (10)], and after lengthy simplification and rearrangement, the solution of $(5.33)$ is

$$
I_{A}=\left(\frac{\bar{\gamma}}{m}\right)^{n} \frac{p_{1,1}^{i+m} p_{2,2}^{i+j+m} p_{3,3}^{j+m} \Gamma(2 i+2 j+3 m+n)}{\left(p_{11}+p_{2,2}+p_{3,3}+\frac{\bar{\gamma}}{m}\right)^{(2 i+2 j+3 m+n)}}\left(\Xi_{1}+\Xi_{2}+\Xi_{3}\right),
$$


where $\Xi_{1}, \Xi_{2}$ and $\Xi_{3}$ are defined below in (5.39), (5.40) and (5.41) respectively.

$\Xi_{1}=\frac{F_{2}\left(2 i+2 j+3 m+n ; 1,1 ; i+j+m+1, j+m+1 ; \frac{p_{2,2}}{p_{11}+p_{2,2}+p_{3,3}+\frac{\gamma}{m}}, \frac{p_{3,3}}{p_{11}+p_{2,2}+p_{3,3}+\frac{\gamma}{m}}\right)}{(i+j+m)(j+m)}$,

$\Xi_{2}=\frac{F_{2}\left(2 i+2 j+3 m+n ; 1,1 ; i+m+1, j+m+1 ; \frac{p_{1,1}}{p_{11}+p_{2,2}+p_{3,3}+\frac{\bar{\gamma}}{m}}, \frac{p_{3,3}}{p_{11}+p_{2,2}+p_{3,3}+\frac{\bar{\gamma}}{m}}\right)}{(i+m)(j+m)}$,

$\Xi_{3}=\frac{F_{2}\left(2 i+2 j+3 m+n ; 1,1 ; i+m+1, i+j+m+1 ; \frac{p_{1,1}}{p_{11}+p_{2,2}+p_{3,3}+\frac{\gamma}{m}}, \frac{p_{2,2}}{p_{11}+p_{2,2}+p_{3,3}+\frac{\gamma}{m}}\right)}{(i+m)(i+j+m)}$,

where $F_{2}\left(\alpha_{3} ; \beta_{3}, \beta_{3}^{\prime} ; \gamma_{3}, \gamma_{3}^{\prime} ; x, y\right)$ denotes the Hypergeometric function of two variables defined in $([77],(9.180 .2))$.

Substituting (5.38) in (5.32) hence, the average detection probability for triple Nakagami- $m$ correlated branches with SC diversity for $u$ is restricted while $m$ is not restricted to integer values could be obtained as $[86,87]$

$$
\begin{aligned}
\bar{P}_{D, S C, 3}=\frac{\left|\Sigma^{-1}\right|^{m}}{\Gamma(m)} e^{-\frac{\lambda}{2}} \sum_{n=0}^{\infty} \sum_{k=0}^{n+u-1} \sum_{i=0}^{\infty} \sum_{j=0}^{\infty} \\
\quad\left[\left(\frac{\lambda}{2}\right)^{k}\left(\frac{\bar{\gamma}}{m}\right)^{n} \frac{\left|p_{1,2}\right|^{2 i}\left|p_{2,3}\right|^{2 j}}{p_{1,1}^{i+m} p_{2,2}^{i+j+m} p_{3,3}^{j+m} \Gamma(m+i) \Gamma(m+j)}\right. \\
\left.\times \frac{p_{1,1}^{i+m} p_{2,2}^{i+j+m} p_{3,3}^{j+m} \Gamma(2 i+2 j+3 m+n)}{\left(p_{11}+p_{2,2}+p_{3,3}+\frac{\bar{\gamma}}{m}\right)^{(2 i+2 j+3 m+n)} i ! j ! k ! n !}\left(\Xi_{1}+\Xi_{2}+\Xi_{3}\right)\right] .
\end{aligned}
$$

Here $\Xi_{1}, \Xi_{2}$ and $\Xi_{3}$ are defined in (5.39), (5.40) and (5.41) respectively. For $m=1$, (5.42) reduces to triple correlated Rayleigh fading branches. 


\subsubsection{Alternative $\bar{P}_{D, S C, 3}$ Expression}

In this section, we derive a general and simpler alternative expression to (5.42) where both $u$ and $m$ are not restricted to integer values. Using (3.19) and substituting (5.27) into (2.12) with rearranging, yields,

$$
\begin{array}{r}
\bar{P}_{D, S C, 3}=\frac{\left|\Sigma^{-1}\right|^{m}}{\Gamma(m)} \sum_{n=0}^{\infty} \sum_{i=0}^{\infty} \sum_{j=0}^{\infty} \frac{\Gamma\left(u+n, \frac{\lambda}{2}\right)}{\Gamma(u+n)} \frac{\left|p_{1,2}\right|^{2 i}\left|p_{2,3}\right|^{2 j}}{p_{1,1}^{i+m} p_{2,2}^{i+j+m} p_{3,3}^{j+m} \Gamma(m+i) \Gamma(m+j) i ! j ! n !} \\
\times \underbrace{\int_{0}^{\infty} \gamma^{n} e^{-\gamma}\left[\Theta_{1}+\Theta_{2}+\Theta_{3}\right] \mathrm{d} \gamma}_{I_{A}}
\end{array}
$$

Following same procedures in (5.33)-(5.38), then substituting (5.38) into (5.43), hence, the average detection probability for triple Nakagami- $m$ correlated branches with SC diversity for not restricted $u$ or $m$ integer values can be obtained as $[86,87]$

$$
\begin{aligned}
& \bar{P}_{D, S C, 3}=\frac{\left|\Sigma^{-1}\right|^{m}}{\Gamma(m)} \sum_{n=0}^{\infty} \sum_{i=0}^{\infty} \sum_{j=0}^{\infty}\left(\frac{\bar{\gamma}}{m}\right)^{n} \frac{\Gamma\left(u+n, \frac{\lambda}{2}\right)}{\Gamma(u+n)} \frac{\left|p_{1,2}\right|^{2 i}\left|p_{2,3}\right|^{2 j} \Gamma(2 i+2 j+3 m+n)}{\Gamma(m+i) \Gamma(m+j) i ! j ! n !} \\
& \times \frac{\left(\Xi_{1}+\Xi_{2}+\Xi_{3}\right)}{\left(p_{11}+p_{2,2}+p_{3,3}+\frac{\bar{\gamma}}{m}\right)^{2 i+2 j+3 m+n}}
\end{aligned}
$$

where, $\Xi_{1}, \Xi_{2}$ and $\Xi_{3}$ are defined in (5.39), (5.40) and (5.41) respectively. Similarly, for $m=1,(5.44)$ reduces to triple correlated Rayleigh fading branches. It's worthwhile to mention that the Hypergeometric function of two variables $F_{2}\left(\alpha_{3} ; \beta_{3}, \beta_{3}^{\prime} ; \gamma_{3}, \gamma_{3}^{\prime} ; x, y\right)$ appears in (5.42) and (5.44) converges for $|x|+|y|<1[77]$, which is the case in our above derived equations. 
Table 5.1: Terms required for five digits accuracy

\begin{tabular}{|l|l|l|l|l|l|}
\hline \multicolumn{2}{|c|}{$\bar{P}_{D, S C, 2}:$} & $\tilde{E}_{N}$ & \multicolumn{3}{|c|}{$u=2, P_{F}=0.01, \bar{\gamma}=20 \mathrm{~dB}$} \\
\hline$\rho$ & $m=1$ & $m=2$ & $m=3$ & $m=4$ \\
& $N_{n}, N_{i}$ & $N_{n}, N_{i}$ & $N_{n}, N_{i}$ & $N_{n}, N_{i}$ \\
\hline 0 & 15,1 & 15,1 & 15,1 & 15,1 \\
\hline 0.2 & 15,3 & 15,3 & 15,2 & 15,1 \\
\hline 0.4 & 15,3 & 15,2 & 15,1 & 15,1 \\
\hline 0.6 & 15,3 & 15,5 & 15,4 & 15,4 \\
\hline 0.8 & 15,4 & 15,5 & 15,6 & 15,7 \\
\hline
\end{tabular}

\subsection{Performance Analysis}

In this section, we verify the derived closed-form expressions for different cases of interest for spectrum sensing model with SC technique. The impact of multipath fading and correlation on average detection probability (as a performance factor) is investigated.

We follow same procedures as we did in Sec. 3.4 to calculate the corresponding threshold for the assumed detection parameters to produce corresponding SC' CROC graphs. Table 5.1 is constructed to demonstrate the number of terms required for five digit accuracy for the infinite series truncations.

In Figure. 5.2, numerical results obtained for analytic and simulation are almost in a perfect match. This validates the derived expressions.

CROC curves for dual correlated Nakagami- $m$ fading branches with SC diversity are shown in Figure 5.3. The performance variation has to be observed with respect fading parameter $m$ and correlation $\rho$ values. One can clearly observe the deterioration in the performance as $\rho$ increases. 


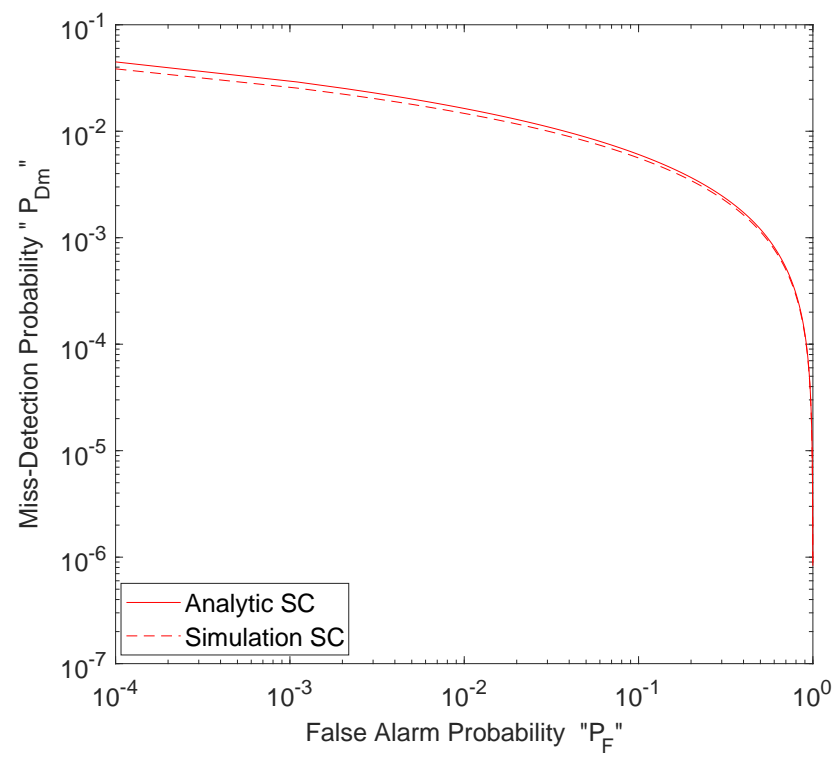

Figure 5.2: Analytic (solid) versus simulation (dashed) results for SC with $L=2, m=$ $1, \bar{\gamma}=20 \mathrm{~dB}$ and $\rho=0.8$.

For instance, let us consider the case $m=1$ and constant $P_{F}=0.01$ as in Figure 5.3a. The corresponding $P_{D m}$ for $\rho=0.8$ is almost four times its value for $\rho=$ 0, no correlation. Similar result could be observed in Figure 5.3b, however, the increment ratio is now much more larger. However, as $m$ increases (low fading environment), correlation effect is compensated for, resulting in higher detection probability (equivalently, low miss detection probability). Thus, the rate of correlation compensation due to good channel is higher than the correlation impact on detection probability. Therefore, we can conclude, for deep fading environment, correlation must be considered for accurate cognitive detection, while for low fading environment, its effect may be ignored. 


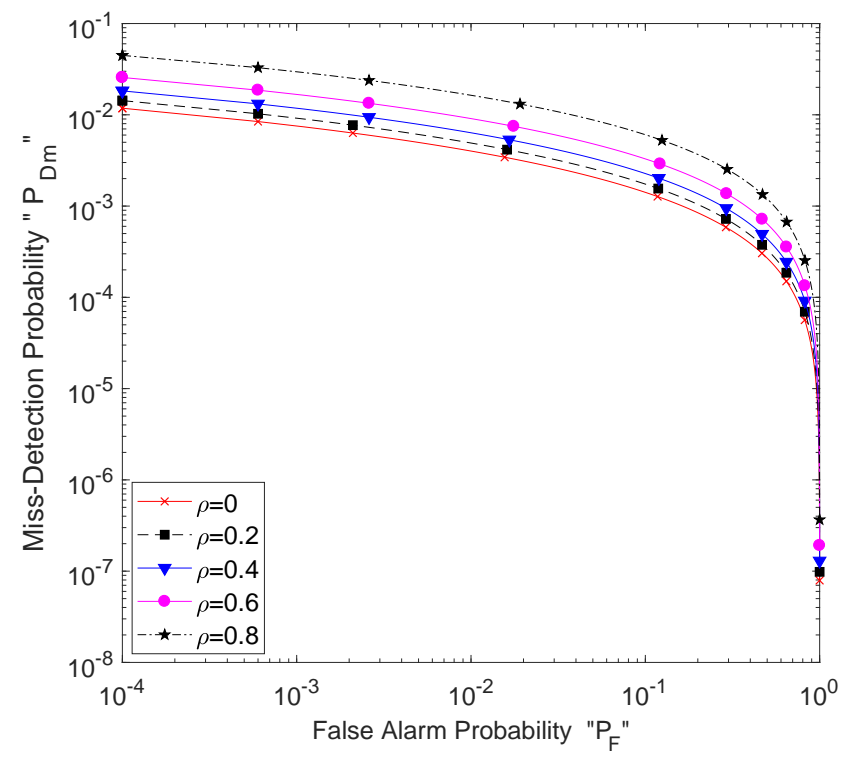

(a) $m=1$ (Rayleigh: deep fading)

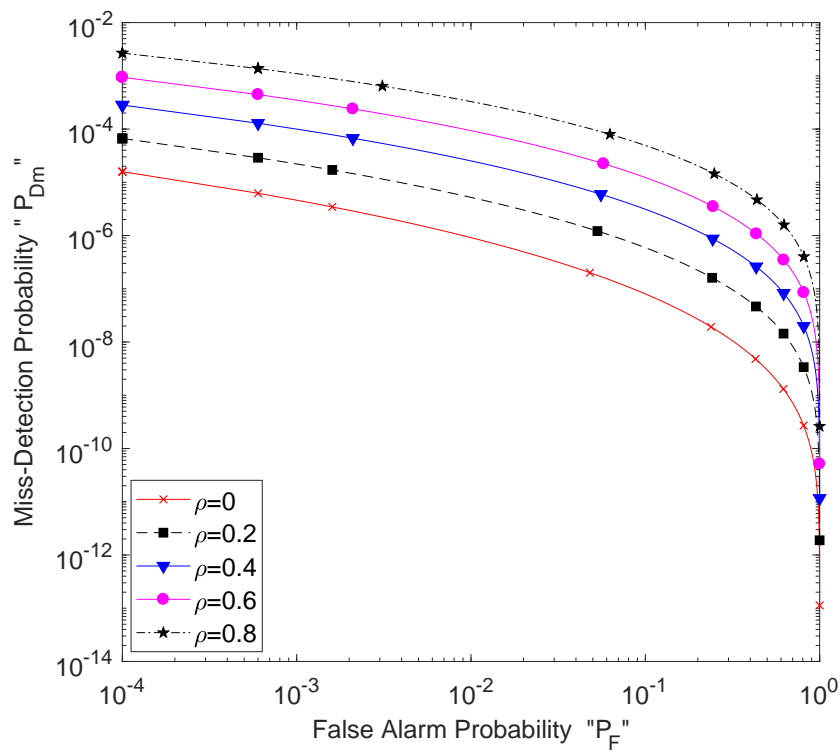

(b) $m=4$ (Low fading)

Figure 5.3: SC dual correlated Nakagami- $m$ branches with $\bar{\gamma}=20 \mathrm{~dB}$ for different $\rho$ values. 


\section{Chapter 6}

\section{Dual Correlated Nakagami- $m$ Branches with SSC Diversity}

In this chapter, we consider a detection scenario when SSC diversity technique is employed for dual diversity branches. We derive the average detection probability for dual Nakagami- $m$ correlated fading branches with SSC diversity. We do performance analysis using CROC graphs to evaluate the derived expressions by studying the correlation impact on the detection probability.

\subsection{SSC Diversity}

For a predetermined threshold, SSC's receiver selects a particular diversity branch until it's SNR drops below this predetermined value, then the receiver switches to 


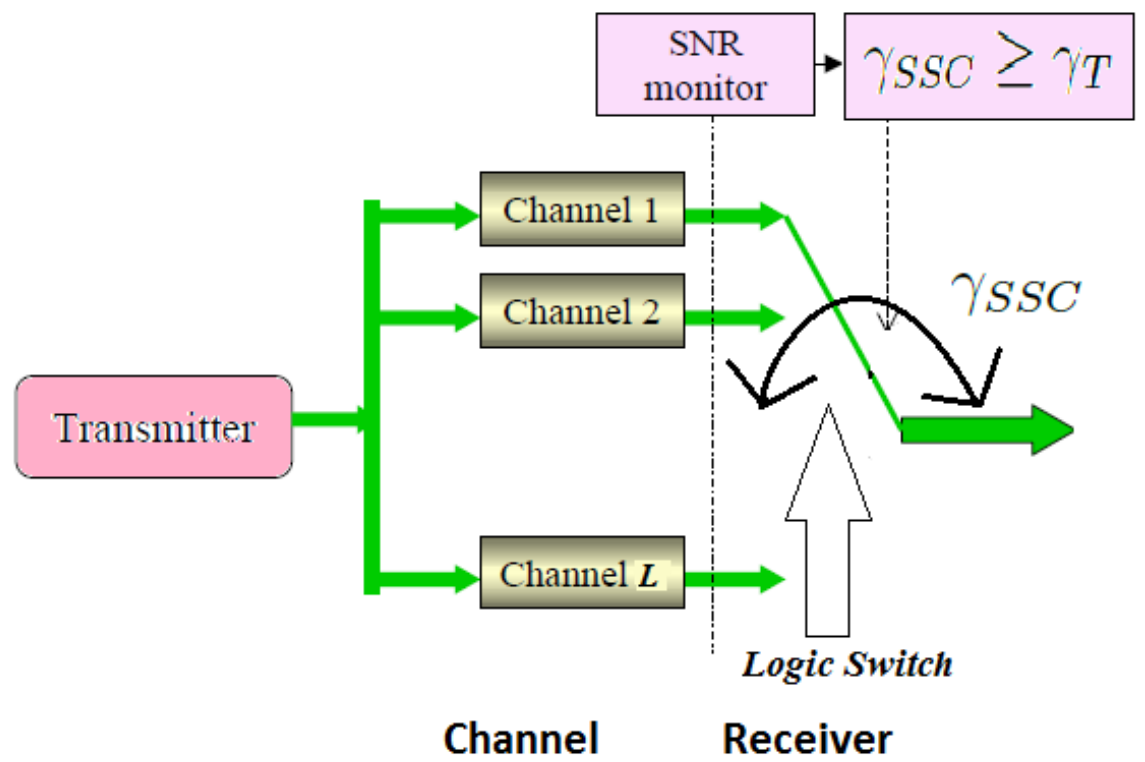

Figure 6.1: Switched and Stay Combining Technique (SSC).

another branch as shown in Figure $6.1^{1}$.

In fact, SSC receiver monitors the level of the incoming signal using logic switch. When the signal level drops below a predefined threshold, a switch changes the path to another branch (antenna) and so on. Therefore, SSC has less complexity than that for its counterpart SC since SSC does not continuously connect the diversity path with the best quality [19]. The drawback of selection SSC is that the switching process does not occur unless the fade occurs first. Moreover, it is possible that the signal at the other antenna will be at an even lower signal level. However, SSC is also less complicated than the two previous techniques, MRC and EGC since it just processes one branch with the highest SNR. Moreover, unlike MRC where full knowledge of CSI is required, SSC requires only the knowledge of the amplitude on the branches in

\footnotetext{
${ }^{1} \mathrm{SSC}$ combiner diagram is similar to that for $\mathrm{SC}$ as in Figure5.1 with only the exception mentioned above.
} 
order to select the highest one [19]. Finally, SSC can be used for both coherent and noncoherent modulation since no need for signal phase on each branch.

\subsection{Dual Arbitrarily Correlated Branches}

In this section, we will investigate arbitrarily dual correlated Nakagami- $m$ fading branches with SSC diversity. For dual and identical correlated Nakagami- $m$ fading channels with SSC combiner, the SNR's PDF is given by [[19], p.437, Eq. (9.334)] as

$$
p_{\gamma S S C}(\gamma)= \begin{cases}A(\gamma) & \gamma \leq \gamma_{T} \\ A(\gamma)+\left(\frac{m}{\bar{\gamma}}\right)^{m} \frac{\gamma^{m-1}}{\Gamma(m)} \exp \left(-\frac{m \gamma}{\bar{\gamma}}\right) & \gamma>\gamma_{T}\end{cases}
$$

where $\gamma_{T}$ denotes a predetermined switching threshold and $A(\gamma)$ is given by [[19], p.437, Eq. (9.335)] as

$$
A(\gamma)=\left(\frac{m}{\bar{\gamma}}\right)^{m} \frac{\gamma^{m-1}}{\Gamma(m)} \exp \left(-\frac{m \gamma}{\bar{\gamma}}\right)\left[1-Q_{m}\left(\sqrt{2 a \rho \gamma}, \sqrt{2 a \gamma_{T}}\right)\right]
$$

where $a=\frac{m}{\bar{\gamma}(1-\rho)}$ and $Q_{m}(.,$.$) , the Generalized Marcum Q-function. The average$ detection probability for dual correlated Nakagami- $m$ fading branches with SSC diversity $\left(\bar{P}_{D, S S C, 2}\right)$ is obtained by substituting (6.1) in (2.12) and then using the definition $\int_{a}^{\infty} f(x) \mathrm{d} x=\int_{0}^{\infty} f(x) \mathrm{d} x-\int_{0}^{a} f(x) \mathrm{d} x$ with some simplification and rearranging 
yields

$$
\begin{array}{r}
\bar{P}_{D, S S C, 2}=\frac{1}{\Gamma(m)}\left(\frac{m}{\bar{\gamma}}\right)^{m}[\underbrace{\left[2 \int_{0}^{\infty} Q_{u}(\sqrt{2 \gamma}, \sqrt{\lambda}) \gamma^{m-1} \exp \left(-\frac{m \gamma}{\bar{\gamma}}\right) \mathrm{d} \gamma\right.}_{I_{A}} \\
-\underbrace{\int_{0}^{\infty} Q_{u}(\sqrt{2 \gamma}, \sqrt{\lambda}) Q_{m}\left(\sqrt{2 a \rho \gamma}, \sqrt{2 a \gamma_{T}}\right) \gamma^{m-1} \exp \left(-\frac{m \gamma}{\bar{\gamma}}\right) \mathrm{d} \gamma}_{I_{B}} \\
-\underbrace{\left.\int_{0}^{\gamma_{T}} Q_{u}(\sqrt{2 \gamma}, \sqrt{\lambda}) \gamma^{m-1} \exp \left(-\frac{m \gamma}{\bar{\gamma}}\right) \mathrm{d} \gamma\right)}_{I_{C}} .
\end{array}
$$

Before deriving an expression for the detection probability $\bar{P}_{D, S S C, 2}$, it is worthy to investigate (6.3) for the following two special cases of threshold values.

\subsubsection{Case: $\gamma_{T}=0$}

If $\gamma_{T}=0$, hence $Q_{m}\left(\sqrt{2 a \rho \gamma}, \sqrt{2 a \gamma_{T}}\right)=1$ and the third term $I_{C}$ vanishes, consequently (6.3) reduces to a single branch detection given as

$$
\bar{P}_{D, S S C, 2}=\frac{1}{\Gamma(m)}\left(\frac{m}{\bar{\gamma}}\right)^{m} \int_{0}^{\infty} Q_{u}(\sqrt{2 \gamma}, \sqrt{\lambda}) \gamma^{m-1} \exp \left(-\frac{m \gamma}{\bar{\gamma}}\right) \mathrm{d} \gamma
$$

\subsubsection{Case: $\gamma_{T} \rightarrow \infty$}

If $\gamma_{T} \rightarrow \infty$, hence $Q_{m}\left(\sqrt{2 a \rho \gamma}, \sqrt{2 a \gamma_{T}}\right)=0$, consequently $I_{B}$ vanishes and only $I_{C}$ is subtracted from $I_{A}$. This results in a single branch detection too, as in (6.4). In the forthcoming writing, we will evaluate $\bar{P}_{D, S S C, 2}$ in (6.3). 


\subsubsection{Integral $I_{A}$ in (6.3)}

The integral $I_{A}$ in (6.3) is

$$
I_{A}=2 \int_{0}^{\infty} Q_{u}(\sqrt{2 \gamma}, \sqrt{\lambda}) \gamma^{m-1} \exp \left(-\frac{m \gamma}{\bar{\gamma}}\right) \mathrm{d} \gamma
$$

Using Marcum Q-function alternative representation (5.13) with some simplification and rearranging, (6.5) becomes,

$$
I_{A}=2 e^{-\frac{\lambda}{2}} \sum_{j=0}^{\infty} \sum_{k=0}^{j+u-1}\left(\frac{\lambda}{2}\right)^{k} \frac{1}{j ! k !} \int_{0}^{\infty} \gamma^{j+m-1} \exp \left\{-\gamma\left(1+\frac{m}{\bar{\gamma}}\right)\right\} \mathrm{d} \gamma
$$

Using (5.16) and satisfying the condition therein, the solution of (6.6) is [87]

$$
I_{A}=2 e^{-\frac{\lambda}{2}} \sum_{j=0}^{\infty} \sum_{k=0}^{j+u-1}\left(\frac{\lambda}{2}\right)^{k} \frac{(j+m-1) !}{j ! k !}\left(\frac{\bar{\gamma}}{\bar{\gamma}+m}\right)^{j+m}
$$

\subsubsection{Integral $I_{B}$ in (6.3)}

The integral in $I_{B}$ in (6.3) is

$$
I_{B}=\int_{0}^{\infty} Q_{u}(\sqrt{2 \gamma}, \sqrt{\lambda}) Q_{m}\left(\sqrt{2 a \rho \gamma}, \sqrt{2 a \gamma_{T}}\right) \gamma^{m-1} \exp \left(-\frac{m \gamma}{\bar{\gamma}}\right) \mathrm{d} \gamma
$$


We follow the same procedures as in (6.7) to evaluate both $Q_{u}(\sqrt{2 \gamma}, \sqrt{\lambda})$ and $Q_{m}\left(\sqrt{2 a \rho \gamma}, \sqrt{2 a \gamma_{T}}\right)$ with some simplification and rearranging. Then (6.8) becomes,

$$
\begin{aligned}
I_{B}= & e^{-\frac{\lambda}{2}} \sum_{n=0}^{\infty} \sum_{q=0}^{j+u-1} \sum_{i=0}^{\infty} \sum_{k=0}^{i+m-1}\left(\frac{\lambda}{2}\right)^{q} \frac{a^{i+k} \rho^{i}}{i ! k ! n ! q !} e^{-a \gamma_{T}} \gamma_{T}^{k} \\
& \times \int_{0}^{\infty} \gamma^{m+n+i-1} \exp \left\{-\gamma\left(a \rho+\frac{m}{\bar{\gamma}}+1\right)\right\} \mathrm{d} \gamma .
\end{aligned}
$$

Similarly, using (5.16) and satisfying the condition therein, the solution of $I_{B}$ is [87]

$$
I_{B}=e^{-\frac{\lambda}{2}} \sum_{n=0}^{\infty} \sum_{q=0}^{j+u-1} \sum_{i=0}^{\infty} \sum_{k=0}^{i+m-1}\left(\frac{\lambda}{2}\right)^{q} \frac{a^{i+k} \rho^{i}}{i ! k ! n ! q !} \frac{(m+n+i-1) !}{\left(a \rho+\frac{m}{\bar{\gamma}}+1\right)^{m+n+i}} e^{-a \gamma_{T}} \gamma_{T}^{k} .
$$

\subsubsection{Integral $I_{C}$ in (6.3)}

The Integral $I_{C}$ in $(6.3)$ is

$$
I_{C}=\int_{0}^{\gamma_{T}} Q_{u}(\sqrt{2 \gamma}, \sqrt{\lambda}) \gamma^{m-1} \exp \left(-\frac{m \gamma}{\bar{\gamma}}\right) \mathrm{d} \gamma
$$

Similarly, using (5.13), with some simplification and rearranging, (6.11) becomes,

$$
I_{C}=e^{-\frac{\lambda}{2}} \sum_{n=0}^{\infty} \sum_{q=0}^{n+u-1} \frac{1}{n ! q !}\left(\frac{\lambda}{2}\right)^{k} \int_{0}^{\gamma_{T}} \gamma^{m+n-1} \exp \left\{-\gamma\left(\frac{m}{\bar{\gamma}}+1\right)\right\} \mathrm{d} \gamma
$$

Using [[77], Eq. (3.351/1)] given as

$$
\begin{array}{r}
\int_{0}^{z} x^{n} e^{-\mu x} \mathrm{~d} x=\frac{n !}{\mu^{n+1}}-e^{-\mu z} \sum_{k=0}^{n} \frac{n !}{k !} \frac{z^{k}}{\mu^{n-k+1}}=\mu^{-n-1} \gamma(n+1, \mu z), \\
{[z>0, \operatorname{Re} \mu>0, n=0,1,2, \cdots],}
\end{array}
$$


and satisfying the conditions therein, hence, the solution of (6.12) is [87]

$$
I_{C}=e^{-\frac{\lambda}{2}} \sum_{n=0}^{\infty} \sum_{q=0}^{n+u-1} \frac{1}{n ! q !}\left(\frac{\lambda}{2}\right)^{q}\left(\frac{\bar{\gamma}}{\bar{\gamma}+m}\right)^{m+n} \gamma\left(m+n, \gamma_{T}\left(\frac{\bar{\gamma}+m}{\bar{\gamma}}\right)\right)
$$

Substituting (6.7), (6.10) and (6.14) in (6.3) and rearranging, hence, the average detection probability for dual correlated Nakagami- $m$ fading branches with SSC where $u$ and $m$ are restricted to integer values is [87]

$$
\begin{aligned}
\bar{P}_{D, S S C, 2} & =\frac{1}{\Gamma(m)}\left(\frac{m}{\bar{\gamma}}\right)^{m} e^{-\frac{\lambda}{2}}\left[2 \sum_{j=0}^{\infty} \sum_{k=0}^{j+u-1}\left(\frac{\lambda}{2}\right)^{k} \frac{(j+m-1) !}{j ! k !}\left(\frac{\bar{\gamma}}{\bar{\gamma}+m}\right)^{j+m}\right. \\
& -\sum_{n=0}^{\infty} \sum_{q=0}^{n+u-1} \sum_{i=0}^{\infty} \sum_{k=0}^{i+m-1}\left(\frac{\lambda}{2}\right)^{q} \frac{a^{i+k} \rho^{i}(m+n+i-1) !}{\left(a \rho+\frac{m}{\bar{\gamma}}+1\right)^{m+n+i} i ! k ! n ! q !} e^{-a \gamma_{T}} \gamma_{T}^{k} \\
& \left.-\sum_{n=0}^{\infty} \sum_{q=0}^{n+u-1}\left(\frac{\lambda}{2}\right)^{q} \frac{1}{n ! q !}\left(\frac{\bar{\gamma}}{\bar{\gamma}+m}\right)^{m+n} \gamma\left(m+n, \gamma_{T}\left(\frac{\bar{\gamma}+m}{\bar{\gamma}}\right)\right) .\right]
\end{aligned}
$$

Note that for $m=1,(6.15)$ reduces to dual Rayleigh correlated fading branches, and for $\rho=0$ reduces to dual i.i.d. Nakagami- $m$ fading branches detection.

\subsection{Alternative $\bar{P}_{D, S S C, 2}$ Expression}

The expression $\bar{P}_{D, S S C, 2}$ in (6.15) involves many infinite series representations. Some of their upper bounds (number of terms) are dependent on the preceded one. As an example, the upper bound of the second sum $\left(\sum_{k=0}^{j+u-1}().\right)$ depends on the number of terms $(N)$ needed for convergence of the previous series. Consequently, it will not be very difficult to find the number of terms for convergence (with five digit accuracy). However, time for numerical implementation will be rather long. Therefore, we will 
derive an alternative more general and simpler expression $\bar{P}_{D, S S C, 2}$ with less number of infinite series representations within the expression.

\subsubsection{Evaluation the integral $I_{A}$ in (6.3)}

Let $x=\sqrt{2 \gamma}$, we rewrite $I_{A}$ in $(6.3)$ as

$$
I_{A}=\frac{4}{2^{-m}} \int_{0}^{\infty} Q_{u}(x, \sqrt{\lambda}) x^{m-1} \exp \left(-\frac{m x^{2}}{2 \bar{\gamma}}\right) \mathrm{d} x
$$

Using the following integral solution given by [82] as

$$
2 \int_{0}^{\infty} Q_{u}(\alpha x, \beta) x^{2 m-1} e^{-p x^{2}} \mathrm{~d} x=2 \sum_{j=0}^{\infty} \frac{\alpha^{2 j} 2^{m} \Gamma(m+j) \Gamma\left(u+j, \frac{\beta^{2}}{2}\right)}{\Gamma(u+j)\left(\alpha^{2}+2 p\right)^{m+j} j !}
$$

hence, the solution of (6.16) for real $u$-values is given as [87]

$$
I_{A}=4 \sum_{j=0}^{\infty} \frac{\Gamma(m+j) \Gamma\left(u+j, \frac{\lambda}{2}\right)}{\Gamma(u+j)\left(1+\frac{m}{\bar{\gamma}}\right)^{m+j} j !}
$$

\subsubsection{Evaluation the integral $I_{B}$ in (6.3)}

Similarly, using (3.19) and (5.13) for $Q_{u}(\sqrt{2 \gamma}, \sqrt{\lambda})$ and $Q_{m}\left(\sqrt{2 a \rho \gamma}, \sqrt{2 a \gamma_{T}}\right)$ respectively, with some simplification and rearranging, (6.8) becomes

$$
I_{B}=\sum_{n=0}^{\infty} \sum_{i=0}^{\infty} \sum_{k=0}^{i+m-1} \frac{\Gamma\left(u+n, \frac{\lambda}{2}\right)}{\Gamma(u+n) n !} \frac{a^{i+k} \rho^{i}}{i ! k !} \gamma_{T}^{k} e^{-a \gamma_{T}} \int_{0}^{\infty} \gamma^{m+n+i-1} e^{-\gamma\left(\frac{m}{\bar{\gamma}}+a \rho+1\right)} \mathrm{d} \gamma
$$


Using (5.16) and satisfying the condition, the solution of (6.19) can be written as [87]

$$
I_{B}=\sum_{n=0}^{\infty} \sum_{i=0}^{\infty} \sum_{k=0}^{i+m-1} \frac{\Gamma\left(u+n, \frac{\lambda}{2}\right) \Gamma(m+n+i) a^{i+k} \rho^{i}}{\Gamma(u+n)\left(\frac{m}{\gamma}+a \rho+1\right)^{m+n+i} n ! i ! k !} \gamma_{T}^{k} e^{-a \gamma_{T}}
$$

\subsubsection{Evaluation the integral $I_{C}$ in (6.3)}

Using (3.19) with some simplification, (6.11) becomes

$$
I_{C}=\sum_{p=0}^{\infty} \frac{\Gamma\left(u+p, \frac{\lambda}{2}\right)}{\Gamma(u+p) p !} \int_{0}^{\gamma_{T}} \gamma^{m+p-1} e^{-\gamma\left(\frac{\bar{\gamma}+m}{\bar{\gamma}}\right)} \mathrm{d} \gamma
$$

Using (6.13) and satisfying the condition therein with some simplification, hence, for $m \geq 1$ the solution of $(6.21)$ is [87]

$$
I_{C}=\sum_{p=0}^{\infty} \frac{\Gamma\left(u+p, \frac{\lambda}{2}\right)}{\Gamma(u+p) p !}\left(\frac{\bar{\gamma}+m}{\bar{\gamma}}\right)^{-(m+p)} \gamma\left(m+p, \gamma_{T}\left(\frac{\bar{\gamma}+m}{\bar{\gamma}}\right)\right)
$$

Substituting (6.18), (6.20) and (6.22) into (6.3), hence, the average detection probability for $u$ is not restricted while $(m \geq 1)$ is restricted to integer values as [87]

$$
\begin{array}{r}
\bar{P}_{D, S S C, 2}=\frac{1}{\Gamma(m)}\left(\frac{m}{\bar{\gamma}}\right)^{m}\left[4 \sum_{j=0}^{\infty} \frac{\Gamma\left(u+j, \frac{\lambda}{2}\right) \Gamma(m+j)}{\Gamma(u+j)\left(1+\frac{m}{\bar{\gamma}}\right)^{m+j} j !}\right. \\
-\sum_{n=0}^{\infty} \sum_{i=0}^{\infty} \sum_{k=0}^{i+m-1} \frac{\Gamma\left(u+n, \frac{\lambda}{2}\right) \Gamma(m+n+i) \gamma_{T}^{k} e^{-a \gamma_{T}} a^{i+k} \rho^{i}}{\Gamma(u+n)\left(\frac{m}{\gamma}+a \rho+1\right)^{m+n+i} n ! i ! k !} \\
\left.-\sum_{p=0}^{\infty} \frac{\Gamma\left(u+p, \frac{\lambda}{2}\right)}{\Gamma(u+p) p !}\left(\frac{\bar{\gamma}+m}{\bar{\gamma}}\right)^{-(m+p)} \gamma\left(m+p, \gamma_{T}\left(\frac{\bar{\gamma}+m}{\bar{\gamma}}\right)\right)\right] .
\end{array}
$$


Note, for $m=1$, (6.23) reduces to dual Rayleigh correlated fading branches, and for $\rho=0$ it reduces to dual i.i.d. Nakagami- $m$ fading branches detection.

Interestingly, the three terms in (6.23) contain the upper incomplete gamma function in addition to the lower incomplete gamma function in the last term. In fact, we can represent both these functions by the monotonically decreasing confluent hypergeometric function using [[75], Eq. (6.5.12)] and [[90], Eq. (1.6)) for lower and upper incomplete gamma functions, respectively. Consequently the infinite series terms in (6.23) converges rapidly.

\subsection{Optimum Threshold $\left(\gamma_{T}^{*}\right)$}

Optimum threshold could be obtained by differentiating $\bar{P}_{D, S S C, 2}$ in (6.3) with respect to $\gamma_{T}$ and solving $\frac{\partial}{\partial \gamma_{T}^{*}} \bar{P}_{D, S S C, 2}=0$ for $\gamma_{T}^{*}$ as follows.

Employing Leibniz's rule [[75], Eq. (3.3.7)] with the aid of following identity given in [ [81], Eq. (9)] as

$$
\frac{\partial}{\partial \beta} Q_{u}(\alpha, \beta)=-\beta\left(\frac{\beta}{\alpha}\right)^{u-1} \exp \left(-\frac{\alpha^{2}+\beta^{2}}{2}\right) I_{u-1}(\alpha \beta),
$$


hence, (6.3) becomes

$$
\begin{array}{r}
\frac{\partial}{\partial \gamma_{T}^{*}} \bar{P}_{D, S S C, 2}=\frac{1}{\Gamma(m)}\left(\frac{m}{\bar{\gamma}}\right)^{m}\left[\rho^{\frac{1-m}{2}} \sqrt{2 a} \gamma_{T}^{* 1-\frac{m}{2}} e^{-a \gamma_{T}^{*}}\right. \\
\times \underbrace{\int_{0}^{\infty} Q_{u}(\sqrt{2 \gamma}, \sqrt{\lambda}) \gamma^{\frac{m-1}{2}} e^{-a \gamma} I_{m-1}\left(2 a \sqrt{\rho \gamma_{T}^{*} \gamma}\right) \mathrm{d} \gamma}_{I} \\
\left.-Q_{u}\left(\sqrt{2 \gamma_{T}^{*}}, \sqrt{\lambda}\right) \gamma_{T}^{* m-1} \exp \left(-\frac{m \gamma_{T}^{*}}{\bar{\gamma}}\right)\right] .
\end{array}
$$

To simplify solving the integral $I$ in (6.25), we perform changing variable along with the aid of the series expansion of the modified Bessel function given in [[77], Eq. $(8.445)]$ as

$$
I_{\nu}(z)=\sum_{k=0}^{\infty} \frac{1}{\Gamma(\nu+k+1) k !}\left(\frac{z}{2}\right)^{\nu+2 k} .
$$

Then, after lengthy simplification (6.25) becomes

$$
\begin{gathered}
\frac{\partial}{\partial \gamma_{T}^{*}} \bar{P}_{D, S S C, 2}=\frac{1}{\Gamma(m)}\left(\frac{m}{\bar{\gamma}}\right)^{m}\left[\rho^{\frac{1-m}{2}} \sqrt{2 a \gamma_{T}^{*}} e^{-a \gamma_{T}^{*}} \sum_{k=0}^{\infty} \frac{1}{\Gamma(m+k) 2^{m+k} k !}(a \sqrt{\rho})^{m+2 k-1} \gamma_{T}^{* k}\right. \\
\times \underbrace{\int_{0}^{\infty} Q_{u}(x, \sqrt{\lambda}) x^{2(m+k)-1} e^{-\frac{a}{2} x^{2}} \mathrm{~d} x}_{I}-Q_{u}\left(\sqrt{2 \gamma_{T}^{*}}, \sqrt{\lambda}\right) \gamma_{T}^{* m-1} \exp \left(-\frac{m \gamma_{T}^{*}}{\bar{\gamma}}\right)] .
\end{gathered}
$$

Using [[81], Eq. (29)] by following same procedures as in (5.9), we can solve the integral $I$ in (6.27) as

$$
I_{A}=G_{1}^{\prime}+\frac{1}{2} \sum_{n=1}^{u-1}\left(\frac{\lambda}{2}\right)^{n} \frac{\Gamma(m+k)}{\left(\frac{a+1}{2}\right)^{m+k} n !}{ }_{1} F_{1}\left(m+k ; n+1 ; \frac{\lambda}{2(a+1)}\right) \text {, }
$$


where $G_{1}^{\prime}$ can be obtained by evaluating the following integral containing the first order of Marcum $Q$-function $Q(.,$.$) for integer m$ values as

$$
G_{1}^{\prime}=\int_{0}^{\infty} Q(x, \sqrt{\lambda}) x^{2(m+k)-1} e^{-\frac{a}{2} x^{2}} \mathrm{~d} x .
$$

Using [[81], Eq. (25)], we evaluate $G_{1}^{\prime}$ for integer $m$ values as

$$
\begin{aligned}
& G_{1}^{\prime}=\frac{2^{m+k-1}(m+k-1) !}{a^{2(m+k)}}\left(\frac{1}{a+1}\right) e^{-\frac{\lambda}{2} \frac{a}{a+1}} \\
& \times\left[(1+a)\left(\frac{a}{1+a}\right)^{m+k-1} L_{m+k-1}\left(-\frac{\lambda}{2(1+a)}\right)+\sum_{n=0}^{m+k-2}\left(\frac{a}{a+1}\right)^{n} L_{n}\left(-\frac{\lambda}{2(a+1)}\right)\right] .
\end{aligned}
$$

Here $L_{n}($.$) denotes Laguerre polynomial of n$-degree [77].

Substituting (6.28) into (6.27) with simplifying, yields [87]

$$
\begin{array}{r}
\frac{\partial}{\partial \gamma_{T}^{*}} \bar{P}_{D, S S C, 2}=\frac{1}{\Gamma(m)}\left(\frac{m}{\bar{\gamma}}\right)^{m}\left[\sqrt{2 a \gamma_{T}^{*}} e^{-a \gamma_{T}^{*}} \sum_{k=0}^{\infty} \frac{a^{m+2 k-1} \rho^{k}}{\Gamma(m+k) 2^{m+k} k !} \gamma_{T}^{* k}\right. \\
\times\left\{G_{1}^{\prime}+\frac{1}{2} \sum_{n=1}^{u-1}\left(\frac{\lambda}{2}\right)^{n} \frac{\Gamma(m+k)}{\left(\frac{a+1}{2}\right)^{m+k} n !} F_{1}\left(m+k ; n+1 ; \frac{\lambda}{2(a+1)}\right)\right\} \\
\left.-Q_{u}\left(\sqrt{2 \gamma_{T}^{*}}, \sqrt{\lambda}\right) \gamma_{T}^{* m-1} \exp \left(-\frac{m \gamma_{T}^{*}}{\bar{\gamma}}\right)\right] .
\end{array}
$$

Finally, by solving $\frac{\partial}{\partial \gamma_{T}^{*}} \bar{P}_{D, S S C, 2}=0$ for $\gamma_{T}^{*}$ numerically in (6.31), we can obtain the optimum threshold. 


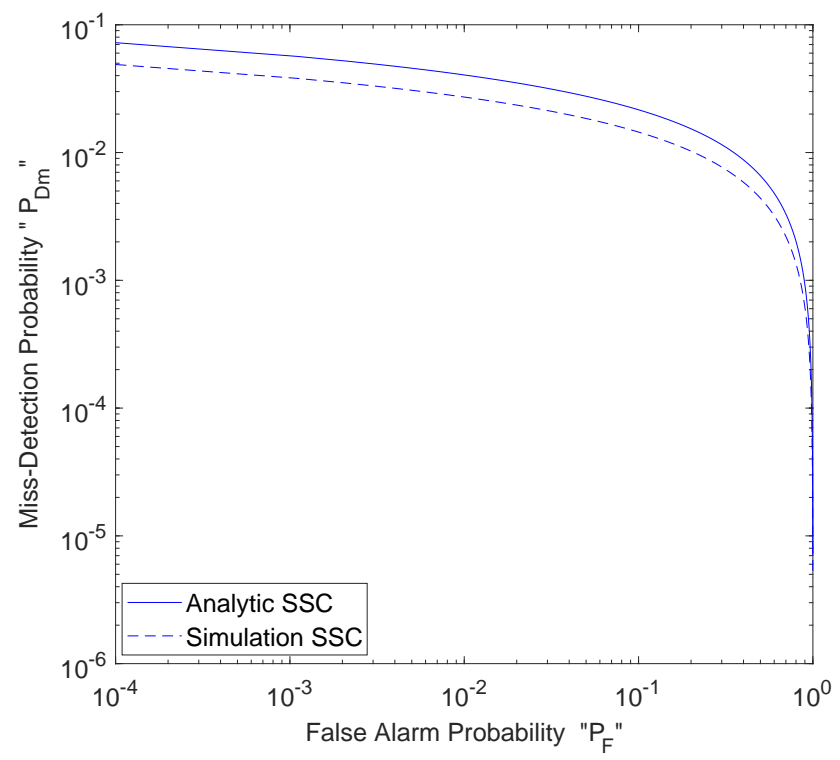

Figure 6.2: Analytic (solid) versus simulation (dashed) results for SSC with $L=$ $2, m=1, \bar{\gamma}=20 \mathrm{~dB}$ and $\rho=0.8$.

\subsection{Performance Evaluation}

In this section, we verify the derived closed-form expressions for different cases of interest for spectrum sensing model with SSC technique. The impact of multipath fading and correlation on average detection probability $\bar{P}_{D}$ (as a performance factor) is investigated.

We follow same procedures as we did in Sec. 3.4 to calculate the corresponding threshold for the assumed detection parameters to produce corresponding SSC' CROC graphs. In Figure. 6.2, numerical results obtained for analytic and simulation are almost in a good match. However, reader may observe a very small difference between analytic and simulation curves. This is due to the inaccuracy arising from rounding off the infinite series and optimum threshold calculation. 
In Figure 6.3, and for each value of fading severity $m$, one can notice clearly the degradation in detection probability due to the correlation among diversity branches. However, as $m$ increases, correlation effect is compensated, resulting in higher detection probability. Similar observations as in SC diversity technique in Sec. 5.4 are obtained here for SSC. Therefore, we can conclude that for deep fading environment, correlation must be considered for accurate cognitive detection, while for low fading environment, its effect may be ignored. 


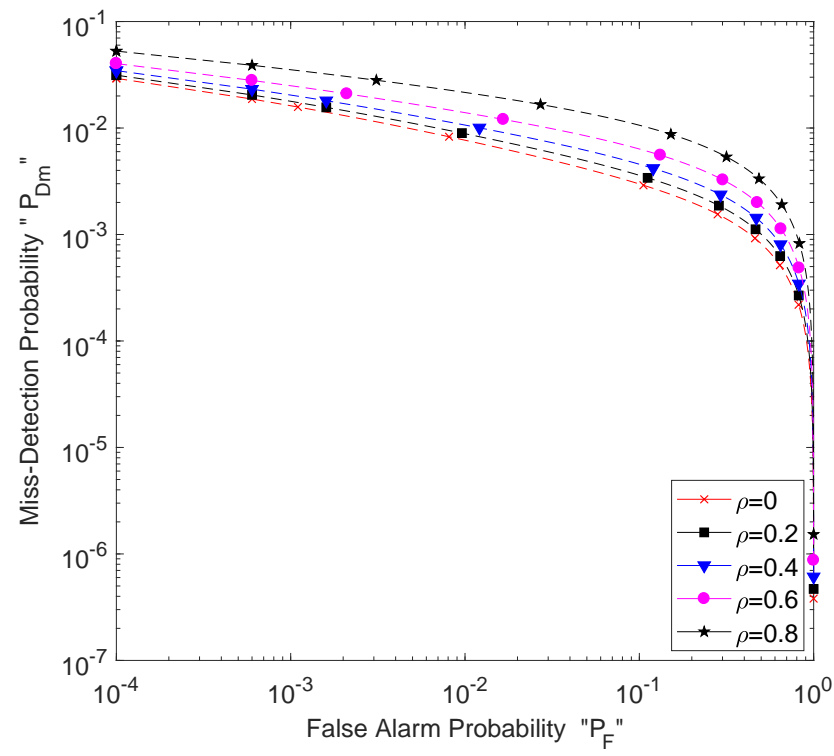

(a) $m=1$ (Rayleigh: deep fading)

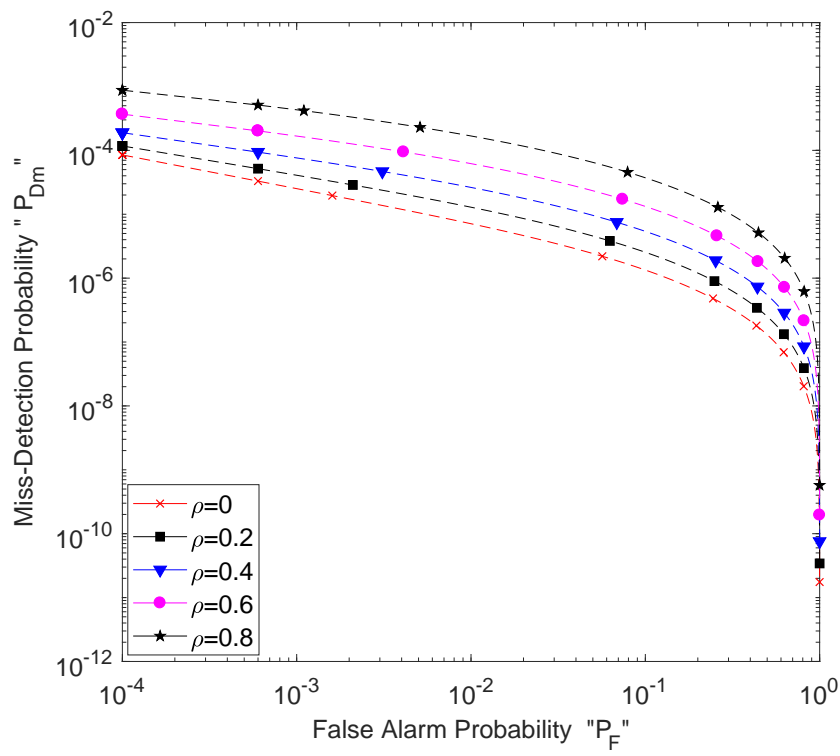

(b) $m=4$ (Low fading)

Figure 6.3: Dual correlated Nakagami- $m$ branches with SSC diversity; $\bar{\gamma}=20 \mathrm{~dB}$, $L=2$ and for different $\rho$ values. 


\section{Chapter 7}

\section{Decorrelation-Based SC Receiver in Nakagami-m Fading Channel}

Multipath fading and correlation among reception branches are of a great source of sensing impairment due to the nature of the signal propagating medium and insufficient spacing antenna spacing in small-size mobile unites. Consequently, assuming independent branches is invalid for many real sensing scenarios. Employing diversity technique to maximize the SNR helps mitigate the destructive effect of the multipath fading. However, maximal theoretical diversity gain is unattainable [18] with correlated branches. Thus, it is important to combat the correlation impact on diversity performance. 


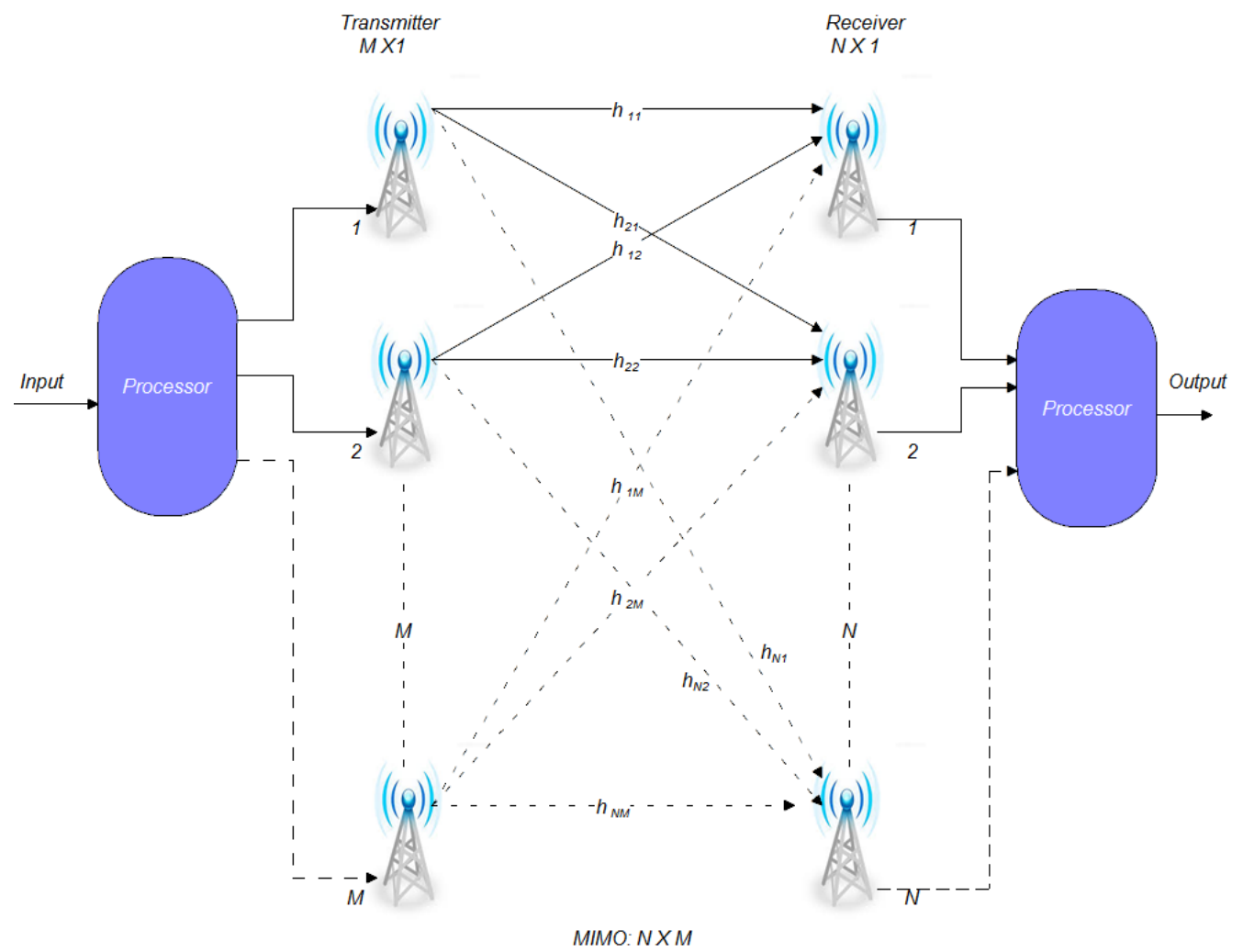

Figure 7.1: MIMO system.

Decorrelation is one of the techniques employed to mitigate effectively the correlation adverse impact in wireless communications. Covariance matrix transformation such as KLT $[20,91]$ is often employed in this technique, consequently, the knowledge of the covariance matrix is necessary to perform the diagonalization [20].

In general, the received observation vector for MIMO shown in Figure 7.1 is given as

$$
\mathbf{x}=\mathbf{H s}+\mathbf{n},
$$

where $\mathbf{H}(N \times M), \mathbf{s}(M \times 1)$ and $\mathbf{n}(N \times 1)$ denote channel matrix, received signals vector and noise vector, respectively. Hence, for $K$ correlated observations from the 
model (7.1) to $N \times K$ matrix $\mathbf{X}=\left[\mathbf{x}_{1}, \cdots, \mathbf{x}_{K}\right]$, the corresponding covariance matrix $\mathbf{R}_{\mathbf{X}}=\mathrm{E}\left[\mathbf{X X}^{\mathrm{T}}\right]^{1}$ is $N \times N$ symmetric matrix with diagonal elements represent the variances and the off-diagonal elements represent the covariances. For i.i.d. random variables, the off-diagonal elements are zeros and $\mathbf{R}_{\mathbf{X}}$ becomes diagonal matrix.

To diagonalize $\mathbf{R}_{\mathbf{X}}$, there is a matrix $\mathbf{P}$ satisfies [20]

$$
\mathbf{P}^{\mathrm{T}} \mathbf{R}_{\mathbf{X}} \mathbf{P}=\Lambda, \quad \mathbf{P}^{\mathrm{T}} \mathbf{P}=\mathbf{I},
$$

where $\Lambda$ is a diagonal matrix and $\mathbf{I}$ is the identity matrix. KLT is often employed to find $\mathbf{P}$ for this diagonalization.

In some special cases where dual diversity is employed, decorrelation can be performed easily by adding and subtracting the diversity branches. However, this requires the evaluation of the statistics of the sum and the difference of correlated bivariate, due to the random nature of the wireless channel.

In this chapter, we propose a decorrelated-based SC receiver. We do so through investigating the problem of decorrelating SC receiver in identical and correlated dual Nakagami- $m$ fading branches. We derive the expressions for statistics of the normalized difference of Nakagami- $m$ bivariate which represent the foundation of our presented decorrelator. To the best of our knowledge, we believe that these derived expressions are novel in the literature.

\footnotetext{
${ }^{1} \mathrm{~T}$ denotes transpose of a matrix
} 


\subsection{System Model}

Assuming dual identical and correlated antenna branches in Nakagami- $m$ channels, we can write

$$
\begin{aligned}
& x_{1}=r_{1} s+n_{1}, \\
& x_{2}=r_{2} s+n_{2},
\end{aligned}
$$

where, $s$ denotes the data signal samples, $r_{i}=\alpha_{i} e^{j \theta_{i}}, i=1,2$, is the slow varying complex channel gain coefficient with amplitude $\alpha_{i}$ and phase $\theta_{i}$ and, $n_{i}, i=1,2$, is the additive white Gaussian noise (AWGN) with zero mean and variance $N_{0} / 2$ per dimension. The instantaneous and average SNR are denoted by $\gamma$ and $\bar{\gamma}$, respectively. Following [74], the corresponding PDF of a univariate $\alpha$-Nakagami- $m$ variable is given by

$$
p_{\alpha}(\alpha)=\frac{2 \alpha^{2 m-1}}{\Gamma(m)}\left(\frac{m}{\Omega}\right)^{m} e^{-\frac{m \alpha^{2}}{\Omega}}, \quad \alpha \geq 0,
$$

where, $\Gamma($.$) denotes the Gamma function, \Omega=E\left[\alpha^{2}\right] / m=\frac{\overline{\alpha^{2}}}{m}$ is the mean-square value of the variable $\alpha$ and $m(m \geq 1 / 2)$ is the inverse normalized variance of $\alpha^{2}$ which describes the fading severity. The envelope $\alpha$ of the Nakagami- $m$ distribution could be represented by the square root of the sum of squares of $\mathrm{Lm}$ independent complex Gaussian variates $[74,88,92]$ given as

$$
g_{k i}=\left(\sqrt{1-\rho} X_{k i}+\sqrt{\rho} X_{0 i}\right)+j\left(\sqrt{1-\rho} Y_{k i}+\sqrt{\rho} Y_{0 i}\right)
$$


where, $L$ is the number of paths, $k=1, \cdots, L ; i=1, \cdots, m ; 0 \leq \rho \leq 1 ; j=\sqrt{-1}$ and; $X_{k i}$ and $Y_{k i} \sim N\left(0, \frac{1}{2}\right), k=0, \cdots, L ; i=1, \cdots, m$ are independent Gaussian random variables.

Therefore, the summation of the absolute squares of $g_{k i}$ is given as

$$
G_{k}=\sum_{i=1}^{m}\left|g_{k i}\right|^{2} .
$$

Consequently, $\alpha_{k}=\sqrt{G_{k}}$ is a set of identically and equally correlated Nakagami- $m$ fading envelopes. The decorrelator outputs are $U_{1}$ and $U_{2}$ respectively as

$$
\begin{aligned}
& U_{1}=\frac{x_{1}+x_{2}}{\sqrt{2}}=\frac{r_{1}+r_{2}}{\sqrt{2}} s+\frac{n_{1}+n_{2}}{\sqrt{2}}=\sigma s+\nu_{1}, \\
& U_{2}=\frac{x_{1}-x_{2}}{\sqrt{2}}=\frac{r_{1}-r_{2}}{\sqrt{2}} s+\frac{n_{1}-n_{2}}{\sqrt{2}}=\delta s+\nu_{2},
\end{aligned}
$$

where $\sigma$ and $\delta$ are two random variables denote the normalized sum and normalized difference of the correlated branches, respectively. Furthermore it is easy to show that $\sigma$ and $\delta$ are uncorrelated. Terms $\nu_{1}$ and $\nu_{2}$ represent the noise which is also easy to show they are mutually independent Gaussian random variables with zero mean, and unity variance.

We are more interested in $U_{2}$, which is the difference. Next, we will evaluate the PDF and CDF of the normalized difference $\delta$ in (7.9) which represent the foundation of our proposed decorrelator. 


\subsection{Statistics of Normalized Difference of Identical and correlated Nakagami- $m$ Bivariate: $\delta=\frac{r_{1}-r_{2}}{\sqrt{2}}$}

\subsubsection{PDF's Expression: $f_{\Delta}(\delta)$}

Let $r_{1}, r_{2} \geq 0$ be Nakagami- $m$ correlated bivariate with $p_{R 1 R 2}\left(r_{1}, \Omega_{1}, r_{2}, \Omega_{2} \mid m, \rho\right)$ denoted as the joint PDF. By definition, the CDF of the difference $\delta=\frac{r_{1}-r_{2}}{\sqrt{2}}$ is $F_{\Delta}(\delta)=P\left(\frac{r_{1}-r_{2}}{\sqrt{2}} \leq \delta\right)$, hence

$$
F_{\Delta}(\delta)=\int_{0}^{\infty} \int_{0}^{\sqrt{2} \delta+r_{2}} f_{R_{1} R_{2}}\left(r_{1}, r_{2}\right) \mathrm{d} r_{1} \mathrm{~d} r_{2}
$$

where $f_{R_{1} R_{2}}\left(r_{1}, r_{2}\right)$ denotes the joint pdf. Due to the complexity of solving the double integral in (7.10) analytically for $\delta \neq$, we will evaluate the corresponding PDF as follows.

Applying Leibniz's theorem of differentiation of an integral rule given in [[75], Eq. $(3.3 .7)]$ as

$$
\frac{\mathrm{d}}{\mathrm{d} c} \int_{a(c)}^{b(c)} f(x, c) d x=\int_{a(z)}^{b(c)} \frac{\partial}{\partial c} f(x, c) d x+f(b, c) \frac{\mathrm{d} b}{\mathrm{~d} c}-f(a, c) \frac{\mathrm{d} a}{\mathrm{~d} c},
$$

the corresponding PDF could be obtained by differentiating (7.10) yields

$$
f_{\Delta}(\delta)=\sqrt{2} \int_{0}^{\infty} f_{R_{1} R_{2}}\left(\sqrt{2} \delta+r_{2}, r_{2}\right) \mathrm{d} r_{2} .
$$


We must consider separately the following two cases

$$
f_{\Delta}(\delta)= \begin{cases}\sqrt{2} \int_{0}^{\infty} f_{R_{1} R_{2}}\left(r_{2}, r_{2}\right) \mathrm{d} r_{2} & \delta=0 . \\ \sqrt{2} \int_{0}^{\infty} f_{R_{1} R_{2}}\left(\sqrt{2} \delta+r_{2}, r_{2}\right) \mathrm{d} r_{2} & \delta \neq 0 .\end{cases}
$$

For correlated Nakagami- $m$ bivariate $r_{1}$ and $r_{2}$, their joint PDF is given by [[19], Eq. (6.1)] as

$$
\begin{gathered}
f_{R 1 R 2}\left(r_{1}, r_{2}\right)=\frac{4 m^{m+1}}{\Gamma(m) \Omega_{1} \Omega_{2}(1-\rho)\left(\sqrt{\Omega_{1} \Omega_{2} \rho}\right)^{m-1}} \\
\times\left(r_{1} r_{2}\right)^{m} \exp \left[-\frac{m}{1-\rho}\left(\frac{r_{1}^{2}}{\Omega_{1}}+\frac{r_{2}^{2}}{\Omega_{2}}\right)\right] I_{m-1}\left(\frac{2 m \sqrt{\rho} r_{1} r_{2}}{\sqrt{\Omega_{1} \Omega_{2}}(1-\rho)}\right), \quad r_{1}, r_{2} \geq 0,
\end{gathered}
$$

where $I_{\nu}($.$) denotes modified Bessel function of \nu$-kind and $\rho=\frac{\operatorname{cov}\left(r_{1}^{2}, r_{2}^{2}\right)}{\sqrt{\left(\operatorname{var}\left(r_{1}^{2}\right) \operatorname{var}\left(r_{2}^{2}\right)\right)}}$ denotes the power correlation coefficient $(0 \leq \rho<1)$.

Using the series expansion of the modified Bessel function in (6.26) with some simplification and rearranging, hence, for identical bivariate $\left(\Omega_{1}=\Omega_{2}=\Omega\right)$, (7.14) becomes

$$
\begin{aligned}
f_{R 1 R 2}\left(r_{1}, r_{2}\right)= & \frac{4}{\Gamma(m)} \sum_{k=0}^{\infty} \frac{\rho^{k}}{\Gamma(m+k)(1-\rho)^{m+2 k} k !}\left(\frac{m}{\Omega}\right)^{2(m+k)} \\
& \times\left(r_{1} r_{2}\right)^{2(m+k)-1} \exp \left[-\frac{m}{\Omega(1-\rho)}\left(r_{1}^{2}+r_{2}^{2}\right)\right] .
\end{aligned}
$$

Substituting $r_{1}=\sqrt{2} \delta+r_{2}$ into (7.15), we can write the joint PDF of normalized 
difference of identical and correlated Nakagami bivariate as

$$
\begin{gathered}
f_{R 1 R 2}\left(r_{1}, r_{2}\right) \frac{4}{\Gamma(m)} \sum_{k=0}^{\infty} \frac{\rho^{k}}{\Gamma(m+k)(1-\rho)^{m+2 k} k !}\left(\frac{m}{\Omega}\right)^{2(m+k)} \\
\times\left[r_{2}\left(\sqrt{2} \delta+r_{2}\right)\right]^{2(m+k)-1} \exp \left[-\frac{m}{\Omega(1-\rho)}\left\{\left(\sqrt{2} \delta+r_{2}\right)^{2}+r_{2}^{2}\right\}\right] .
\end{gathered}
$$

Therefore, the corresponding PDF could be obtained by substituting (7.16) into (7.12) yields

$$
\begin{array}{r}
f_{\Delta}(\delta)=\frac{4 \sqrt{2}}{\Gamma(m)} \sum_{k=0}^{\infty} \frac{\rho^{k}}{\Gamma(m+k)(1-\rho)^{m+2 k} k !}\left(\frac{m}{\Omega}\right)^{2(m+k)} \\
\times \int_{0}^{\infty}\left[r_{2}\left(\sqrt{2} \delta+r_{2}\right)\right]^{2(m+k)-1} \exp \left[-\frac{m}{\Omega(1-\rho)}\left\{\left(\sqrt{2} \delta+r_{2}\right)^{2}+r_{2}^{2}\right\}\right] d r_{2} .
\end{array}
$$

As we pointed out, we will solve (7.17) for the following two cases given in (7.13).

A. Case 1: $\delta=0$

Substituting $\delta=0$ into (7.17), changing variables and using [[77], Eq. (3.381/4)] given as

$$
\int_{0}^{\infty} x^{\nu-1} e^{-\mu x} \mathrm{~d} x=\frac{1}{\mu^{\nu}} \Gamma(\nu) \quad \Re \mu>0, \Re \nu>0
$$

and satisfying the conditions therein with some simplification, the PDF for $\delta=0$ is given as [93]

$$
f_{\Delta}(0)=\frac{4(1-\rho)^{m}}{\Gamma(m)}\left(\frac{m}{\Omega(1-\rho)}\right)^{\frac{1}{2}} \sum_{k=0}^{\infty} \frac{\Gamma\left[2(m+k)-\frac{1}{2}\right] \rho^{k}}{\Gamma(m+k) 2^{2(m+k)} k !} .
$$


B. Case 2: $\delta \neq 0$

With some simplification and mathematical manipulation, (7.17) becomes

$$
\begin{array}{r}
f_{\Delta}(\delta)=\frac{4 \sqrt{2}}{\Gamma(m)} \sum_{k=0}^{\infty} \frac{\rho^{k}}{\Gamma(m+k)(1-\rho)^{m+2 k} k !}\left(\frac{m}{\Omega}\right)^{2(m+k)} \\
\times e^{-\frac{m}{\Omega(1-\rho)} \delta^{2}} \int_{0}^{\infty}\left[r_{2}\left(\sqrt{2} \delta+r_{2}\right)\right]^{2(m+k)-1} \exp \left[-\frac{m}{\Omega(1-\rho)}\left(\sqrt{2} r_{2}+\delta\right)^{2}\right] d r_{2} .
\end{array}
$$

Using changing variable, $y=\frac{m}{\Omega(1-\rho)}\left(\sqrt{2} r_{2}+\delta\right)^{2}$ and modifying the integral limits with some math work and simplification yields

$$
\begin{aligned}
f_{\Delta}(\delta)=\frac{2}{\Gamma(m)} e^{-\frac{m}{\Omega(1-\rho)} \delta^{2}} \sum_{k=0}^{\infty} \frac{\rho^{k}(1-\rho)^{m}}{\Gamma(m+k) 2^{2(m+k)-1} k !}\left(\frac{m}{\Omega(1-\rho)}\right)^{\frac{1}{2}} \\
\times \underbrace{\int_{\frac{m}{\Omega(1-\rho)} \delta^{2}}^{\infty} y^{-\frac{1}{2}}\left[y-\frac{m}{\Omega(1-\rho)} \delta^{2}\right]^{2(m+k)-1} e^{-y} d y}_{I}
\end{aligned}
$$

We solve (7.21) by evaluating the Integral $I$ using [[77], Eq. (3.383/4)] given as

$$
\begin{array}{r}
\int_{u}^{\infty} x^{\nu-1}(x-u)^{\mu-1} e^{-\beta x} d x=\beta^{-\frac{(\mu+\nu)}{2}} u^{\frac{\mu+\nu-2}{2}} \Gamma(\mu) e^{-\frac{\beta u}{2}} W_{\frac{\nu-\mu}{2}}, \frac{1-\mu-\nu}{2}(\beta u), \\
{[\Re \mu>0, \Re \beta u>0],}
\end{array}
$$

where $W_{a, b}(z)$ denotes Whittaker function. Satisfying the conditions therein, hence, the PDF of normalized difference of identical and correlated Nakagami bivariate for 
$\delta \neq 0$ and not restricted to $m$ integer values could be written as [93]

$$
\begin{aligned}
f_{\Delta}(\delta)= & \frac{4(1-\rho)^{m}}{\Gamma(m)} \sum_{k=0}^{\infty} \frac{\Gamma[2(m+k)] \rho^{k}}{\Gamma(m+k) 2^{2(m+k)-1} k !}\left[\frac{m}{\Omega(1-\rho)}\right]^{m+k-\frac{1}{4}} \\
& \times \delta^{2(m+k)-\frac{3}{2}} e^{-\frac{3}{2} \frac{m}{\Omega(1-\rho)} \delta^{2}} W_{\frac{1}{4}-(m+k), \frac{1}{4}-(m+k)}\left(\frac{m}{\Omega(1-\rho)} \delta^{2}\right) .
\end{aligned}
$$

\subsubsection{Alternative PDF's Expression}

In this section, we provide an alternative and simpler PDF expression $f_{\Delta}(\delta)$ to that in (7.23) which is applicable only integer and half-odd integer values of $m$.

Using [[77], Eq. (1.111)] given as

$$
(a+x)^{\beta}=\sum_{l=0}^{\beta}\left(\begin{array}{l}
\beta \\
l
\end{array}\right) x^{l} a^{\beta-l}
$$

where $\left(\begin{array}{c}\beta \\ l\end{array}\right)=\frac{\beta !}{(\beta-l) ! l !}$ denotes the binomial coefficient, the term $\left[y-\frac{m}{\Omega(1-\rho)} \delta^{2}\right]^{2(m+k)-1}$ in (7.21) and for natural $\beta=2(m+k)-1$ values, could be expanded. Hence the integral part $I$ in $(7.21)$ becomes

$$
I=\sum_{l=0}^{\beta}\left(\begin{array}{c}
2(m+k)-1 \\
l
\end{array}\right)\left[-\frac{m}{\Omega(1-\rho)} \delta^{2}\right]^{l} \int_{\frac{m}{\Omega(1-\rho)} \delta^{2}}^{\infty} y^{2(m+k)-l-\frac{3}{2}} e^{-y} d y
$$

To solve (7.25), we use [[77], Eq. (3.381/3)] given as

$$
\int_{u}^{\infty} x^{\nu-1} e^{-\mu x} d x=\mu^{-\nu} \Gamma(\nu, \mu u), \quad[\Re \nu>0, u>0]
$$


and satisfying the conditions therein yields

$I=\sum_{l=0}^{2(m+k)-1}(-1)^{l}\left(\begin{array}{c}2(m+k)-1 \\ l\end{array}\right)\left(\frac{m}{\Omega(1-\rho)}\right)^{l} \delta^{2 l} \Gamma\left(2(m+k)-l-\frac{1}{2}, \frac{m \delta^{2}}{\Omega(1-\rho)}\right)$

where $\Gamma(a, x)$ denotes incomplete upper gamma function defined in [[77], Eq. (8.350/2)].

Substituting (7.27) into (7.21) with simplification and rearrangement, the PDF of normalized difference of identical and correlated Nakagami bivariate for integer and half-odd integer $m$ values as [93]

$$
\begin{aligned}
f_{\Delta}(\delta)=\frac{4(1-\rho)^{m}}{\Gamma(m)} \sum_{k=0}^{\infty} \sum_{l=0}^{2(m+k)-1}(-1)^{l} \frac{(2(m+k)-1) ! \rho^{k}}{\Gamma(m+k)(2(m+k)-l-1) ! 2^{2(m+k)} k ! l !} \\
\quad \times\left(\frac{m}{\Omega(1-\rho)}\right)^{l+\frac{1}{2}} \delta^{2 l} e^{-\frac{m}{\Omega(1-\rho)} \delta^{2}} \Gamma\left(2(m+k)-l-\frac{1}{2}, \frac{m}{\Omega(1-\rho)} \delta^{2}\right) .
\end{aligned}
$$

Note that both expressions in (7.23) and (7.28) converge rapidly as shown in Table 7.1. Also note that we derived the expression in (7.23) since it is a little bit more general ${ }^{2}$ than (7.28) in studying the PDF's behavior of the bivariate normalized difference. However, using it to derive the corresponding CDF, will result in an intractable and not simple closed-form expression due to the presence of the Whittaker function. Therefore, we will use the expression in (7.28) in this derivation.

\footnotetext{
${ }^{2}$ Not restricted to $m$ integer values
} 


\subsubsection{CDF Expression}

In this section, we will evaluate the corresponding CDF for normalized difference of identical and correlated Nakagami bivariate for the two investigated cases mentioned before.

Case I: $\delta=0$

By setting $\delta=0$ in (7.16) and substituting the result into (7.10), then solving the double integral by changing variables with some algebraic work and simplification yields [93]

$F_{\Delta}(0)=\frac{(1-\rho)^{m}}{\Gamma(m)} \sum_{k=0}^{\infty} \frac{\Gamma(2(m+k)) \rho^{k}}{\Gamma(m+k)(m+k) 2^{2(m+k)} k !}{ }^{2} F_{1}\left(1,2(m+k) ; m+k+1 ; \frac{1}{2}\right)$,

where ${ }_{2} F_{1}(a, b ; c ; z)$ denotes Gaussian Hypergeometric function defined in [[75], Eq. $(15.1 .1)]$

Case II: $\delta \neq 0$

By definition, the corresponding CDF could be obtained by integrating (7.28) as

$$
\begin{aligned}
F_{\Delta}(\delta) & =\frac{4(1-\rho)^{m}}{\Gamma(m)} \sum_{k=0}^{\infty} \sum_{l=0}^{2(m+k)-1}(-1)^{l} \frac{(2(m+k)-1) ! \rho^{k}}{\Gamma(m+k)(2(m+k)-l-1) ! 2^{2(m+k)} k ! l !} \\
& \times\left(\frac{m}{\Omega(1-\rho)}\right)^{l+\frac{1}{2}} \int_{0}^{\delta} \alpha^{2 l} e^{-\frac{m}{\Omega(1-\rho)} \alpha^{2}} \Gamma\left(2(m+k)-l-\frac{1}{2}, \frac{m}{\Omega(1-\rho)} \alpha^{2}\right) d \alpha
\end{aligned}
$$


To facilitate the solution of (7.30), we use the integral representation of the upper incomplete gamma function given by [[77], Eq. (8.353/5)] as

$$
\begin{array}{r}
\Gamma(a, x y)=y^{a} e^{-x y} \int_{0}^{\infty} e^{-t y}(t+x)^{(a-1)} d t, \\
{[\operatorname{Re}(y)>0, x>0, \operatorname{Re} a>1],}
\end{array}
$$

with multiple simplification and rearranging yields

$$
\begin{aligned}
& F_{\Delta}(\delta)=\frac{4(1-\rho)^{m}}{\Gamma(m)} \sum_{k=0}^{\infty} \sum_{l=0}^{2(m+k)-1}(-1)^{l} \frac{(2(m+k)-1) ! \rho^{k}}{\Gamma(m+k)(2(m+k)-l) ! 2^{2(m+k)} k ! l !} \\
& \times\left[\frac{m}{\Omega(1-\rho)}\right]^{2(m+k)} \int_{0}^{\infty}(t+1)^{2(m+k)-l-\frac{3}{2}} d t \underbrace{\int_{0}^{\delta} \alpha^{4(m+k)-1} e^{-\frac{m}{\Omega(1-\rho)} \alpha^{2}(t+2)} d \alpha}_{I} .
\end{aligned}
$$

To solve the integral $I$ in (7.32), we use [[77], Eq. (3.381/8)], hence, the CDF of the difference of identical and correlated Nakagami bivariate as [93]

$$
\begin{aligned}
& F_{\Delta}(\delta)=\frac{2(1-\rho)^{m}}{\Gamma(m)} \sum_{k=0}^{\infty} \sum_{l=0}^{2(m+k)-1}(-1)^{l} \frac{(2(m+k)-1) ! \rho^{k}}{\Gamma(m+k)(2(m+k)-l-1) ! 2^{2(m+k)} k ! l !} \\
& \times \int_{0}^{\infty}(t+1)^{2(m+k)-l-\frac{3}{2}}(t+2)^{-2(m+k)} \gamma\left(2(m+k), \frac{m}{\Omega(1-\rho)}(t+2) \delta^{2}\right) d t
\end{aligned}
$$

where $\gamma(a, x)$ denotes incomplete lower gamma function defined in [[77], Eq. (8.350/1)]. Note that it is easy to solve the above numerical integration using a commercial mathematical softwares such as Mathematica or Matlab. Furthermore, the infinite series converges rapidly as shown in Table 7.1, hence, it's not really worthwhile to simplify this integral. We'd like to point out that, in addition to decorrelation 
application, the derived expressions can also be used to solve the problem of self-interference in multicarrier systems. [94,95].

\subsection{The CDF of the Decorrelator}

Selection combining diversity technique is less complicated technique since the receiver selects the branch with the highest channel envelope value

$$
U=\max \left\{U_{l}, l=1,2, \ldots L\right\}
$$

As we stated in Sec. 7.1, the outputs of the decorrelator, $U_{1}$ and $U_{2}$, are independent that can be the input to the SC diversity combiner. Hence, for i.i.d. $U_{1}$ and $U_{2}$, the CDF of the combiner output is the product of their individual CDF's. That is

$$
F_{\text {Decor }}(\alpha)=F_{\Sigma}(\sigma) F_{\Delta}(\delta)
$$

where $\sigma$ denotes the sum of the normalized identical and correlated Nakagami- $m$ bivariate given in (7.8). The resulting CDF of the sum $\sigma$ is ${ }^{3}$

$$
\begin{array}{r}
F_{\Sigma}(\sigma)=\frac{4 \sqrt{\pi}(1-\rho)^{m}}{\Gamma(m)} \sum_{k=0}^{\infty} \sum_{n=0}^{\infty} \frac{\rho^{k}}{2^{4(m+k)+n} k ! n !} \\
\times \frac{\Gamma(2(m+k)+n)}{\Gamma(m+k) \Gamma\left(2(m+k)+n+\frac{1}{2}\right)} \gamma\left(2(m+k)+n, \frac{2 m \sigma^{2}}{\Omega(1-\rho)}\right) .
\end{array}
$$

Therefore, by changing variable $\delta^{2}=\Omega \gamma / \bar{\gamma}$ and $\sigma^{2}=\Omega \gamma / \bar{\gamma}$ in (7.33) and (7.36), respectively, and by substituting the results in (7.35), we derive the output CDF of

\footnotetext{
${ }^{3}$ Note, this is similar to the case where the output is of an EGC combiner for the same bivariate sum given in [[96], Eq. (4)].
} 
Table 7.1: Terms required for five digits accuracy

\begin{tabular}{|c|c|c|c|c|}
\hline & & $f_{\Delta}(\delta):\left|\tilde{E}_{N}\right|$ & $\Omega=1$ & \\
\hline$\rho$ & $\begin{array}{l}m=1 \\
N_{k}\end{array}$ & $\begin{array}{l}m=2 \\
N_{k}\end{array}$ & $\begin{array}{l}m=3 \\
N_{k}\end{array}$ & $\begin{array}{l}m=4 \\
N_{k}\end{array}$ \\
\hline 0 & 1 & 1 & 1 & 1 \\
\hline 0.3 & 4 & 6 & 8 & 10 \\
\hline 0.6 & 10 & 10 & 18 & 22 \\
\hline & & $F_{\Delta}(\delta): \tilde{E}_{N}$ & $\Omega=1$ & \\
\hline$\rho$ & $\begin{array}{l}m=1 \\
N_{k}\end{array}$ & $\begin{array}{l}m=2 \\
N_{k}\end{array}$ & $m=3$ & $\begin{array}{l}m=4 \\
N_{k}\end{array}$ \\
\hline 0 & 1 & 1 & 1 & 1 \\
\hline 0.3 & 8 & 8 & 10 & 10 \\
\hline 0.6 & 15 & 15 & 18 & 22 \\
\hline & & Decorr $(\gamma): \tilde{E}_{N}$ & $\bar{\gamma}=0 \mathrm{~dB}$ & \\
\hline$\rho$ & $\begin{array}{l}m=1 \\
N_{k_{1}}, N_{k_{2}}, N_{n}\end{array}$ & $\begin{array}{lr}m= & 2 \\
N_{k_{1}}, N_{k_{2}}, & N_{n}\end{array}$ & $\begin{array}{l}m=3 \\
N_{k_{1}}, N_{k_{2}}, N_{n}\end{array}$ & $\begin{array}{l}m=4 \\
N_{k_{1}}, N_{k_{2}}, N_{n}\end{array}$ \\
\hline 0 & $1,1,10$ & $1,1,15$ & $1,1,20$ & $1,1,25$ \\
\hline 0.3 & $10,8,15$ & $20,8,25$ & $20,10,25$ & $17,10,40$ \\
\hline 0.6 & $15,15,25$ & $20,15,40$ & $15,18,50$ & $17,22,60$ \\
\hline
\end{tabular}

the decorrelated receiver as [93]

$$
\begin{aligned}
& F_{\text {Decorr }}(\gamma)=\frac{8 \sqrt{\pi}(1-\rho)^{2 m}}{\Gamma^{2}(m)} \sum_{k_{1}=0}^{\infty} \sum_{n=0}^{\infty} \frac{\Gamma\left(2\left(m+k_{1}\right)+n\right) \rho^{k_{1}}}{\Gamma\left(m+k_{1}\right) \Gamma\left(2\left(m+k_{1}\right)+n+\frac{1}{2}\right) k_{1} ! n !} \\
& \times \sum_{k_{2}=0}^{\infty} \sum_{l=0}^{2\left(m+k_{2}\right)-1}(-1)^{l} \frac{\left(2\left(m+k_{2}\right)-1\right) ! \rho^{k_{2}}}{\Gamma\left(m+k_{2}\right)\left(2\left(m+k_{2}\right)-l-1\right) ! 2^{4\left(m+k_{1}\right)+2\left(m+k_{2}\right)+n} k_{2} ! l !} \\
& \times \gamma\left(2\left(m+k_{1}\right)+n, \frac{2 m}{\bar{\gamma}(1-\rho)} \gamma\right) \int_{0}^{\infty}(t+1)^{2\left(m+k_{2}\right)-l-\frac{3}{2}}(t+2)^{-2\left(m+k_{2}\right)} \\
& \times \gamma\left(2\left(m+k_{2}\right), \frac{m}{\bar{\gamma}(1-\rho)} \gamma(t+2)\right) d t .
\end{aligned}
$$




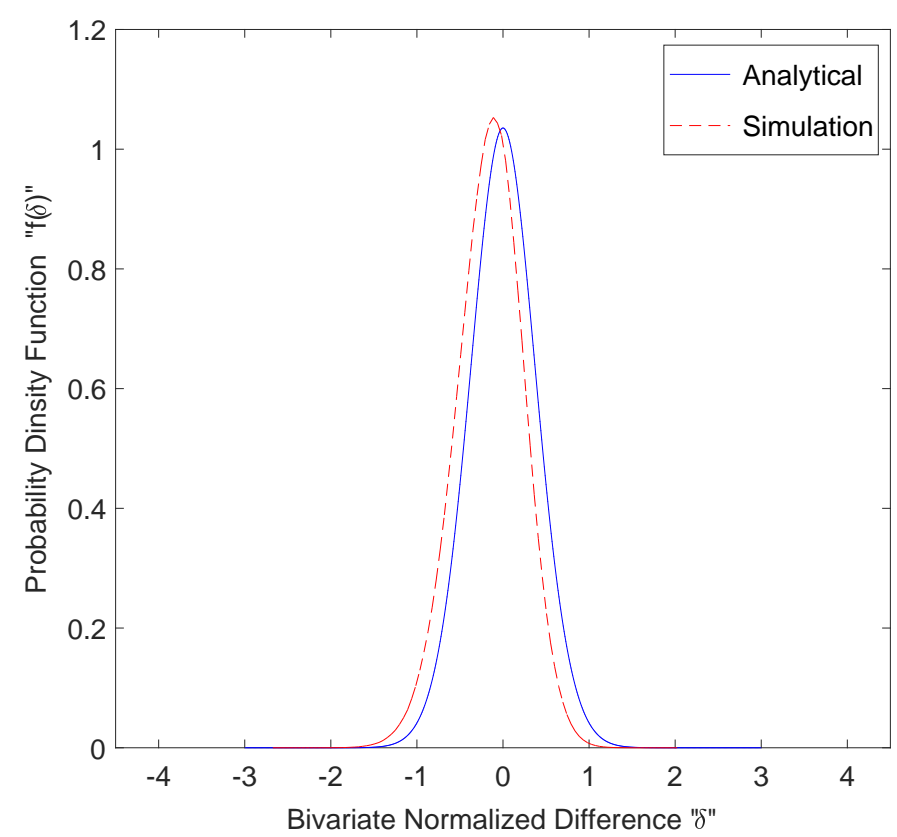

Figure 7.2: The PDF of the normalized difference of correlated Nakagami- $m$ bivariate, analytical versus simulation for $m=1$ and $\Omega=1$ and $\rho=0.3$.

It's worthy to mention that all infinite series in (7.37) converge rapidly as shown in Table 7.1 and the numerical integration can be implemented easily using commercial software.

\subsection{Outage Probability}

Outage probability $\left(P_{\text {out }}\right)$ is a key performance metric employed for testing the performance diversity techniques over fading channels. Typically, $P_{\text {out }}$ is defined as the probability of the output SNR $(\gamma)$ falls below a certain threshold, $\gamma_{t h}$ given as

$$
P_{\text {out }}=P\left(\gamma<\gamma_{t h}\right)=\int_{0}^{\gamma_{t h}} f_{\gamma}(\gamma) d \gamma
$$


Equivalently, it represents the CDF of the decorrelator evaluated at $\gamma=\gamma_{t h}$. In the following section, we will employ the outage probability as a performance metric to examine the proposed decorrelator in combating correlation among diversity branches.

\subsection{Numerical Analysis}

In this section, numerical analysis is performed to evaluate the derived expressions. First, the derived PDF expression are tested by comparing them with detailed simulation results. Then, using the outage probability as a performance metric, the effectiveness of the proposed decorrelator in combating the correlation among SC diversity branches is evaluated.

In order to compare between the analytical and simulated PDF of the difference of identical and correlated Nakagami- $m$ bivariate, Figure 7.2 is produced for $m=1, \Omega=1$ and $\rho=0.3$. In this figure, analytical and simulation are in good agreement, however, there is a slight difference between the two graphs. This can be attributed to a few reasons such as infinite series approximation, employed MATLAB simulation code for generating correlated Nakagami- $m$ random variables and PDF graph estimation. However, the graphs shapes are perfectly similar, indicating the validity of the derived expressions in describing the PDF. 


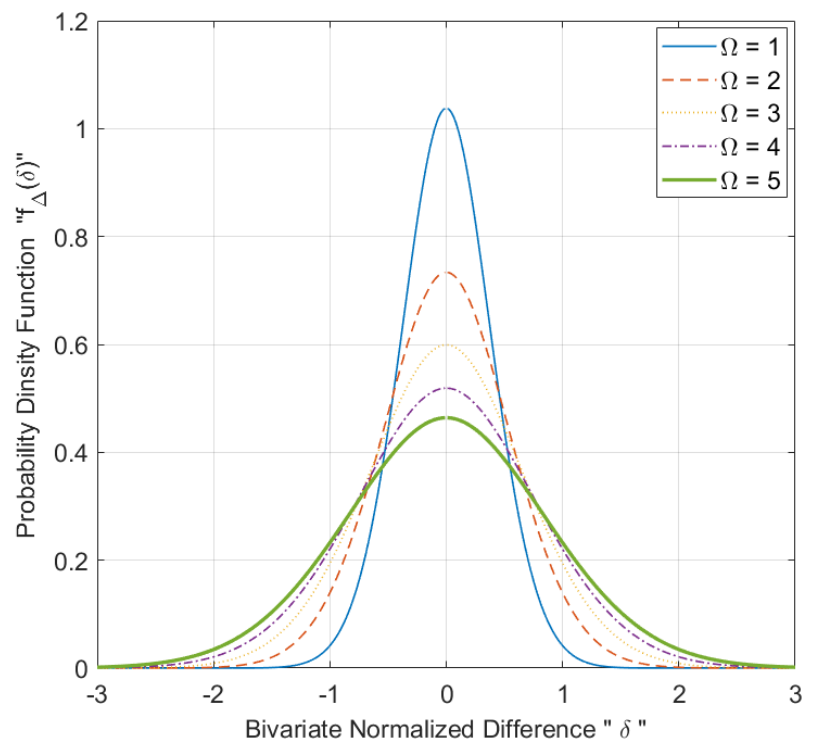

(a) $\rho=0.3$

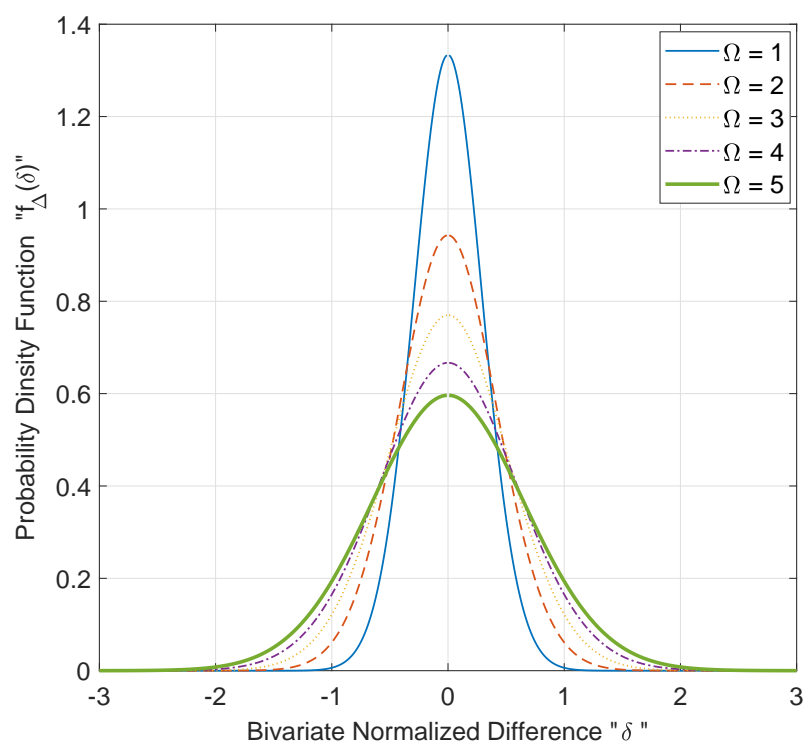

(b) $\rho=0.6$

Figure 7.3: The PDF of normalized difference of correlated Nakagami- $m$ bivariate for $m=1, \rho=0.3,0.6$ and $\Omega \in(1-5)$. 
To gain more insight on the behavior of the derived PDF expression, different fading and correlation environments are considered next. For $\rho=0.3$ and 0.6, Figures 7.3 and 7.4 are generated for $\Omega \in[1-5]$ and $m=1$, and for $\Omega=1$ and $m \in[1-4]$, respectively. These two figures reveal couple of interesting behavior.

First observation is that the produced PDF has a symmetrical bell shape, thus the PDF can be approximated to a normal distribution curve with a mean, $\mu$ and a variance, $\psi^{2}$. Another interesting observation from Figures 7.3 and 7.4 is that this mean is constant $(\mu=0)$ and independent of $m, \Omega$ and $\rho$ values. However, these values affect the variance and the scale of the graph. The same observations are seen clearly for i.i.d. bivariate. Also excellent agreement between the analytical and simulation results is achieved as shown in Figure 7.5. Therefore, we say that the PDF of the difference of identical Nakagami bivariate $(\delta)$ could be represented by a semi-standard normal distribution $\sim N\left(0, \psi^{2}\right)$ given as

$$
f(\delta)=\frac{1}{\sqrt{2 \pi \psi^{2}}} \exp \left(-\frac{1}{2 \psi^{2}} \delta^{2}\right)
$$

with zero mean and variance $\psi^{2}$ that depends on $m, \Omega$ and $\rho$.

Secondly, Figure 7.6 clearly shows an improvement on the outage probability $P_{\text {out }}$ with the decorrelated SC receiver by double compared to its value in conventional (correlated) SC receiver. This is especially obvious in the low SNR regime. Furthermore, in low SNR regime and for constant correlation values among the fading branches, we observe that as $m$ increases (fading decreases), the decorrelator performs better. 


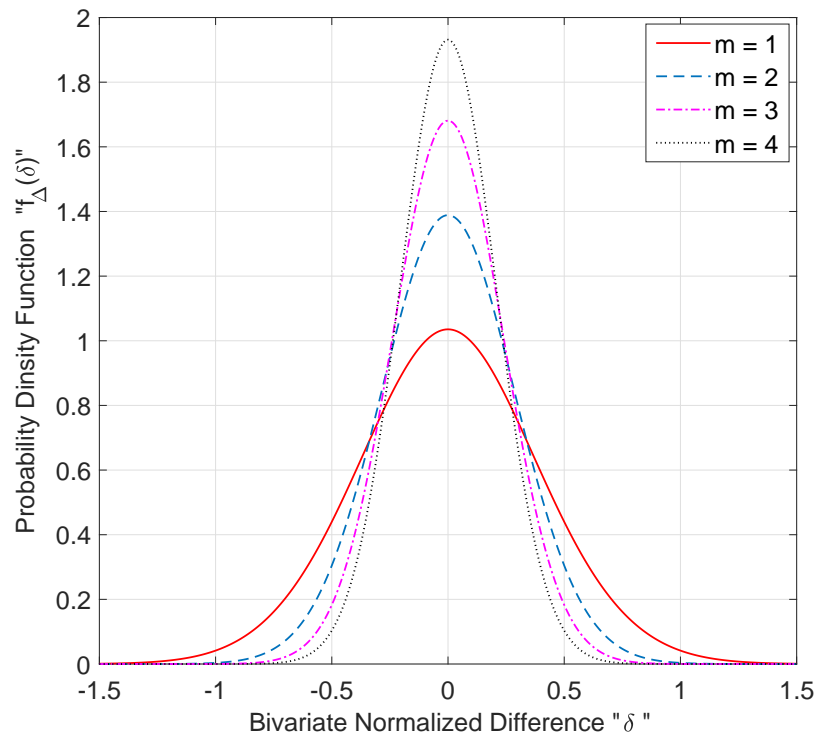

(a) $\rho=0.3$

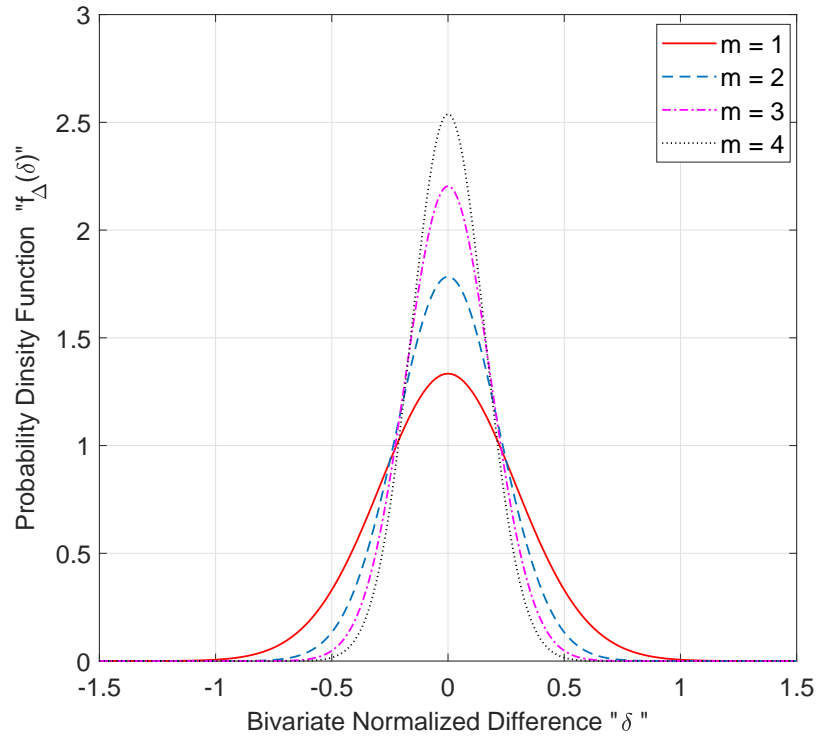

(b) $\rho=0.6$

Figure 7.4: The PDF of normalized difference of correlated Nakagami- $m$ bivariate for $\Omega=1, \rho=0.3,0.6$ and $m \in(1-4)$. 


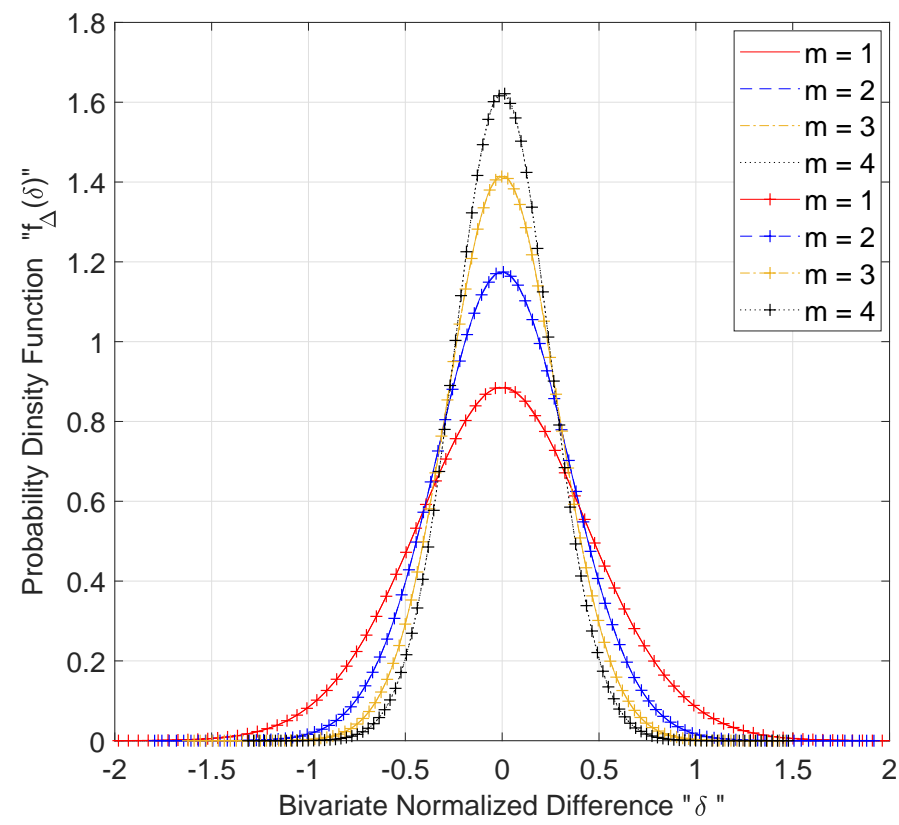

Figure 7.5: The PDF of the normalized difference of i.i.d. Nakagami- $m$ bivariate, analytical (flat) versus simulation (with marker) for $m \in(1-4)$ and $\Omega=1$. 


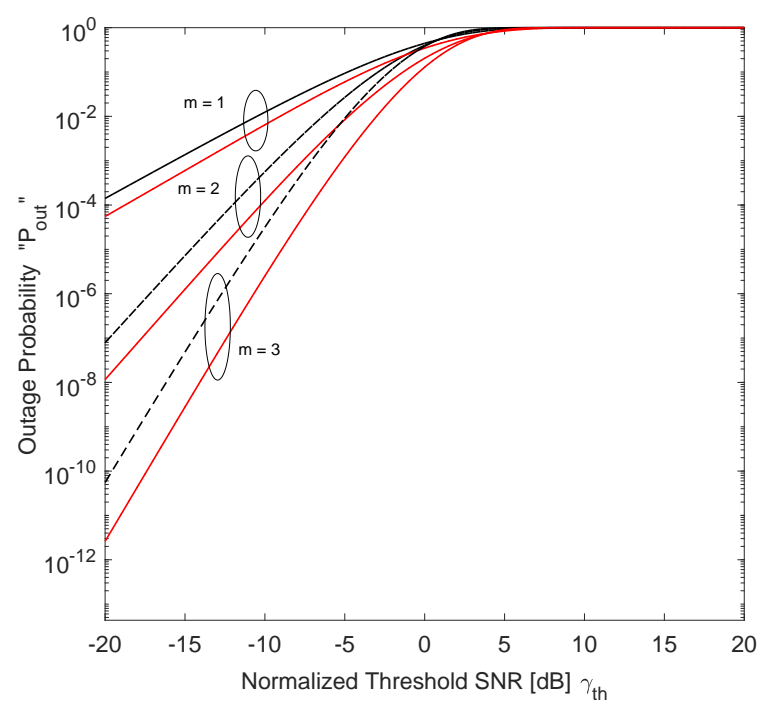

(a) $\rho=0.3$

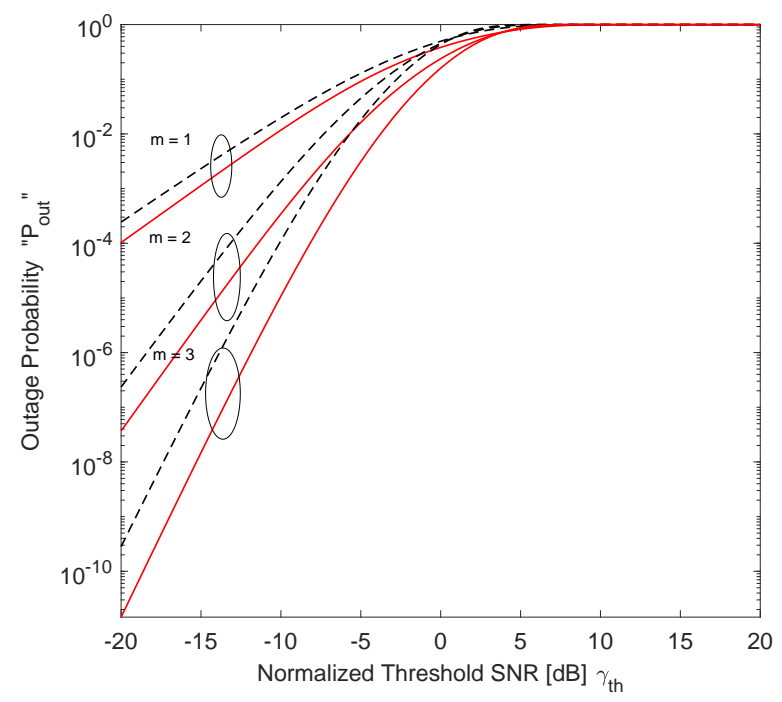

(b) $\rho=0.6$

Figure 7.6: Outage probability comparison between correlated (dashed) and decorrelated (solid) SC diversity receiver in Nakagami- $m$ channel for $\bar{\gamma}=0 \mathrm{~dB}$. 


\section{Chapter 8}

\section{Comparison of Different Schemes}

In this chapter, we perform two levels of performance comparison, namely dual and comprehensive comparison in order to study the impact of multipath fading and correlation among antenna branches on the detection probability. To this end, we use the derived closed-form expressions in previous chapters to produce the CROC for MRC, EGC, SC and SSC diversity combining techniques in Nakagami- $m$ fading channel. Detection performance for both constant and exponential correlation models are studied employing different $L$ number of diversity branches. We follow same procedures as we did in Sec. 3.4 to calculate the corresponding threshold for the assumed detection parameters to produce corresponding CROC graphs.

\subsection{MRC/EGC Comparison}

In Figure 8.1, we compare between MRC and EGC diversity techniques and their performance in combating the correlation. Results in this figure show clearly that 


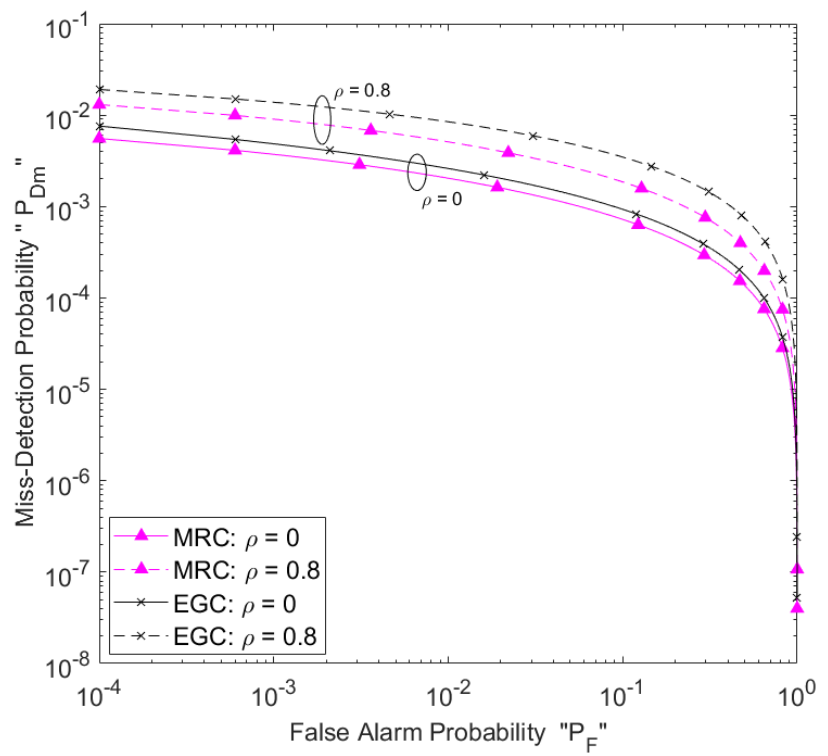

(a) $m=1$ (Rayleigh)

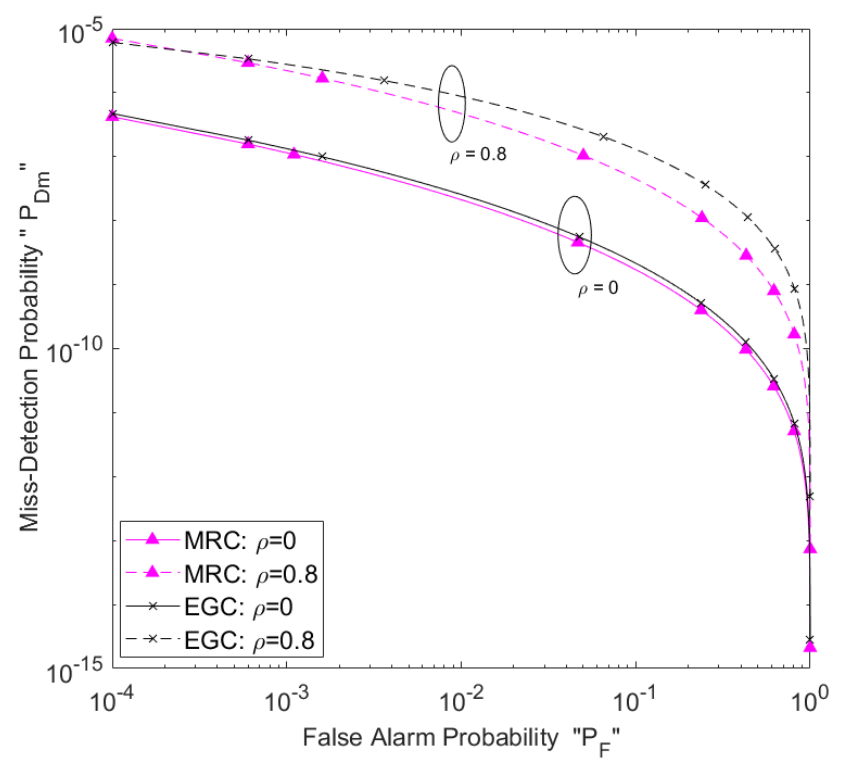

(b) $m=4$

Figure 8.1: Comparison of correlated Nakagami- $m$ branches with both MRC and EGC diversity techniques for $\bar{\gamma}=20 \mathrm{~dB}, L=2$ with $\rho=0$ (solid) and 0.8 (dashed). 
MRC is the optimal diversity combining technique compared to the suboptimal EGC technique. This is a well proven fact in the literature. This fact is more pronounced for high fading and high correlation values among diversity branches. However, a small deviation from this rule can be seen in case a good channel $(m=4)$ as shown in Figure 8.1b where their performance becomes comparable. Furthermore, for low values of probability of false alarm $P_{F}$, high correlation $(\rho=0.8)$, the EGC and MRC curves almost overlap. Therefore, at low fading, low $P_{F}$ and highly correlated environments, EGC which is a simpler scheme performs as good as MRC which is a more complex scheme.

\subsection{SC/SSC Comparison}

For easy and better comparison between the two investigated techniques (namely SC and SSC) and their performance in combating the correlation, we produce Figure 8.2. As before, one can notice the correlation impact on the detection probability with the increment in correlation between fading branches for SSC and how this impact is compensated for, due to the good fading, with almost same results as we noticed in SC. Furthermore, results in Figure 8.2 show that SC outperforms SSC. This is a well proven fact in the literature. In fact, performance difference is more pronounced for uncorrelated $(\rho=0)$ and high $m$ values. However, we may notice that as the correlation increases between the branches, the performance of both SC and SSC becomes more comparable especially for high $m$ values.

Another interesting behavior could be observed in Figure 8.3 for both SC and SSC diversity techniques. In fact, as $m$ increases (equivalently, fading decreases), less 


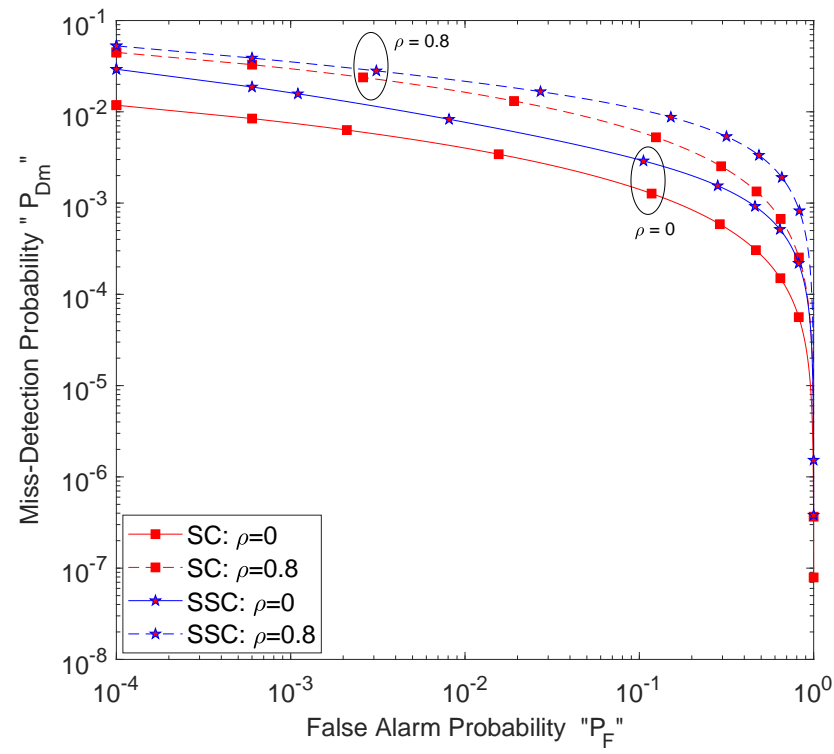

(a) $m=1$ (Rayleigh)

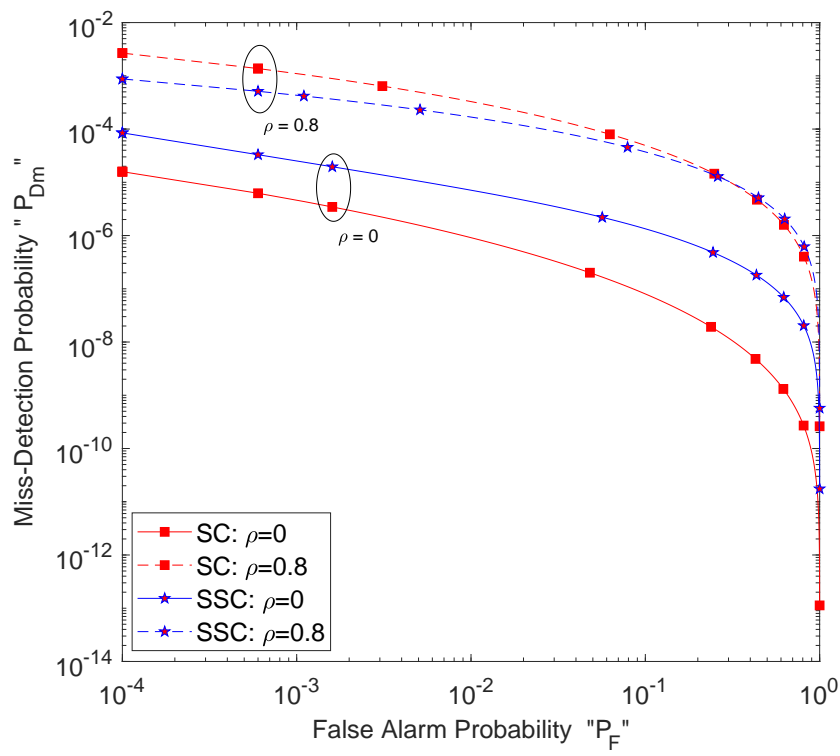

(b) $m=4$

Figure 8.2: SC and SSC dual correlated Nakagami- $m$ branches comparison with $\bar{\gamma}=20$ $\mathrm{dB}$ for $\rho=0$ (solid) and 0.8 (dashed). 
significant deterioration in detection probability is observed due to correlation. In other words, the difference between the values of $P_{D}$ is getting smaller corresponding to different correlation values between the branches which is equivalent to the loss in the diversity gain.

To gain better insight about this behavior, let us discuss it with more details. Figure 8.3a shows clearly this interesting behavior. As an example, the curve for $m=1$ in Figure 8.3a has an average high positive slope. Consequently, the detection probability degrades rapidly as correlation among fading branches increases. As $m$ increases, corresponding curves get flattened (slope decreases). Consequently, detection probability degrades slowly as correlation among fading branches increases. This can be attributed to already high detection probability $P_{D}$ values due to low fading. On the other hand, for small $m$-values (deep fading), correlation significantly deteriorates the detection probability which is already poor. Similar above behavior could be observed in case SSC as in Figure 8.3b. Therefore, we can conclude that for deep fading environment, correlation must be considered for accurately modeling cognitive radio detection, while for low fading environment (large $m$-values), the correlation effect may be ignored. Thus, reducing the complexity of calculations.

Furthermore, our investigation reveals that at low fading and highly correlated environments, SSC which is simpler scheme performs as good as SC which is a more complex scheme. 


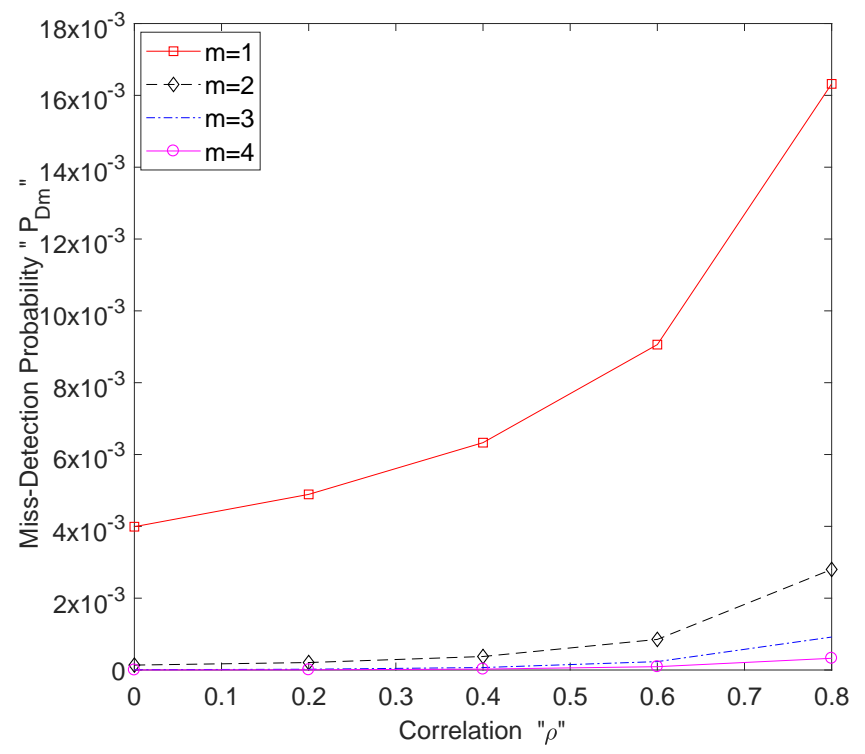

(a) $\mathrm{SC}$

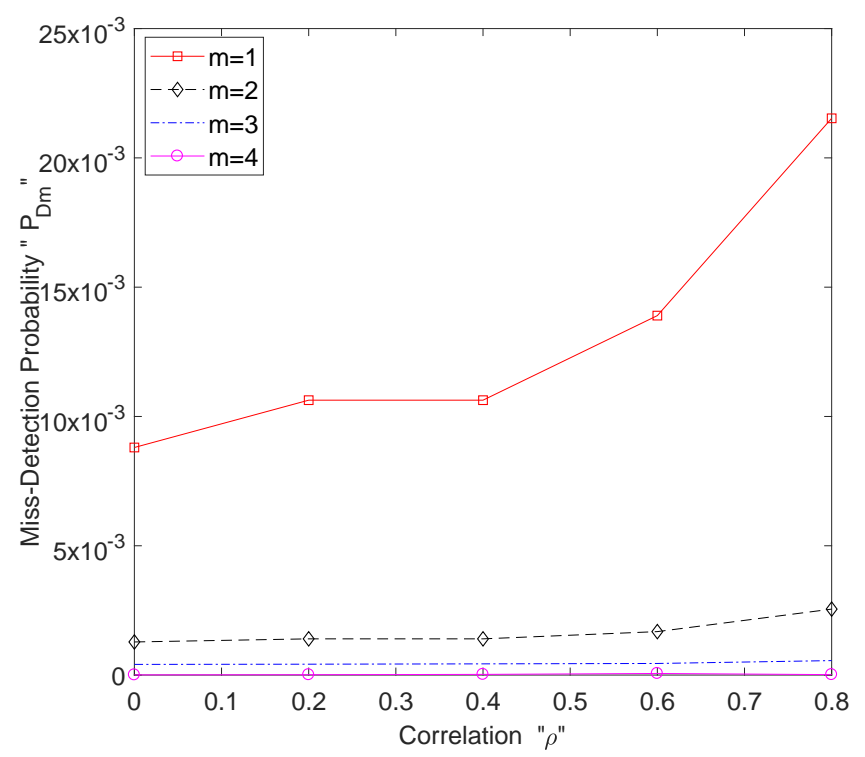

(b) $\mathrm{SSC}$

Figure 8.3: Probability of miss detection versus correlation with $\bar{\gamma}=20 \mathrm{~dB}$ and different fading severity for SC and SSC. 


\subsection{Comprehensive Comparison}

For easy and better comparison among different diversity techniques and their performance in combating the correlation, we produce Figure 8.4 and Figure 8.5. Results in above figures show that MRC is the optimal diversity combining technique compared to EGC, SC and SSC. This is a well proven fact in the literature. However, some irregularity for this rule can be seen in case of SC and SSC with $m=3$ and 4 as in Figure 8.5 (a) and (b) where SSC curve for $(\rho=0.8)$ is shifted slightly below it's counterpart for SC which can be attributed to numerically calculated optimum threshold value (which may not be very accurate).

Furthermore, as $m$ increases, less significant deterioration due to correlation in $P_{D}$ is observed. In other words, the difference between corresponding correlated and uncorrelated graphs which is equivalent to the loss in the diversity gain is getting smaller. This can be attributed to already high $P_{D}$ values due to low fading. On the other hand, for small $m$-values, correlation significantly deteriorates $P_{D}$ which is already poor. Consequently, an increased in the interference rate between the primary user and secondary user is observed by three times its rate when independent fading branches is assumed. Therefore, we can conclude, for deep fading environment, correlation must be considered for accurately modeling cognitive radio detection, while for low fading environment, the correlation effect may be ignored. 


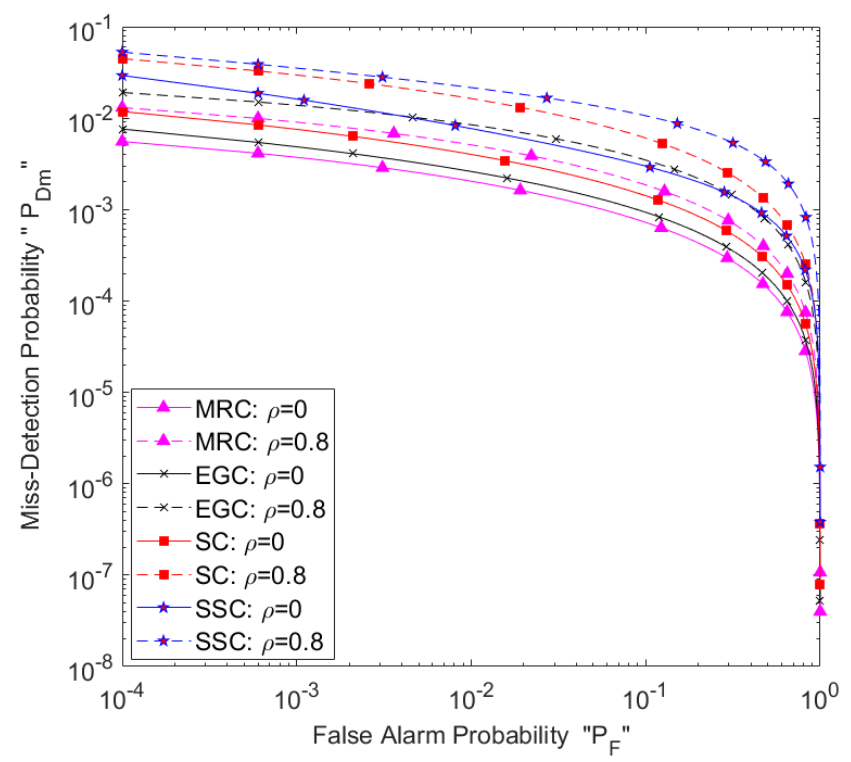

(a) $m=1$ (Rayleigh)

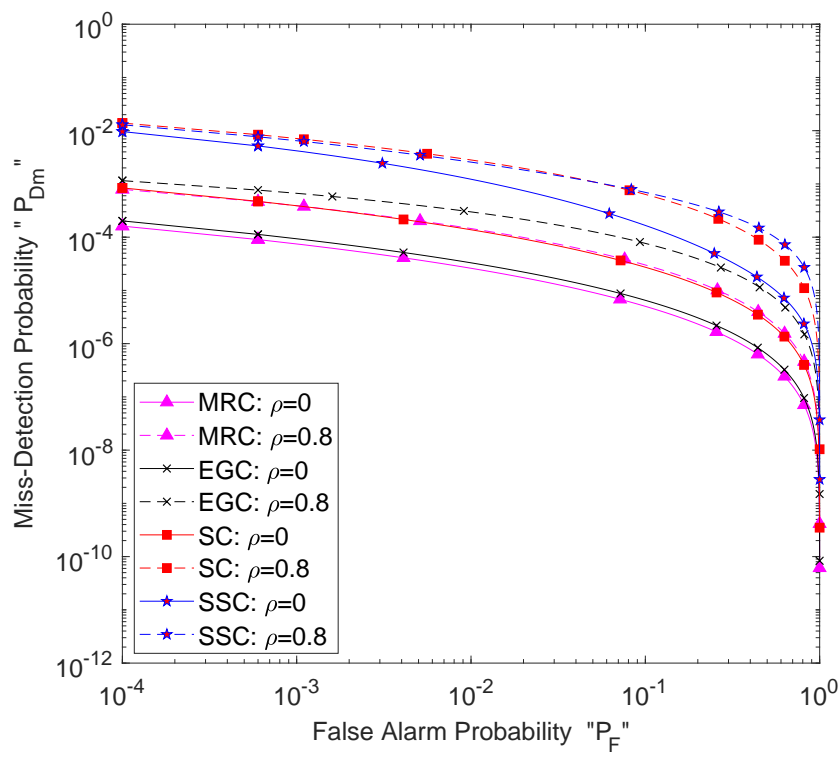

(b) $m=2$

Figure 8.4: MRC, EGC, SC and SSC correlated Nakagami- $m$ branches comparison for $L=2, \bar{\gamma}=20 \mathrm{~dB}$ and $\rho=0$ (solid) and 0.8 (dashed). 


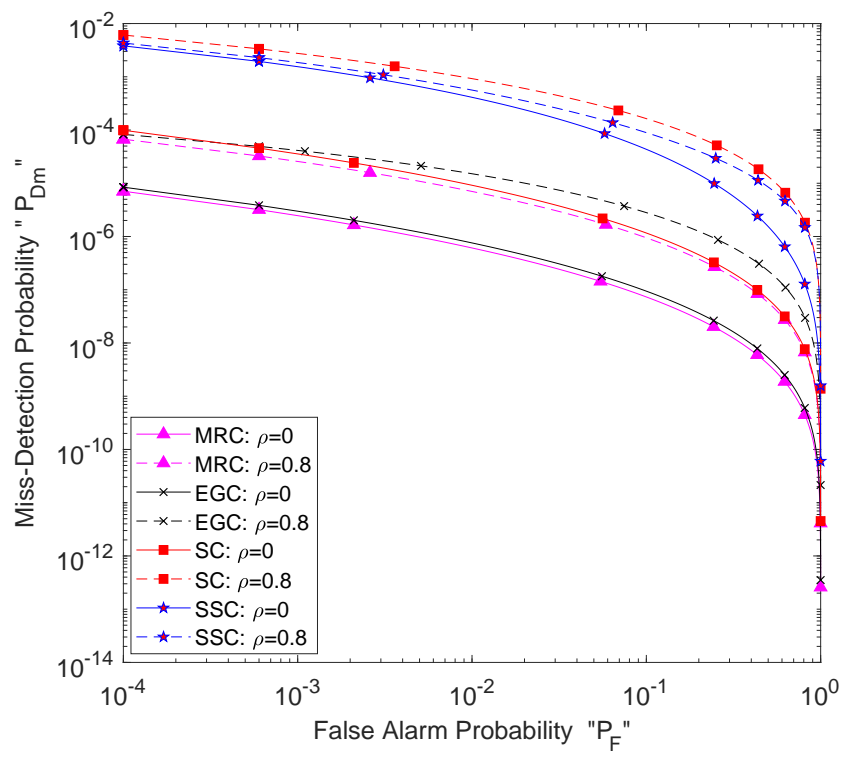

(a) $m=3$.

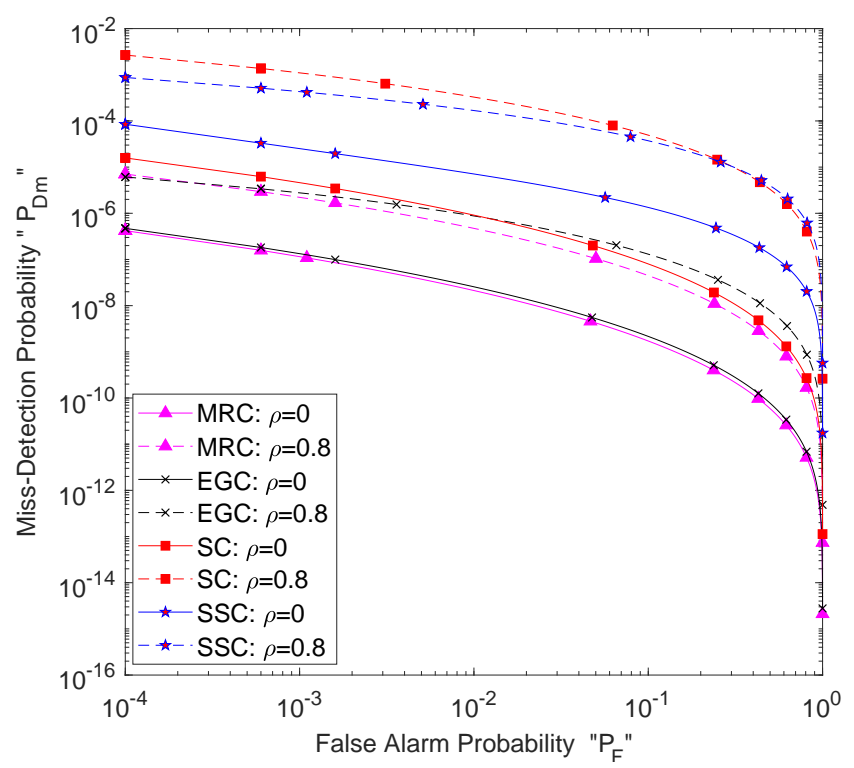

(b) $m=4$.

Figure 8.5: MRC, EGC, SC, and SSC correlated Nakagami- $m$ branches comparison for $L=2, \quad \bar{\gamma}=20 \mathrm{~dB}$ and $\rho=0$ (solid) and 0.8 (dashed). 


\subsection{Decorrelator Performance}

In the previous chapter, we verified our proposed decorrelated SC receiver. We showed in Figures 7.3 and 7.4 that the PDF of the difference of identical and correlated Nakagami- $m$ bivariate has a symmetrical bell shape. Thus, this PDF can be represented by a normal distribution with constant zero mean and variance depends on $m, \rho$ and $\Omega$. Furthermore, Figure 7.6 showed clearly the enhancement in $P_{\text {out }}$ for different correlation and normalized threshold SNR. Investigations showed also that in low SNR regime and for constant correlation values and as fading decreases, the decorrelator performs better. 


\section{Chapter 9}

\section{Conclusion and Future Work}

\subsection{Conclusion}

This dissertation investigated some important challenges in CRNSS over multipath fading channels. Most previous works assumed independent fading branches, where this invalid assumption does not reflect the real wireless communication scenario, especially with the increasingly closely spaced antennas in small mobile units. Moreover, maximal theoretical diversity gain is not attainable. Therefore and towards maximizing the dynamic spectrum sensing accuracy, maximizing secondary user throughput and reducing interference with the primary user signal, we shed light on two important relevant issues. The work of this dissertation has two aspects; multipath fading and correlation analysis; and decorrelation-based receiver solution.

Firstly, in the analysis aspect, we considered multipath fading and correlation among diversity channels, namely, n.i.i.d. branches. We implemented a unified approach 
of performance analysis for CRNSS among multiple paths in Nakagami- $m$ fading channels with arbitrary, constant and exponential correlation environment employing energy detection technique. We investigated the detection problem with MRC, EGC, SC and SSC diversity techniques for $L \geq 2$ fading branches. We derived closed-form expressions for the average detection probability for each sensing scenario with simpler and more general alternative expressions. Furthermore, we derived an expression to evaluate the optimal threshold for SSC diversity technique.

In order to gain more insight on the performance of each investigated diversity combining technique, we performed triple-levels of analysis; individual, dual and comprehensive performance analysis. We did an individual performance analysis for each employed diversity so as to evaluate and verify our derived expressions. Consequently to prove the invalid assumption of independent fading branches in sensing scenario adopted widely by the previous works in literature. As a second level, we did a performance comparison between closely similar characteristics diversity techniques, MRC/EGC and SC/SSC. As a comprehensive comparison, we did a thorough comparison between all four employed diversity techniques. Our investigations reveal the followings interesting results.

Deep fading deteriorates spectrum sensing performance results in low probability of detection values. Also, correlation among fading branches deteriorates sensing performance, especially in high fading environments. Therefore, an increased in the interference rate between the primary user and secondary user has been observed by three times its rate when independent fading branches is assumed. Therefore, in deep correlation and high fading scenarios, correlation cannot be ignored for accurate spectrum sensing. Employing diversity techniques helps mitigate both these adverse 
impacts. As fading decreases (good channel), correlation impact, relatively increases, however, sensing improves since the detection probability is already high due to good channel. In other word, the effect rate of channel improvement is higher than the correlation adverse impact, results in improved detection probability. Consequently, ignoring correlation in good channels is justifiable in order to increase the number of receiving antennas and to reduce computations complexity. This interesting results applied to all four diversity technique investigated in this thesis.

Regarding correlation model, our results investigating MRC with constant correlation model revealed better performance over exponential correlation model. In other word, MRC constant correlation model is clearly less affected by low correlation and also, to some extent, by high correlation environment compared with exponential correlation model. Consequently, results in relatively higher throughput compared with its counterpart exponential model.

Dual comparison of the performance analysis for MRC versus EGC confirmed, as well-known in literature, the superiority of MRC over EGC, especially in high fading and high correlation values among diversity branches. However, at low fading, low $P_{F}$ and highly correlated environments, EGC which is simpler scheme performs as good as MRC which is a more complex scheme. Similarly, SC outperforms SSC as it is well proven fact in the literature, too. However, our investigation revealed that at low fading and highly correlated environments, SSC which is simpler scheme performs as good as SC which is a more complex scheme. Comprehensive comparison revealed that MRC is the optimal diversity technique, then EGC, SC and SSC, respectively. However, some irregularity could be observed in the behavior of SSC due to numerically calculated optimum threshold value (which may not be very accurate) as we pointed 
out earlier.

Secondly, in the solution aspect and towards combatting the correlation impact on wireless systems, we have proposed a decorrelated Nakagami-m SC receiver with dual branches. For such decorrelator, the necessary statistics including the PDF of the normalized difference of identical and correlated Nakagami- $m$ bivariate have been derived. Numerical analysis showed some interesting results. The PDF's of the correlated bivariate could be represented by a semi-standard normal distribution with constant zero mean and a variance that depends on bivariate's parameters. This observation might be helpful in constructing the decorrelators in order to eliminate detailed derivation process. The analysis showed also improvement in outage probability by double due to the decorrelation process, especially in low SNR regime. Furthermore, our derived statistics can be used to solve the problem of self-interference in multicarrier systems as well.

\subsection{Future Work}

This thesis contributed in narrowing the gap in CRNSS challenges by addressing multipath fading and correlation issues aiming high accuracy and more protection for primary users against interference. However, as a future work, still more investigations are necessary to include other diversity techniques, involve more diversity branches and to consider different fading distributions. More importantly, is addressing the correlation issue especially with the advance in wireless communications and the current trend to use massive MIMO. As we mentioned and proved in this thesis, decorrelation is among known techniques used to mitigate or cancel the correlation 
impact among antennas completely. As another future work, we suggest to generalize employing this technique by considering MIMO systems. However, this task will be challenging since it needs using KLT technique which necessities CSI knowledge in order to diagonalize the covariance matrix. 


\section{Bibliography}

[1] E. FCC, "Docket No 03-222 Notice of proposed rule making and order," 2003.

[2] M. A. McHenry, P. A. Tenhula, D. McCloskey, D. A. Roberson, and C. S. Hood, "Chicago spectrum occupancy measurements \& analysis and a long-term studies proposal," in Proceedings of the first international workshop on Technology and policy for accessing spectrum. ACM, 2006, p. 1.

[3] I. F. Akyildiz, W.-Y. Lee, M. C. Vuran, and S. Mohanty, "A Survey on Spectrum Management in Cognitive Radio Networks," IEEE Communications magazine, vol. 46 , no. $4,2008$.

[4] K. G. Shin, H. Kim, A. W. Min, and A. Kumar, "Cognitive Radios for Dynamic Spectrum Sccess: From Concept to Reality," IEEE Wireless Communications, vol. 17 , no. 6,2010 .

[5] M. T. Masonta, M. Mzyece, and N. Ntlatlapa, "Spectrum Decision in Cognitive Radio Networks: A Survey," IEEE Communications Surveys Tutorials, vol. 15, no. 3, pp. 1088-1107, Third 2013.

[6] Z. Chkirbene and N. Hamdi, "A Survey on Spectrum Management in Cognitive Radio Networks," International Journal of Wireless and Mobile Computing, vol. 8, 
no. 2, pp. 153-165, 2015.

[7] J. Mitola, "Cognitive Radio for Flexible Mobile Multimedia Communication." in Mobile Multimedia Commun., 1999. (MoMuC '99) 1999 IEEE Int. Workshop on, 1999, pp. 3-10.

[8] S. Haykin, "Cognitive Radio: Brain-Empowered Wireless Communication." Selected Areas in Commun., IEEE Journal on, vol. 23, no. 2, pp. 201-220, Feb. 2005.

[9] C. R. Stevenson, G. Chouinard, Z. Lei, W. Hu, S. J. Shellhammer, and W. Caldwell, "IEEE 802.22: The First Cognitive Radio Wireless Regional Area Network Standard," IEEE commun. magazine, vol. 47, no. 1, pp. 130-138, 2009.

[10] Y. Zeng, C. Koh, and Y.-C. Liang, "Maximum Eigenvalue Detection: Theory and Application," in IEEE Int. Conf. on Commun., 2008. ICC' 08., May 2008, pp. $4160-4164$.

[11] A. Goldsmith, Wireless communications. Cambridge university press, 2005.

[12] A. Ghasemi and E. S. Sousa, "Spectrum Sensing in Cognitive Radio Networks: Requirements, Challenges and Design Trade-offs," IEEE Commun. magazine, vol. 46 , no. 4,2008 .

[13] L. Cardoso, M. Debbah, P. Bianchi, and J. Najim, "Cooperative Spectrum Sensing Using Random Matrix Theory," in Wireless Pervasive Computing, 2008. ISWPC 2008. 3rd Int. Symposium on, May 2008, pp. 334-338.

[14] J. Salz and J. H. Winters, "Effect of Fading Correlation on Adaptive Arrays in Digital Wireless Communications," in Commun., 1993. ICC'93 Geneva. Technical 
Program, Conf. Record, IEEE Int. Conf. on, vol. 3. IEEE, 1993, pp. 1768-1774.

[15] — - "Effect of Fading Correlation on Adaptive Arrays in Digital Mobile Radio," IEEE trans. on Vehicular Technology, vol. 43, no. 4, pp. 1049-1057, 1994.

[16] P. Lombardo, G. Fedele, and M. M. Rao, "MRC Performance for Binary Signals in Nakagami Fading with General Branch Correlation," IEEE Trans. on Commun., vol. 47, no. 1, pp. 44-52, 1999.

[17] U. Madhow, Fundamentals of Digital Communication. Cambridge University Press, 2008.

[18] M.-S. Alouini and M. K. Simon, "Multichannel Reception of Digital signals over Correlated Nakagami Fading Channels," in Proc. of the Annual Allerton Conf. on Commun. Control and Computing, vol. 36. Citeseer, 1998, pp. 146-155.

[19] M. K. Simon and M.-S. Alouini, "Digital Communication over Fading Channels," JOHN WILEY and SONS, INC,2005, 2005,ISBN 0-471-20069-7.

[20] A. Leon-Garcia, "Probability, Statistics, and Random Processes for Electrical Engineering”. Pearson/Prentice Hall 3rd ed. Upper Saddle River, NJ, 2008.

[21] H. Urkowitz, "Energy Detection of Unknown Deterministic Signals," Proceedings of the IEEE, vol. 55, no. 4, pp. 523-531, Apr. 1967.

[22] V. Kostylev, "Energy Detection of a Signal with Random Amplitude," in IEEE Int. Conf. on Commun., ICC 2002., vol. 3, Apr-May 2002, pp. 1606-1610 vol.3.

[23] F. Digham, M.-S. Alouini, and M. K. Simon, "On the Energy Detection of Unknown Signals over Fading Channels," in Commun., 2003. ICC '03. IEEE Int. Conf., vol. 5, May 2003, pp. 3575-3579 vol.5. 
[24] —_ "On the Energy Detection of Unknown Signals Over Fading Channels," Commun., IEEE Trans. on, vol. 55, no. 1, pp. 21-24, Jan. 2007.

[25] A. Pandharipande and J.-P. Linnartz, "Performance Analysis of Primary User Detection in a Multiple Antenna Cognitive Radio," in IEEE Int. Conf. on Commun., 200\%. ICC '0\%., Jun. 2007, pp. 6482-6486.

[26] V. Kuppusamy and R. Mahapatra, "Primary user detection in OFDM based MIMO Cognitive Radio," in 2008 3rd Int. Conf. on Cognitive Radio Oriented Wireless Networks and Commun. (CrownCom 2008), May 2008, pp. 1-5.

[27] C. H. Hwang, G. L. Lai, and S. C. Chen, "Spectrum sensing in wideband ofdm cognitive radios," IEEE Trans. on Signal Processing, vol. 58, no. 2, pp. 709-719, Feb 2010.

[28] J. H. Lee, J. H. Baek, and S. H. Hwang, "Collaborative Spectrum Sensing using Energy Detector in Multiple Antenna System," in 2008 10th Int. Conf. on Advanced Communication Technology, vol. 1, Feb. 2008, pp. 427-430.

[29] S. Kim, J. Lee, H. Wang, and D. Hong, "Sensing Performance of Energy Detector with Correlated Multiple Antennas," IEEE Signal Processing Letters, vol. 16, no. 8, pp. 671-674, 2009.

[30] S. Herath and N. Rajatheva, "Analysis of Equal Gain Combining in Energy Detection for Cognitive Radio over Nakagami Channels," in Global Telecommun. Conf., IEEE GLOBECOM 2008., Nov. 2008, pp. 1-5.

[31] S. P. Herath, N. Rajatheva, and C. Tellambura, "On the Energy Detection of Unknown Deterministic Signal Over Nakagami Channels with Selection 
Combining," in Canadian Conf. on Electrical and Computer Engineering, 2009. CCECE'09. IEEE, 2009, pp. 745-749.

[32] S. P. Herath and N. Rajatheva, "Analysis of Diversity Combining in Energy Detection for Cognitive Radio over Nakagami Channels," in IEEE Commun. Society, 2009. IEEE ICC 2009 proc.. IEEE. IEEE, 2009, pp. 1-5.

[33] S. P. Herath, N. Rajatheva, and C. Tellambura, "Unified approach for Energy Detection of Unknown Deterministic Signal in Cognitive Radio Over Fading Channels," in IEEE Int. Conf. on Commun. Workshops, 2009. ICC Workshops 2009. IEEE, 2009, pp. 1-5.

[34] S. Herath, N. Rajatheva, and C. Tellambura, "Energy Detection of Unknown Signals in Fading and Diversity Reception," IEEE Trans. on Commun., vol. 59, no. 9, pp. 2443-2453, Sep. 2011.

[35] V. R. S. Banjade, N. Rajatheva, and C. Tellambura, "Performance Analysis of Energy detection with Multiple Correlated Antenna Cognitive Radio in Nakagami-m Fading," IEEE Commun. Letters, vol. 16, no. 4, pp. 502-505, 2012 .

[36] Y. F. Sharkasi, D. McLernon, and M. Ghogho, "Cooperative Spectrum Sensing Over Frequency-Selective Nakagami-m Fading Channels," in Sensor Signal Processing for Defence (SSPD 2012). IET, 2012, pp. 1-5.

[37] Q. Shi, "On the Performance of Energy Detection for Spectrum Sensing in Cognitive Radio Over Nakagami-Lognormal Composite Channels," in 2013 IEEE China Summit and Int. Conf. on Signal and Information Processing, July 2013, pp. 566-569. 
[38] E. Adebola and A. Annamalai, "Unified Analysis of Energy Detectors with Diversity Reception in Generalised Fading Channels," IET Commun., vol. 8, no. 17, pp. 3095-3104, 2014.

[39] V. R. S. Banjade, C. Tellambura, and H. Jiang, "Spectrum Sensing Performance of p-Norm Detector in Random Network Interference," in 2015 IEEE Int. Conf. on Commun. (ICC), Jun. 2015, pp. 7474-7479.

[40] A. Assra, J. Yang, and B. Champagne, "An EM Approach for Cooperative Spectrum Sensing in Multiantenna CR Networks," IEEE Trans. on Vehicular Technology, vol. 65, no. 3, pp. 1229-1243, Mar. 2016.

[41] A. Assra, A. Vakili, and B. Champagne, "Iterative Joint channel and Noise Variance Estimation and Primary User Signal Detection for Cognitive Radios," in 2011 IEEE Int. Symposium on Signal Processing and Information Technology (ISSPIT), Dec. 2011, pp. 305-309.

[42] A. Assra and B. Champagne, "EM-Based Joint Estimation and Detection for Multiple Antenna Cognitive Radios," in 2012 IEEE Int. Conf. on Commun. (ICC), Jun. 2012, pp. 1482-1486.

[43] Q. Song and W. Hamouda, "A cooperative Sensing Scheme for Cognitive Networks Over Fading Channels," in 2016 Int. Wireless Commun. and Mobile Computing Conf. (IWCMC), Sep. 2016, pp. 632-637.

[44] A. A. Hammadi, O. Alhussein, P. C. Sofotasios, S. Muhaidat, M. Al-Qutayri, S. Al-Araji, G. K. Karagiannidis, and J. Liang, "Unified Analysis of Cooperative Spectrum Sensing Over Composite and Generalized Fading Channels," IEEE Trans. on Vehicular Technology, vol. 65, no. 9, pp. 6949-6961, Sep. 2016. 
[45] S. Atapattu, C. Tellambura, and H. Jiang, "A mixture Gamma Distribution to Model the SNR of Wireless Channels," IEEE trans. on wireless commun., vol. 10, no. 12, pp. 4193-4203, 2011.

[46] O. Alhussein, S. Muhaidat, J. Liang, and P. D. Yoo, "A unified Approach for Representing Wireless Channels using EM-Based Finite Mixture of Gamma Distributions," in Globecom Workshops (GC Wkshps), 2014. IEEE, 2014, pp. $1008-1013$.

[47] X. Dong and N. C. Beaulieu, "Optimal Maximal Ratio Combining with Correlated Diversity Branches," IEEE Commun. letters, vol. 6, no. 1, pp. 22-24, 2002.

[48] G. R. Tsouri, D. Wulich, and L. Goldfeld, "Enhancing Switched Diversity Systems," in Sensor Array and Multichannel Signal Processing Workshop Proceedings, 2004. IEEE, 2004, pp. 485-488.

[49] —_ "Enabling Dual Branch Switched Diversity in Compact Wireless Devices," IEEE Trans. on Consumer Electronics, vol. 54, no. 4, pp. 1545-1549, Nov. 2008.

[50] P. O. Akuon and H. Xu, "Optimal Error Analysis of Received Diversity Schemes on Arbitrarily Correlated Rayleigh Fading Channels," IET Communications, vol. 10, no. 7, pp. 854-861, 2016.

[51] S. Haghani and N. C. Beaulieu, "On Decorrelation in Dual-Branch Diversity Systems," IEEE Trans. on Commun., vol. 57, no. 7, pp. 2138-2147, July 2009.

[52] K. T. Hemachandra and N. C. Beaulieu, "Simple Expressions for the SER of Dual MRC in Correlated Nakagami-q (Hoyt) Fading," IEEE Commun. Letters, vol. 14 , no. 8, pp. 743-745, 2010. 
[53] — - "Novel Analysis for Performance Evaluation of Energy Detection of Unknown Deterministic Signals Using Dual Diversity," in Vehicular Technology Conference (VTC Fall), 2011 IEEE. IEEE, 2011, pp. 1-5.

[54] J. T. Wang, "Receive Combiner Bank for MIMO System under Multi-User Cochannel Interference," IEEE Communications Letters, vol. 16, no. 3, pp. 328-330, March 2012.

[55] — - "MIMO System With Receive Combiner Bank and Power Control for Cognitive Radio Networks," IEEE Transactions on Vehicular Technology, vol. 62, no. 8, pp. 3767-3773, Oct. 2013.

[56] — - "Decorrelation based receive transformation for mimo system under multi-user co-channel interference," IEEE Wireless Commun. Letters, vol. 3, no. 3, pp. 305-308, 2014.

[57] A. Bishnu and N. Tiwari, "MIMO System Channel Capacity with Input Transformation," in 2015 International Conference on Computer, Communication and Control (IC4), Sep. 2015, pp. 1-4.

[58] Y. Zeng and Y.-C. Liang, "Spectrum-Sensing Algorithms for Cognitive Radio based on Statistical Covariances," IEEE trans. on Vehicular Technology, vol. 58, no. 4, pp. 1804-1815, 2009.

[59] D. Bera, I. Chakrabarti, S. S. Pathak, and G. K. Karagiannidis, "Another Look in the Analysis of Cooperative Spectrum Sensing over Nakagami- $m$ Fading Channels," IEEE Trans. on Wireless Communications, vol. 16, no. 2, pp. 856-871, Feb. 2017. 
[60] I. F. Akyildiz, W.-Y. Lee, M. C. Vuran, and S. Mohanty, "NeXt Generation/Dynamic Spectrum Access/Cognitive Radio Wireless Networks: A Survey," Computer Networks, vol. 50, no. 13, pp. 2127-2159, 2006.

[61] A. Taherpour, M. Nasiri-Kenari, and S. Gazor, "Multiple Antenna Spectrum Sensing in Cognitive Radios," Wireless Commun., IEEE Trans. on, vol. 9, no. 2, pp. 814-823, Feb. 2010.

[62] W. Gardner, "Signal Interception: A Unifying Theoretical Framework for Feature Detection," Commun., IEEE Trans. on, vol. 36, no. 8, pp. 897-906, Aug. 1988.

[63] P. Sutton, K. Nolan, and L. Doyle, "Cyclostationary Signatures in Practical Cognitive Radio Applications," Selected Areas in Commun., IEEE Journal on, vol. 26, no. 1, pp. 13-24, Jan. 2008.

[64] B. Sklar, "Digital Communications: Fundamentals and Applications", ser. Prentice Halls Int. editions. Prentice-Hall, 1988. [Online]. Available: http://books.google.ca/books?id=HbW3QgAACAAJ

[65] F. Commission et al., "IEEE 802.22 Working Group on Wireless Regional Area Networks," Federal Commun. Commission, Tech. Rep., 2006, Available: http://grouper.ieee.org/groups/802/22/.

[66] V. A. Aalo, "Performance of maximal-ratio diversity systems in a correlated nakagami-fading environment," IEEE Trans. on Commun.,, vol. 43, no. 8, pp. 2360-2369, 1995.

[67] S. L. Loyka, "Channel Capacity of MIMO Architecture Using the Exponential Correlation Matrix," IEEE Commun. letters, vol. 5, no. 9, pp. 369-371, 2001. 
[68] S. Kotz and J. W. Adams, "Distribution of Sum of Identically Distributed Exponentially Correlated Gamma-Variables," The Annals of Mathematical Statistics, pp. 277-283, 1964.

[69] P. Bianchi, M. Debbah, M. Maida, and J. Najim, "Performance of Statistical Tests for Single-Source Detection Using Random Matrix Theory," Information Theory, IEEE Trans. on, vol. 57, no. 4, pp. 2400-2419, Apr. 2011.

[70] G. Turin, "On Optimal Diversity Reception, II," IRE Trans. on Commun. Systems, vol. 10, no. 1, pp. 22-31, 1962.

[71] A. Annamalai, O. Olabiyi, S. Alam, O. Odejide, and D. Vaman, "Unified Analysis of Energy Detection of Unknown Signals Over Generalized Fading Channels," in Wireless Commun. and Mobile Computing Conf. (IWCMC), 2011 7th Int. IEEE, 2011, pp. 636-641.

[72] V. A. Thomas, S. Kumar, S. Kalyani, M. El-Hajjar, K. Giridhar, and L. Hanzo, "Error Vector Magnitude Analysis of Fading SIMO Channels Relying on MRC Reception," IEEE Trans. on Commun., vol. 64, no. 4, pp. 1786-1797, Apr 2016.

[73] K. Cho, J. Lee, and C. G. Kang, "Stochastic Geometry-Based Coverage and Rate Analysis Under Nakagami \& Log-normal Composite Fading Channel for Downlink Cellular Networks," IEEE Communications Letters, 2017.

[74] M. Nakagami, "The m-Distribution-A General Formula of Intensity Distribution of Rapid Fading," Statistical Method of Radio Propagation, 1960.

[75] M. Abramowitz and I. A. Stegun, "Handbook of Mathematical Functions: with Formulas, Graphs, and Mathematical Tables". Courier Dover Publications, 1972, no. 55 . 
[76] M. K. Simon and M.-S. Alouini, "A Unified Approach to the Performance Pnalysis of Digital Communication Over Generalized Fading Channels," Proceedings of the IEEE, vol. 86, no. 9, pp. 1860-1877, Sep 1998.

[77] I. Gradshteyn and I. Ryzhik, " Table of Integrals, Series and Products," Alan Jeffrey and Daniel Zwillinger (eds.), Seventh edition (Feb 2007), p. 885, 2007.

[78] S. Al-Juboori and X. Fernando, "Unified Approach for Performance Analysis of Cognitive Radio Spectrum Sensing Over correlated Multipath Fading Channels," in WoWMoM, 2015 IEEE 16th Int. Symposium. IEEE, Boston, USA, 2015, pp. $1-6$.

[79] — _ "Multi-Antenna Spectrum Sensing over Correlated Nakagami-m Channels with MRC and EGC Diversity Reception," in IEEE trans. on Vehicular Technology, Under second round of revision, May $201 \%$.

[80] C. C. Tan and N. C. Beaulieu, "Infinite Series Representations of the Bivariate Rayleigh and Nakagami-m Distributions," Commun., IEEE Trans. on, vol. 45, no. 10, pp. 1159-1161, 1997.

[81] A. H. Nuttall, "Some Integrals Involving the Q-Function," DTIC Document, Tech. Rep., 1974.

[82] P. C. Sofotasios, M. Valkama, T. A. Tsiftsis, Y. A. Brychkov, S. Freear, and G. K. Karagiannidis, "Analytic Solutions to a Marcum Q-function-Based Integral and Application in Energy Detection of Unknown Signals Over Multipath Fading Channels," in 9th Int. Conf. on Cognitive Radio Oriented Wireless Networks and Commun. (CROWNCOM), 2014. IEEE, 2014, pp. 260-265. 
[83] C.-D. Iskander and P. Mathiopoulos, "Performance of M-QAM with Coherent Equal-Gain Combining in Correlated Nakagami-m Fading," Electronics letters, vol. 39, no. 1, pp. 141-142, 2003.

[84] G. L. Stuber, Principles of Mobile Communication, ser. 2002 Kluwer Academic Publishers.

[85] G. Fedele, L. Izzo, and M. Tanda, "Dual Diversity Reception of M-ary DPSK Signals Over Nakagami Fading Channels," in Personal, Indoor and Mobile Radio Commun., 1995. PIMRC'95. Wireless: Merging onto the Information Superhighway., Sixth IEEE Int. Symposium on, vol. 3, Sep. 1995, pp. 1195-1201.

[86] S. Al-Juboori and X. Fernando, "Correlated Multichannel Spectrum Sensing Cognitive Radio System with Selection Combining," in IEEE Global Commun. Conf. (GLOBECOM), 2016 IEEE. IEEE, Washington DC, USA, 2016, pp. 1-6.

[87] S. Al-Juboori, Y. D. Fernando, Xavier, and A. Nallanathan, "Impact of Inter-Branch Correlation on Multichannel Spectrum Sensing with SC and SSC Diversity Combining," in IEEE Trans. on Vehicular Comm. and Networking, 2017 IEEE, Under Review.

[88] G. K. Karagiannidis, D. Zogas, S. Kotsopoulos et al., "An Efficient Approach to Multivariate Nakagami-m Distribution Using Green's Matrix Approximation," Wireless Commun., IEEE Trans., vol. 2, no. 5, pp. 883-889, 2003.

[89] G. K. Karagiannidis, D. A. Zogas, and S. A. Kotsopoulos, "Performance Analysis of Triple Selection Diversity Over Exponentially Correlated Nakagami-m Fading Channels," Commun., IEEE Trans., vol. 51, no. 8, pp. 1245-1248, 2003. 
[90] N. M. Temme, "Computational Aspects of Incomplete Gamma Functions with Large Complex Parameters," in Approximation and Computation: A Festschrift in Honor of Walter Gautschi. Springer, 1994, pp. 551-562.

[91] D. Yang, H. Ai, C. Kyriakakis, and C.-C. J. Kuo, "Adaptive Karhunen-Loeve Transform for Enhanced Multichannel Audio Coding," in Int. Symposium on Optical Science and Technology. Int. Society for Optics and Photonics, 2001, pp. $43-54$.

[92] Y. Chen and C. Tellambura, "Distribution Functions of Selection Combiner Output in Equally Correlated Rayleigh, Rician, and Nakagami-m Fading Channels," IEEE Trans. on Commun., vol. 52, no. 11, pp. 1948-1956, Nov 2004 .

[93] S. Al-Juboori and X. Fernando, "Decorrelation-Based Selection Combining Receiver in Nakagami-m Fading Channel," in IEEE Transactions on Communications, 2017 IEEE, Under Review.

[94] A. Ligeti, "Outage Probability in the Presence of Correlated Lognormal Useful and Interfering Components," IEEE Commun. Letters, vol. 4, no. 1, pp. 15-17, 2000.

[95] H. Holm and M.-S. Alouini, "Sum and Difference of Two Squared Correlated Nakagami Variates in Connection with the McKay Distribution," IEEE Trans. on Commun., vol. 52, no. 8, pp. 1367-1376, 2004.

[96] C. D. Iskander and P. T. Mathiopoulos, "Performance Analysis of M-PSK, DE-M-PSK and M-QAM with Dual-Branch Coherent Equal-Gain and Maximal-Ratio Combining in Correlated Nakagami-m Fading," in Electrical 
and Computer Engineering, 2003. IEEE CCECE 2003. Canadian Conf. on, vol. 3, May 2003, pp. 1683-1686 vol.3. 